\title{
Image Hierarchy in Gaussian Scale Space
}

Tomoya Sakai ${ }^{1,4}$, Masaki Narita $^{2}$, Takuto, Komazaki ${ }^{3}$, Haruhiko Nishiguchi $^{3}$, Atsushi Imiya ${ }^{1,2}$

1. Institute of Media and Information Technology, Chiba University

2. Graduate School of Advanced Integration Science, Chiba University

3. Graduate School of Science and Technology, Chiba University ${ }^{1}$

4. Graduate School of Engineering, Chiba University

Yayoi-cho 1-33, Inage-ku, 263-8522, Chiba, Japan

${ }^{1}$ Parts of this paper are based on the Master thesis of T. Komazaki and H. Nishiguch at Chiba University in the academic years 2008 and 2007, respectively. 


\begin{abstract}
We investigate the topological structure of an image and the hierarchical relationship between local and global structures provided by spatial gradients at different levels of scale in the Gaussian scale space. The gradient field curves link stationary points of an image including a local minimum at infinity, and construct the topological structure of the image. The evolution of the topological structure with respect to scale is analysed using pseudograph representation. The hierarchical relationships among the structures at different scales are expressed as trajectories of the stationary points in the scale space, which we call stationary curves. Each top-point of the local extremum curve generically has a specific gradient field curve, which we call the antidirectional figure-flow curve. The antidirectional figure-flow curve connects between the top-point and another local extremum to which the toppoint is subordinate. A point at infinity can also be connected to the toppoints of local minimum curves. These hierarchical relationships among the stationary points are expressed as a tree. This tree expresses a hierarchical structure of dominant parts. We clarify the graphical grammar for the construction of this tree in the Gaussian scale space. Furthermore, we show a combinatorial structure of singular points in the Gaussian scale space using conformal mapping from Euclidean space to the spherical surface. We define segment edges as a zero-crossing set in the Gaussian scale space using the singular points. An image in the Gaussian scale space is the convolution of the image and the Gaussian kernel. The Gaussian kernel of an appropriate variance is a typical presmoothing operator for segmentation. The variance is heuristically selected using statistics of images such as the noise distribution in images. The variance of the kernel is determined using the singular-point configuration in the Gaussian scale space, since singular points in the Gaussian scale space allow the extraction of the dominant parts of an image. This scale selection strategy derives the hierarchical structure of the segments. Unsupervised segmentation methods, however, have difficulty in distinguishing valid segments associated with the objects from invalid random segments due to noise. Showing that the number of invalid segments monotonically decreases with increasing scale, we characterise thevalid and invalid segments in the Gaussian scale space. This property allows us to identify the valid segments from coarse to fine, and to prevent under- and oversegmentation. Finally, we develop principal component analysis (PCA) of a point cloud on the basis of scale-space representation of its probability density function (PDF). We explain the geometric features of a point cloud in the Gaussian scale space, and observe reduction of dimensionality with respect to the loss of information. Furthermore, we introduce a hierarchical clustering of the point cloud, and analyse the statistical significance of the clusters and their subspaces. Moreover, we present a mathematical framework of the scale-based PCA, which derives a statistically reasonable criterion for choosing the number of components to retain, or reducing the dimensionality of point cloud. Finaly, we also develop a segmentation algorithm using configurations of singular points in the Gaussian scale space.
\end{abstract}

AMC 2010: 62H35,68T45, 68R10, 62H30, 05C21, 65F05 


\section{Introduction}

The aim of this paper is to clarify the hierarchical relationship between the local structure and global structure of an image using spatial gradients in the Gaussian scale space. The grey-scale image consists of a number of regions expressed by the luminance distribution in space. Stationary points, or critical points, are mathematically well-defined feature points of the image. Local maxima, local minima, and saddle points respectively represent bright and dark regions, and in-between segments in the space where the image resides. The adjacencies of the feature points of the image present a topological structure. Since the stationary points are the points where the gradient vanishes, gradient field curves provide the adjacencies and show the topological structure, that is, the gradient structure.

As image detail depends on the resolution or scale of the image, the gradient structure is also governed by the scale. The theory of the Gaussian scale space goes back to Iijima (Iijima, 1962, 1963, 1971, 1973, 1999; Weickert, Ishikawa, and Imiya, 1997, 1999), who firstly introduced the linear diffusion equation as the fundamental equation of figures starting from four axioms on image operations.

- Non-negative intensity of figures imaged on the retina.

- Linearity of image intensity.

- Closedness under affine transformations.

- Associative (or semigroup) property.

Later Otsu (Otsu, 1981) established a formal formalisation of the Gaussian scale-space theory employing the Lie group theory. Zhao and Iijima (Zhao and Iijima, 1985a) extended Iijima's theory to extract the hierarchical structure of images using singular points of topography of a grey-scale image in a linear scale space. In 1983, Witkin published a paper on scale-space filtering, and clarified the importance of the Gaussian-kernel filter in image processing (Witkin, 1983). In 1984, Koenderink showed the mathematical equivalence between the Gaussian-kernel filtering and the linear diffusion equation, or the partial differential equation of the parabolic type (Koenderink, 1984). The gradient structure of an image in the Gaussian scale space represented by separatrices has been investigated by Griffin and Colchester (Griffin and Colchester, 1995; Griffin, Colchester, and Robinson, 1992). Earlier works on the gradient structure of an image in the Gaussian scale space can be found in (Zhao and Iijima, 1985b), in which the gradient field and curves are called the figure field and the figure-flow curves, respectively.

Scale space simultaneously treats the image at all scales. According to the assumption of socalled scale-space causality (Koenderink, 1984; Lindeberg, 1994), the local structures of an image are supposed to explain every global structure of its coarser, blurred image. The bifurcational properties of image features in the scale space indicate that the structure across the scale, or so-called deep structure (Witkin, 1983; Koenderink, 1984; Kuijper, Florack, and Viergever, 2003; Kuijper, 2002), is hierarchical. The deep structure has been analysed using the trajectories of stationary points in scale space, which are called stationary curves (Zhao and Iijima, 1985a,b) or critical curves including extremum paths (Lifshitz and Pizer, 1990; Lindeberg, 1994; Johansen, Skelboe, Grue, and Andersen, 1986; Lindeberg, 1998; Johansen, 1994; Kuijper, Florack, and Viergever, 2003; Kuijper, 2002). The stationary curves start at the stationary points of the original image, and end at coarser scales where the stationary points are annihilated by blurring. These endpoints are singular points called the top-points (Johansen, Skelboe, Grue, and Andersen, 1986; Johansen, 1994). The adjacencies between the stationary points are also described by the stationary curves since the annihilation events occur at the points where the stationary points meet. However, the hierarchical structure has not been extracted only by the stationary curves. The top-points do not have any connections by the stationary curves to higher scales. To extract the hierarchy, additional connections from the top-points are required; Lifshitz (Lifshitz and Pizer, 1990) and Simmons (Simmons, Arridge, Tofts, and Barker, 1998), for example, have used the connections by isointensity paths, which are the curves in scale space of the same image intensity with the top-points.

To clarify the relationship between local and global structures represented by the stationary points, we observe the behavior not only of the stationary points but also of the gradient structure in the Gaussian scale space. The hierarchical structure in scale space can be investigated in terms of the flow of image intensity, since the diffusion equation can be derived from physical principles of 
flow (Weickert, Ishikawa, and Imiya, 1997; Iijima, 1971; Weickert, Ishikawa, and Imiya, 1999). The spatial gradients can be regarded as the flow with respect to the scale under the diffusion equation. We can trace the flow curve from each top-point and identify the source or drain from which the top-point originates. For topological consistency of the gradient structure, such flow-curve linkage entails the compactification of the space by adding a point at infinity as a representative of the background of the image.

In this study, we firstly redefine the scale space in the compactified domain, and overview the theory on the figure field. We observe the gradient structure over various ranges of scales. The gradient structure expresses the deep structure in the Gaussian scale space by its evolution process with respect to the scale. The process of a two-dimensional image is described as a sequence of pseudo-graphs. At each scale of annihilation, a specific flux curve of the figure field, which we call the antidirectional figure-flow curve, connects the top-point and another local extremum including the point at infinity. Secondly, we analyse the hierarchical structure in the Gaussian scale space by the figure field. The singular point configuration in the Gaussian scale space is called the deep structure of scale space (the DSSS in abbreviation). The DSSS describes the hidden topological nature of the original functions dealing with gray values of an $n$-variable function in the scale space as $(n+1)$-dimensional topographical maps (Zhao and Iijima, 1985a,b; Pelillo, Siddiqi, and Zucker, 1999; Yuille and Poggio, 1986; Kuijper, Florack, and Viergever, 2003; Kuijper and Florack, 2003). The DSSS allows to extract dominant parts of an image and their topological relation. As the DSSS, we present that the hierarchical structure can be derived from the trajectory of singular points across the scales and the antidirectional figure-flow curves at the scales of annihilation. Using the stationary curve, which is the trajectory of singular points across the scales, we propose a method of extracting the hierarchical structure to obtain tree representation.

Discovering objects and detecting their motion from moving images are vital tasks in computer vision. If we restrict the possible target objects and scene depicted by the images, we can accomplish the tasks by modeling the objects and motion within a prescribed tolerance. On the other hand, if we do not have sufficient prior knowledge about the objects and scene, clarifying the unknown objects and scene is a crucial subject in the early stages of vision systems. The apparent structures of the objects in the images are clarified without prior knowledge since we can observe how the image structure is integrated from coarse to fine. We present a scale-space analysis of objects in motion. A sequence of scale-space trees is derived from a temporal sequence of images. Topological transition of the image structure caused by critical changes in the shapes of objects in the image sequence can be detected by the topological difference between the scale-space trees. Consequently, the image sequence is segmented into subsequences without prior knowledge about the objects and their motion in the moving image. We show some experimental results on image sequences to demonstrate advantages of our motion analysis.

Segmentation is a pattern recognition technique for detecting objects in an image. The segmentation of an image with high resolution inherently suffers from the oversegmentation problem, in which invalid segments are misinterpreted as pattern features of the image. In most cases, oversegmentation due to randomness in the image, such as textures and noise, is suppressible by low-pass filtering or smoothing of the image. Therefore, for the segmentation of an image, presmoothing procedure to the image is usually operated. However, smoothing operation reduces the image features as well. The segmentation of the smoothed image fails to extract valid segments related to the target objects in the image, that is, the undersegmentation problem. Therefore, selection of the image resolution is crucial to avoid under- and oversegmentation.

A typical presmoothing is the convolution of an image with a Gaussian kernel with an appropriate variance. Then, a class of differential operations are operated to the presmoothed image for the detection of steepest points as candidates of segment edges. In this process, the variance of the Gaussian kernel, which defines the bandwidth in the Fourier domain, is heuristically selected. The randomness in the image is averaged out as the scale increases. The smoothing process of the image implies that the differential geometric features of the image has hierarchical relationships among them. Therefore, it is expected that the hierarchical relationships among the image segments are deterministic above a certain critical scale even though the image contains random structures.

We introduce a mathematical strategy for the selection of the variance of the Gaussian kernel using the DSSS. Since the stationary points on the stationary curves (Zhao and Iijima, 1985a,b) define dominant parts and their topological relation, we use the topological properties of stationary curves for the selection of the variance of the presmoothing Gaussian kernel. This selection strategy derives the hierarchical structure of the segments in the Gaussian scale space. By reviewing 
differential geometric approaches to edge detection of the image segments from the viewpoint of the scale-space theory, we statistically clarify, in the Gaussian scale space, the smoothing process of the image and evolution of the detected image segments. We show that the critical scale, above which the deterministic image segments are obtained, is estimated from the lifetime of the segments in the Gaussian scale space. We define the hierarchical relationships among the image segments in the Gaussian scale space. According to the hierarchy, every image segment corresponds to one of the deterministic segments in a scale higher than the critical scale. Selecting the scale for segmentation above the critical scale, we can extract the valid segments without a prior knowledge on the number of valid segments and their location.

We also investigate the principal components in the Gaussian scale space of a point cloud ${ }^{2}$. In ref. (Iijima, 1973), Iijima introduced a framework of principal component analysis (PCA) of generalised figures, which are images of an image in the Gaussian scale space. He showed that the Hermite functions are the base function system in the scale space of two-dimensional images. This analytical property of the base functions in the Gaussian scale space is independent of the dimension. In Iijima's book the global properties of the base functions in the scale space treat the modal expression of images in the scale space. In the first one-third of the book, linear scale space theory is dealt with from the view point of observation of two-dimensional images, since "Pattern Recognition" of Iijima was established to introduce a mathematical framework of character recognition ${ }^{3}$.

The Gaussian scale-space theory provides a dimension-independent observation theory for input data. In medical image analysis, three-dimensional extension of the Gaussian scale-space theory is employed to extract the local dimensionality and local directionality of volumetric medical data (Chan, Sato, and Tamura, 2000; Sato, Nakajima, Shiraga, Atsumi, Yoshida, Koller, Gerig, and Kikinis, 1998) by calculating the local mechanical moment of smoothed volumetric data using the spatial Gaussian kernel. This application of the linear scale-space theory in three-dimensional space empirically suggests the possibility of the linear scale-space analysis being a front-end observation tool in higher dimensional data spaces. In recent papers (Chakravarthy and Ghosh, 1996; Roberts, 1997; Nakamura and Kehtarnavaz, 1998; Hinneburg and Keim, 1998; Kothari and Pitts, 1999; Leung, Zhang, and Xu, 2000; Sakai, Imiya, Komazaki, and Hama, 2007), scale-space analysis has been applied to clustering of data in a Euclidean space of arbitrary dimension. Their clustering strategies can be interpreted to be estimations of data distribution and its structure in a scale space of multidimension.

As a sequel to the clustering method using scale-scale analysis, we develop a local principal component analysis in the Gaussian scale space. This analysis evaluates local dimensionalities and directionalities of clusters in a point cloud in a Euclidean space of arbitrary dimension (Imiya, Ootani, and Kawamoto, 2004; Imiya and Kawamoto, 2001). Imiya et al (Imiya, Ootani, and Kawamoto, 2004), extracted the dimensionalities and directionalities of the clusters of a point cloud using the voting-based learning algorithm. In (Chakravarthy and Ghosh, 1996; Roberts, 1997; Nakamura and Kehtarnavaz, 1998; Hinneburg and Keim, 1998; Kothari and Pitts, 1999; Leung, Zhang, and Xu, 2000; Sakai, Imiya, Komazaki, and Hama, 2007), they showed that the Gaussian scale-space theory clarify hierarchy among the clusters and constructed the scale-space-based algorithms for the determination of the number of clusters in a point cloud. In this study, we develop a framework to extract the clusters in a point cloud and to evaluate their statistical significance or cluster validity. This treatment clarifies the dimensionalities, principal directions, and hierarchical relations of valid clusters in a point cloud under the uncertainty of the spatial resolution of observation. According to the scale-space theory, such uncertainty is axiomatically approximated by the Gaussian kernel.

\section{Basics of Scale Space}

\subsection{Gaussian Scale Space}

We define the image as a non-negative scalar function of one or more variables defined in the extended real space such that $\overline{\mathbb{R}}^{N}=\mathbb{R}^{N} \cup\{\infty\}$ for the $N$-dimensional Euclidean space $\overline{\mathbb{R}}^{N}$ and a point at infinity $\{\infty\}$ in $\overline{\mathbb{R}}^{N}$, where $N \geq 1$.

\footnotetext{
${ }^{2}$ In this study, we call a set of points distributed in a space of arbitrary dimension a point cloud. A point cloud is a set of points in the three-dimensional Euclidean space for shape modeling, and is a set of feature values in a feature space of arbitrary dimension for manifold learning.

${ }^{3}$ IIjima called his mathematical theory for analysis and recognition of patterns and images using Gaussian filtering and the linear diffusion equation the general theory of pattern recognition based on the theory of pattern.
} 
Definition 1 An image is defined as a non-negative scalar function $f(\boldsymbol{x}), \boldsymbol{x} \in \overline{\mathbb{R}}^{N}$.

Although the domain of a grey-scale image is practically bounded within a limited area or volume, we embed such an image in the extended real space to apply our theory. Scale space is known to be a set of blurred images obtained by the conversion of a scale or an inverse resolution of the original image $f(\boldsymbol{x})$. A one-parameter family of positive function $f(\boldsymbol{x}, \tau)$ is derived from the blurring filter with a single control parameter $\tau$. The $N+1$ dimensional space $(\boldsymbol{x}, \tau)$ is called the scale space. It was Iijima (Iijima, 1962, 1963, 1971, 1973, 1999) who first presented an axiomatic derivation of the Gaussian scale space. For a blurring operation $\Phi$, The scale space axioms are as follows -

Non-negative intensity of images. A blurred positive image is positive:

$$
\Phi \geq 0
$$

Linearity of image intensity. Blurring an illuminated image is the same operation as illuminating the blurred image:

$$
\Phi[A f, \boldsymbol{x}, \tau]=A \Phi[f, \boldsymbol{x}, \tau] .
$$

Closedness under affine transformations. Blurring an affine-transformed image is the same operation as affine transformation of the blurred image:

$$
\Phi[f(\boldsymbol{A} \boldsymbol{x}+\boldsymbol{b}), \boldsymbol{x}, \tau]=\Phi\left[f(\boldsymbol{x}), \boldsymbol{A} \boldsymbol{x}+\boldsymbol{b}, \tau^{\prime}\right],
$$

where $\tau^{\prime}$ is a value depending on $\tau$ and $\boldsymbol{A}$. The affine transformation can be expansion, contraction, rotation, or shift in position.

Associative (or semigroup) property. There exists a single blurring with a scale $\tau$ equivalent to successive blurring with $\tau_{1}$ and $\tau_{2}$ :

$$
\Phi\left[\Phi\left[f, \boldsymbol{x}, \tau_{1}\right], \boldsymbol{x}, \tau_{2}\right]=\Phi[f, \boldsymbol{x}, \tau],
$$

where $\tau$ is the scale depending on $\tau_{1}$ and $\tau_{2}$.

These axioms lead to the convolution

$$
\Phi[f, \boldsymbol{x}, \tau]=G * f
$$

with an isotropic Gaussian function

$$
G(\boldsymbol{x}, \tau)=\frac{1}{\sqrt{4 \pi \tau}^{N}} \exp \left(-\frac{|\boldsymbol{x}|^{2}}{4 \tau}\right) .
$$

The scale $\tau$ is related to the width of the Gaussian function. Later, the derivation of the Gaussian kernel for scale-space filtering was independently proposed by Witkin (Witkin, 1983; Badaud, Witkin, Baudin, and Duda, 1986) and Koenderink (Koenderink, 1984).

For motion analysis, the recognition of target objects in the image and their motion should be independent of motion of the observer. The translation and rotation invariance in the axioms are suitable for the detection of structural changes of the objects.

In this study, we deal with the Gaussian scale space in the extended real scale and space. The point at infinity and the infinite scale play essential roles in the structural analysis of the image in scale space.

Definition 2 The Gaussian scale-space image $f(\boldsymbol{x}, \tau),(\boldsymbol{x}, \tau) \in\left(\overline{\mathbb{R}}^{N}, \overline{\mathbb{R}}^{+}\right)$is the convolution of the $N$-dimensional original image $f(\boldsymbol{x}) \geq 0$ with the isotropic Gaussian kernel $G(\boldsymbol{x}, \tau)$.

The Gaussian kernel in Eq. (6) is normalised so that the scale-space image $f(\boldsymbol{x}, \tau)$ satisfies the linear diffusion equation

$$
\frac{\partial}{\partial \tau} f(\boldsymbol{x}, \tau)=\Delta f(\boldsymbol{x}, \tau), \quad f(\boldsymbol{x}, 0)=f(\boldsymbol{x}) .
$$

That is, the Gaussian scale-space image $f(\boldsymbol{x}, \tau)$ is a diffused version of the original image $f(\boldsymbol{x})$ in the infinite domain. As the features of the original image are reduced with increasing scale, the structure of the image is simplified. This process is illustrated in Fig. 1. In later sections, we show that the structural simplification of the image can be wholly provided by the spatial gradient of $f(\boldsymbol{x}, \tau)$. 


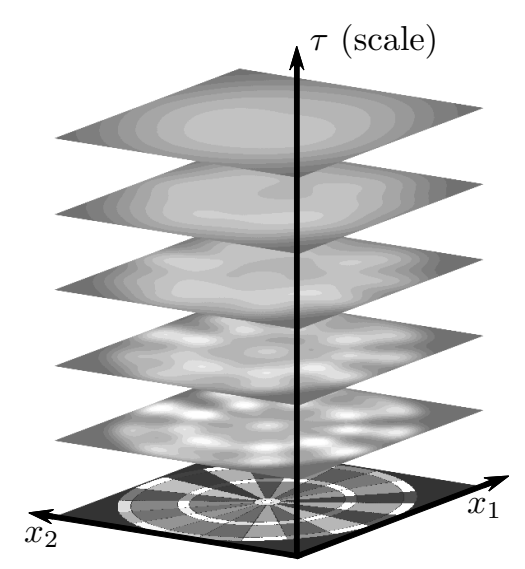

Figure 1: Image in the Gaussian scale space. As the scale increases, the original image at the bottom is blurred and the structure is simplified.

\subsection{Stationary Points}

We focus on stationary points, or so-called critical points, of the blurred image, since the stationary points are well-defined feature points of the scalar function $f(\boldsymbol{x}, \tau)$ in the sense of the differential geometry.

Definition 3 Stationary points are defined as points where the spatial gradient vanishes.

$$
\{\boldsymbol{x} \mid \nabla f(\boldsymbol{x}, \tau)=\mathbf{0}\}
$$

The stationary points can be considered as geometric cues. Figure 2(a) shows an example of the stationary points of a two-dimensional image at a fixed scale. The local maxima are representative of bright image components. The local minima correspond to dark cavities in the image. The saddle points appear between the local extrema and imply the presence of connections between them. Topographically, the saddle points of the two-dimensional image are feature points on ridges and troughs, which connect the local maxima and local minima, respectively. Note that the point at infinity is a hidden local minimum for a positive image $f(\boldsymbol{x})$, since any small displacement from the point at infinity increases the intensity of the diffused image $f(\boldsymbol{x}, \tau)>0$ at the point $\boldsymbol{x} \in \mathbb{R}^{N}$. The local minimum at infinity represents the dark background of the positive image.

We have regular and singular stationary points. The regular stationary points of the $N$-dimensional $(N>1)$ image are classified into three types: a local maximum, a local minimum, and a saddle point. They are discriminated by the second derivative test. Since the directional derivative of $f(\boldsymbol{x}, \tau)$ in the direction of a spatial unit vector $\boldsymbol{n}$ is calculated as

$$
\frac{d f}{d n}=\boldsymbol{n}^{\top} \nabla f,
$$

the second directional derivative of $f(\boldsymbol{x}, \tau)$ can be written in the quadratic form

$$
D^{2}(\boldsymbol{n})=\frac{d^{2} f}{d n^{2}}=\boldsymbol{n}^{\top} \boldsymbol{H} \boldsymbol{n} .
$$

The function $f(\boldsymbol{x}, \tau)$ is said to be convex if the second directional derivative $D^{2}(\boldsymbol{n})$ is positive for any direction of $\boldsymbol{n}$. Analogously, $f(\boldsymbol{x}, \tau)$ is concave for negative $D^{2}(\boldsymbol{n})$. The local maximum and local minimum are the stationary points at the concave and convex parts of the function $f(\boldsymbol{x}, \tau)$, respectively.

The eigenvalues of $\boldsymbol{H}$ and the corresponding eigenvectors are called the principal curvatures and the principal directions, respectively. Setting $\lambda_{i}(i \leq N, i \in \mathbb{N})$ be the eigenvalues of $\boldsymbol{H}$, we set $\lambda_{\max }$ and $\lambda_{\min }$ the maximum and minimum eigenvalues, respectively, among them. Then, Eq. (10) suggests

$$
\lambda_{\min } \leq \frac{d^{2} f}{d n^{2}} \leq \lambda_{\max } .
$$




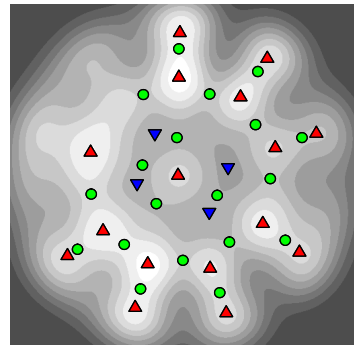

(a)

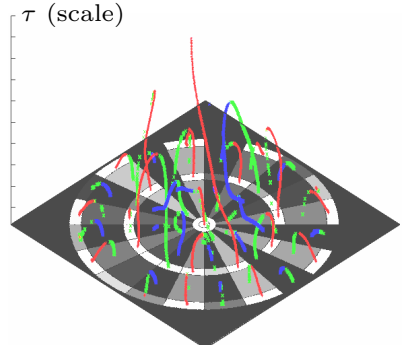

(b)

Figure 2: Stationary points and curves $(N=2)$. (a) Stationary points of an image. Local maxima, local minima, and saddle points are indicated by the upward triangles, downward triangles, and circles, respectively. (b) Trajectories of the stationary points in scale space.

Therefore, if $\boldsymbol{H}$ is negative definite at a stationary point $\mathrm{P}$, then $\mathrm{P}$ is a local maximum, and all the eigenvalues are negative $\left(\lambda_{\max }<0\right)$. Conversely, if $\boldsymbol{H}$ is positive definite at a stationary point $\mathrm{P}$, then $\mathrm{P}$ is a local minimum, and all the eigenvalues are positive $\left(\lambda_{\min }>0\right)$. Otherwise, the stationary point is a saddle point. We denote the signs of the eigenvalues of $\boldsymbol{H}$ as $( \pm, \pm, \ldots, \pm)$. In 3D images, for example, there exist two types of saddle point: an attracting saddle and a repelling saddle (Theisel, Weinkauf, Hege, and Seidel, 1986). They are schematically illustrated in Fig. 3. The attracting saddle has two negative eigenvalues of $\boldsymbol{H}=\nabla \nabla^{\top} f$, and the repelling saddle has two positive eigenvalues.

The saddle point is further classified into three types on the basis of the sign of

$$
\Delta f=\operatorname{tr} \boldsymbol{H}=\operatorname{tr}\left(\boldsymbol{V} \boldsymbol{\Lambda} \boldsymbol{V}^{\top}\right)=\sum \lambda_{i},
$$

where $\boldsymbol{V}$ is the square matrix whose column vectors are eigenvectors of $\boldsymbol{H}$, and $\Lambda$ is the diagonal matrix of the eigenvalues $\lambda_{i}$. For two-dimensional images, these types are called the ridge-like $(\Delta f<0)$, trough-like $(\Delta f>0)$, and balanced $(\Delta f=0)$ saddles (Griffin and Colchester, 1995). The balanced saddle is also known as the scale-space stationary point since the spatial gradient and scale derivative $\partial f / \partial \tau$ simultaneously vanish (Kuijper, Florack, and Viergever, 2003). Equations (7) and (12) indicate that the scale derivative can be zero only at stationary points with positive and negative eigenvalues. Therefore, the scale-space stationary point is always a spatial saddle point.

The singular stationary points are also called the catastrophe points (Kuijper, Florack, and Viergever, 2003; Kuijper and Florack, 2005) in the scale-space theory.

Definition 4 Catastrophe points are the points where both the spatial gradient and the determinant of the Hessian matrix vanish.

$$
\{(\boldsymbol{x}, \tau) \mid \nabla f(\boldsymbol{x}, \tau)=\mathbf{0} \text { and } \operatorname{det} \boldsymbol{H}=0\}
$$

Every singular point generically has a zero eigenvalue, or a zero principal curvature since

$$
\operatorname{det} \boldsymbol{H}=\prod \lambda_{i}=0 .
$$

The singular points are the points at which the stationary points meet in the scale space (Florack and Kuijper, 2000). This property is discussed in the next section.

\subsection{Stationary Curves}

As the scale changes, the stationary points of the diffused image move in the space. Observing these stationary points in the scale space, we see their trajectories across scales. We call them the stationary curves, also known as the critical curves.

Definition 5 Stationary curves are the trajectories of stationary points in scale space.

The stationary curves are also classified as local maximum curves, local minimum curves, and saddle curves. 


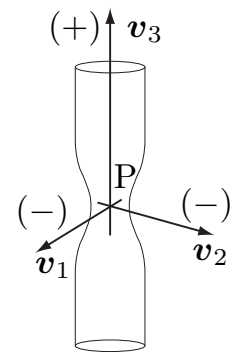

(a)

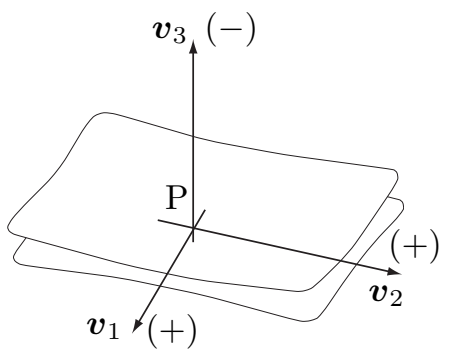

(b)

Figure 3: Two types of saddle point in 3D images. (a) Attracting saddle $(-,-,+)$. The saddle point $\mathrm{P}$ with one positive and two negative eigenvalues of $\boldsymbol{H}$ lies in a narrow part of the image resembling a line. (b) Repelling saddle $(+,+,-)$. The saddle point $\mathrm{P}$ with two positive and one negative eigenvalues resides in a sheetlike image.

The motion of the stationary points with respect to the scale is described by the stationary curves. Since the saddle point exists between the local extrema, the disappearance of a local extremum of the diffused image is always accompanied by that of a saddle point. As shown in Fig. 2(b), local extrema are annihilated when they meet saddle points with increasing scale. It is noteworthy that a local extremum curve and a saddle curve share the top endpoint. Therefore, the connections of the stationary curves indicate topological relationships among the local extrema and saddle points.

The stationary curves are denoted by one-dimensional manifolds $\boldsymbol{x}(\tau)$ in the scale space. A total differential equation of $\nabla f(\boldsymbol{x}, \tau)$ is written as

$$
\frac{d}{d \tau} \nabla f=\boldsymbol{H} \frac{d \boldsymbol{x}}{d \tau}+\frac{\partial}{\partial \tau} \nabla f
$$

Since $\nabla$ and $\partial / \partial \tau$ are commutable, and $\nabla f=\mathbf{0}$ at the critical point $\boldsymbol{x}(\tau)$, we have the equation

$$
\boldsymbol{H} \frac{d \boldsymbol{x}(\tau)}{d \tau}=-\nabla \frac{\partial}{\partial \tau} f(\boldsymbol{x}(\tau), \tau)
$$

Considering the diffusion equation of Eq. (7) in the Gaussian scale space, Zhao and Iijima (Zhao and Iijima, 1985a,b) showed that the stationary curves are solutions to the system of differential equations

$$
\boldsymbol{H} \frac{d \boldsymbol{x}(\tau)}{d \tau}=-\nabla \Delta f(\boldsymbol{x}(\tau), \tau)
$$

The ordinary derivative of $\boldsymbol{x}(\tau)$ on the left-hand side of Eq. (17) is the spatial velocity of a stationary point with respect to the scale. Therefore, Eq. (17) indicates that the velocity of the stationary point is determined by the spatial derivatives of second order $\boldsymbol{H}$ and third order $\nabla \Delta f$ of the scale-space image.

Florack and Kuijper (Florack and Kuijper, 2000) discussed the velocity of the stationary point and the local structure in detail. Equation (17) is solvable if $\boldsymbol{H}$ is invertible. At singular points, the velocity is infinite in the direction of the zero principal curvature. For these reasons, the top endpoints of the stationary curves are the singular points, and the stationary curves are smoothly connected at the singular points.

Transforming the coordinates into the principal axis coordinates of $\boldsymbol{H}$, we can rewrite Eq. (17) as

$$
\frac{d \boldsymbol{p}}{d \tau}=-\boldsymbol{\Lambda}^{-1} \nabla_{p} \Delta f
$$

for $\boldsymbol{p}(\tau)=\boldsymbol{V}^{\top} \boldsymbol{x}(\tau)$, where $\nabla_{p}=\boldsymbol{V}^{\top} \nabla$ is the gradient operator in the principal axis coordinates.

According to the catastrophe theory in the Gaussian scale space (Kuijper, 2002; Damon, 1995, 1997), fold catastrophes describe generic annihilation events. In the principal axis coordinates, the annihilation event is modelled as

$$
f(\boldsymbol{p}, s)=p_{1}^{3}+6 p_{1} s+\sum_{i=2}^{N} \gamma_{i}\left(p_{i}+2 s\right)
$$


where $\sum_{i=2}^{N} \gamma_{i} \neq 0$ and $\forall \gamma_{i} \neq 0$. Since $s$ is the scale parameter, the annihilation event occurs at $s=0$. For $N$-dimensional $(N>1)$ images, it is sufficient to consider the catastrophes in a two-dimensional case described as

$$
f\left(p_{1}, p_{2}, s\right)=p_{1}^{3}+6 p_{1} s+\gamma\left(p_{2}^{2}+2 s\right)
$$

This model of the scale-space image $f\left(p_{1}, p_{2}, s\right)$ has a local maximum and a saddle point if $s<0$ and $\gamma<0$. These two stationary points meet at the origin at $s=0$. The parameterised stationary curves are obtained from Eqs. (18) and (20) as

$$
\boldsymbol{p}(s)=( \pm \sqrt{-2 s}, 0)^{\top}
$$

where the signs + and - correspond to the saddle curve and local maximum curve, respectively. The principal curvatures $\left(\lambda_{1}, \lambda_{2}\right)$ are $(\sqrt{-2 s}, 2 \gamma)$ on the saddle curve and $(-\sqrt{-2 s}, 2 \gamma)$ on the local maximum curve. Therefore, the zero principal curvature direction at the annihilation scale $s=0$ is in the $p_{1}$-axis.

The scale-space image has at least one local maximum at any scale. There exists one remaining local maximum at the coarsest scale.

Definition 6 A trunk curve is a local maximum curve connected to the one remaining local maximum at the coarsest scale.

The trunk curve starts at one of the local maxima at the finest scale, and ends at the one remaining local maximum at the coarsest scale.

Proposition 1 The one remaining local maximum converges to the centroid of the original image as the scale increases (Zhao and Iijima, 1985b).

\section{Proof 1}

$$
\begin{aligned}
\nabla f(\boldsymbol{x}, \tau) & =\nabla(G(\boldsymbol{x}, \tau) * f(\boldsymbol{x}))=(\nabla G(\boldsymbol{x}, \tau)) * f(\boldsymbol{x}) \\
& =(\boldsymbol{x} G) * f(\boldsymbol{x})-\boldsymbol{x}(G * f(\boldsymbol{x}))=0 .
\end{aligned}
$$

Therefore,

$$
\boldsymbol{x}=\frac{(\boldsymbol{x} G) * f(\boldsymbol{x})}{(G * f(\boldsymbol{x}))} \rightarrow \frac{\boldsymbol{x} * f(\boldsymbol{x})}{1 * f(\boldsymbol{x})} \quad(\tau \rightarrow \infty) .
$$

Note that the local minimum at infinity also resides at any scale. The collection of local minima at infinity in the scale space can be regarded as a local minimum curve.

Definition 7 A local minimum curve at infinity is a collection of local minima at infinity in the scale space.

The trunk curve and the local minimum curve at infinity are the two capital stationary curves in scale space spanning from the finest scale to the coarsest scale. We presume that the trunk curve and the local minimum curve at infinity are virtually connected at an infinite scale, since the scale-space image is completely flat and no stationary point is found at the infinite scale.

\section{$2.4 \quad$ Figure Field}

The stationary points of images are detected as zeros of the spatial gradient. While the stationary curves indicate the topological relationships among the stationary points, the pattern of the spatial gradient field clarifies the topological structure explicitly. The spatial gradient field of the scalespace image is known as the figure field, and its field curves are called the figure-flow curves (Zhao and Iijima, 1985b).

Definition 8 The figure field $\boldsymbol{F}$ is defined as the negative of the vector field of the scale-space image.

$$
\boldsymbol{F}=-\nabla f(\boldsymbol{x}, \tau)
$$

Definition 9 The figure-flow curves are the directional flux curves of the figure field. 


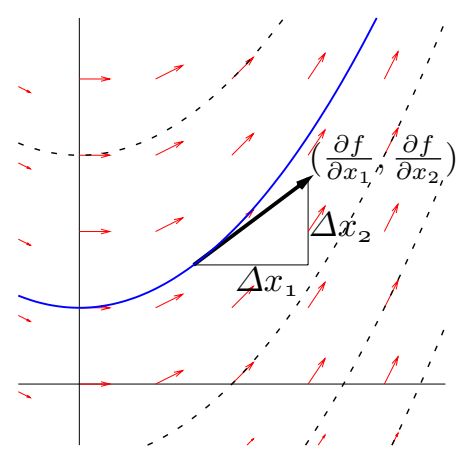

Figure 4: Schematic illustration of eq. (24).

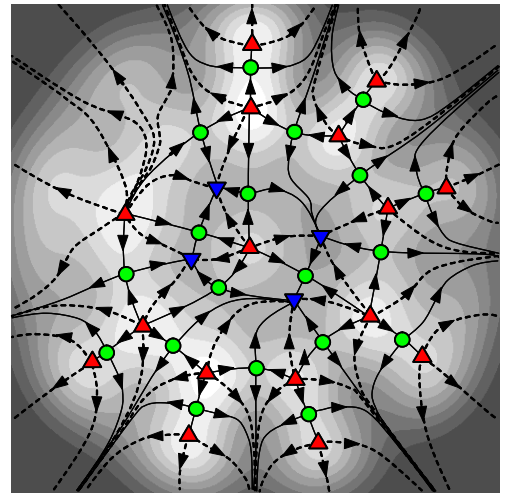

Figure 5: Figure field of the image in Fig. 2(a).

For two-dimensional images, the figure-flow curves are the set of solutions to the differential equation

$$
\frac{d x_{2}}{d x_{1}}=\frac{\frac{\partial f}{\partial x_{2}}}{\frac{\partial f}{\partial x_{1}}}
$$

Figure 4 shows the geometrical interpretation of Eq. (24). The figure field can be considered current density flow of the image intensity with respect to scale, since the figure field satisfies the continuity equation.

Proposition 2 The figure field $\boldsymbol{F}$ satisfies the equation of continuity

$$
\frac{\partial f}{\partial \tau}+\nabla^{\top} \boldsymbol{F}=0
$$

Equation (25) is directly obtained from Eqs. (7) and (23).

Figure 5 shows an example of the figure-flow curves of the image in Fig. 2(a) at a fixed scale. The local maxima and local minima are sources and drains of the flow of image intensity, respectively. We can draw as many figure-flow curves as possible from sources to drains. In Fig. 5 figure-flow curves are indicated by the dashed curves. The saddle points are confluent points of two inward and two outward figure-flow curves. The figure-flow curves through the saddle points are called separatrices. Since the divergence of the figure field is $\nabla^{\top} \boldsymbol{F}=-\nabla^{\top} \nabla f=-\Delta f$, the net incoming image intensity at ridge-like saddles is negative, and that at trough-like saddles is positive. Recall that we defined the image in the extended real space. The outward figure-flow curves which meet the boundary of the image displayed in Fig. 5 are extended beyond the boundary and are considered to end at the point at infinity. This implies that the local minimum at infinity is a drain of the flow of image intensity from the whole domain of the positive image. 

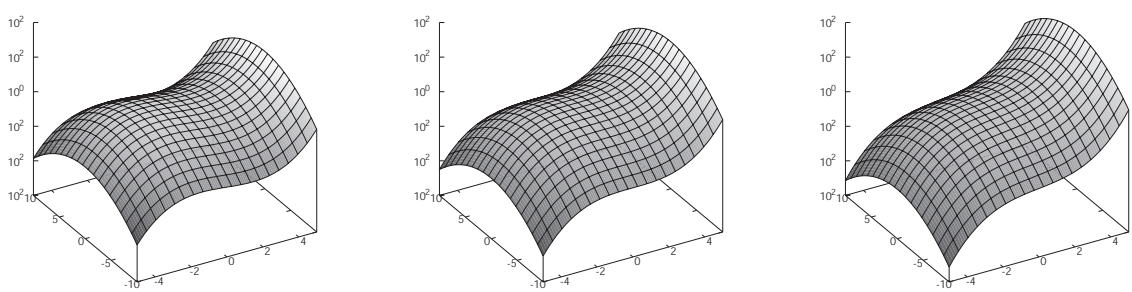

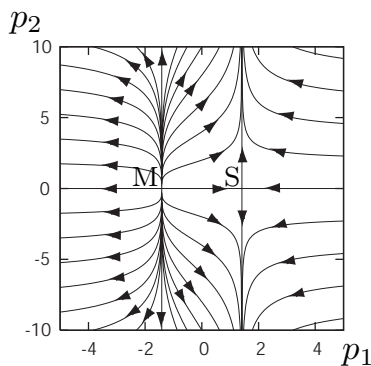

(a)

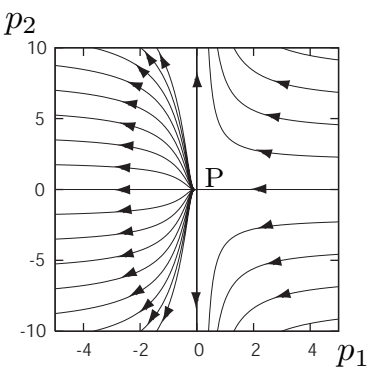

(b)

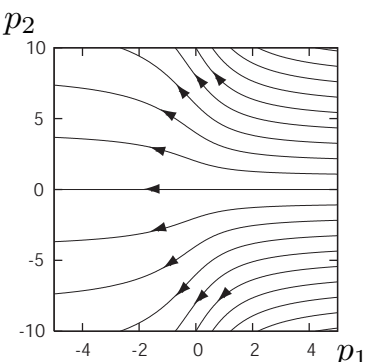

(c)

Figure 6: Surface plots of $f\left(p_{1}, p_{2}\right)$ and corresponding figure flow curves (a) before, (b) at, and (c) after the fold catastrophe event.

\section{Scale-Space Hierarchy}

In the previous section, we argued that the connections of the stationary curves indicate topological relationships among the stationary points or the feature points of an image. If the relationships among the stationary points are hierarchical, we can determine the hierarchical structure of the image. The hierarchical structure, however, is still unclear because the top endpoints, which are the annihilation points, do not have connections by the stationary curves, to any points in higher scales. The points to which the annihilation points are connected cannot be identified only by the stationary curves. We require connections of the annihilation points in addition to the stationary curves to determine the hierarchy.

One possibility for the additional connections is isointensity paths proposed in ref. (Lifshitz and Pizer, 1990). The isointensity paths are the curves in scale space with the same image intensity of the top endpoints, and connect the top endpoints to other local extrema. However, we require both the spatial gradient and the image intensity to explain the hierarchical structure.

In this section, we show a nonheuristic and nonempirical approach to resolving the hierarchical structure of an image in scale space only using the spatial gradient. We exhibit evolution of the figure-flow curves in the vicinity of an annihilation point. We find that a specific figure-flow curve is related to the annihilation point of a local extremum and a saddle point. This figure-flow curve identifies the point to which the annihilation point is connected in the hierarchy. The local minimum at infinity is also involved in the hierarchical connection.

\subsection{Figure Field in Annihilation Event}

A significant aspect of the annihilation event is the evolution of the figure field. Noting that $\nabla_{p}=$ $\boldsymbol{V}^{\top} \nabla$ and replacing $\tau$ with $s$, it follows, from Eqs. (20) and (23), that

$$
\boldsymbol{F}=-\left(3 p_{1}^{2}+6 s, 2 \gamma p_{2}\right)^{\top} .
$$

A family of figure-flow curves $p_{2}=C\left(p_{1}\right)$ is derived from Eq. (24). The solution for $f\left(p_{1}, p_{2}, s\right)$ is

$$
p_{2}=\left\{\begin{array}{cc}
A\left|\frac{p_{1}-\sqrt{-2 s}}{p_{1}+\sqrt{-2 s}}\right|^{\frac{\gamma}{3 \sqrt{-2 s}}} & (s<0) \\
A \exp \left(-\frac{2 \gamma}{3 p_{1}}\right) & (s=0) \\
A \exp \left(\frac{2 \gamma}{3 \sqrt{2 s}} \tan ^{-1} \frac{p_{1}}{\sqrt{2 s}}\right) & (s>0) .
\end{array}\right.
$$




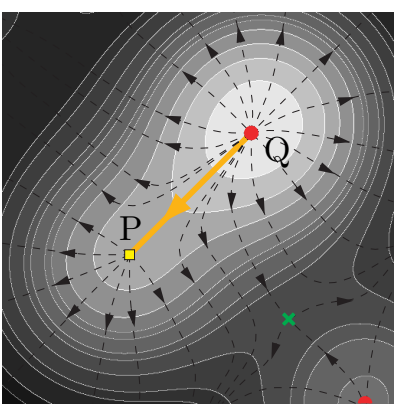

(a)

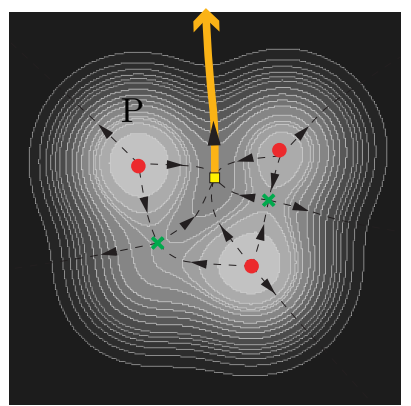

(b)

Figure 7: Contour maps of scale-space image and figure-flow curves at annihilation scale. (a) Annihilation of local maximum. The antidirectional figure-flow curve (solid line) links the annihilation point $\mathrm{P}$ and the local maximum Q. (b) Annihilation of local minimum. The antidirectional figureflow curve to the boundary of the image indicates the connection to the point at infinity.

Figure 6 shows plots of the annihilation of a local maximum $\mathrm{M}$ and a saddle $\mathrm{S}$. In the upper panels are the surface plots of the image intensity, before, at, and after the annihilation event. In the lower panels show the corresponding figure-flow curves calculated as Eq. (27). The stationary points $\mathrm{M}$ and $\mathrm{S}$ in Fig. 6(a) meet at the annihilation point $\mathrm{P}$ in Fig. 6(b) with increasing scale. Outward figure-flow curves start at the annihilation point $\mathrm{P}$, and, only one inward figure-flow curve ends at $\mathrm{P}$. Here, we call it the antidirectional figure-flow curve.

Definition 10 A nongeneric figure-flow curve starts or ends at the singular point. An antidirectional figure-flow curve is defined as the figure-flow curve which ends at the annihilation point of the local maximum and saddle, or starts at the annihilation point of the local minimum and saddle.

We clearly see that the antidirectional figure-flow curve coincides with the zero principal curvature direction, the $p_{1}$-axis.

\subsection{Connection by Antidirectional Figure-Flow Curve}

The motion of the stationary points described by Eq. (17) suggests the following geometrical properties of the annihilation point.

- The annihilation point is singular.

- The velocity of a stationary point is infinite at the annihilation point.

- The direction of the velocity of a stationary point is in that of the zero principal curvature at the annihilation point.

Considering the infinite velocity at the annihilation point, the antidirectional figure-flow curve can be regarded as a continuation of the stationary curve at the annihilation scale.

The antidirectional figure-flow curve connects the annihilation point and another local extremum. The connection of the annihilation point and a local maximum is illustrated in Fig. 7(a). Since we can regard the figure field as the density flow of the image intensity with respect to the scale, the image intensity of the annihilation point $\mathrm{P}$ is provided only by the local maximum $\mathrm{Q}$ at the annihilation scale. Figure 7(b) shows an example of the connection to the local minimum at infinity. In the extended real space, the antidirectional figure-flow curve which meets the boundary of the image is ended at the local minimum at infinity. Therefore, the local minimum at infinity is identified as the parent of the annihilation point $\mathrm{P}$ in Fig. 7(b). Figure 8(a) and (b) shows a merging process of blobs. (a)shows the contour map and gradient field curves at the merging scale. The annihilated local maximum $\mathrm{P}$ suddenly falls into a larger blob with the local maximum Q as shown in Fig. 8(b). Then, the antidirectional figure-flow curve (solid line) links $\mathrm{P}$ and Q. Therefore, the local maximum $\mathrm{Q}$ is identified as the parent of the annihilation point $\mathrm{P}$ as shown in Fig. 9. In the same manner as the local maximum, the antidirectional figure-flow curve identifies a local minimum as the parent of the annihilation point of a local minimum and a saddle point. It is notable that the connection of the local minima involves the local minimum at infinity. 


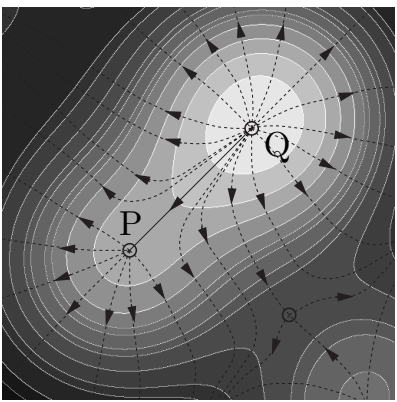

(a)

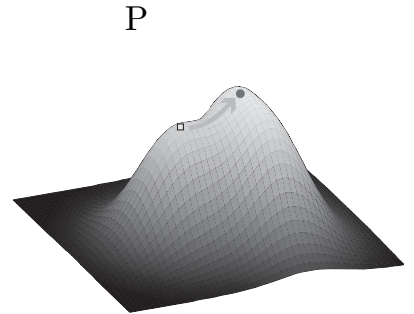

(b)

Figure 8: Merging blobs. (a) The contour map and gradient field curves at the merging scale. (b) The annihilated local maximum $\mathrm{P}$ suddenly falls into a larger blob with the local maximum Q. The antidirectional figure-flow curve (solid line) links P and Q.

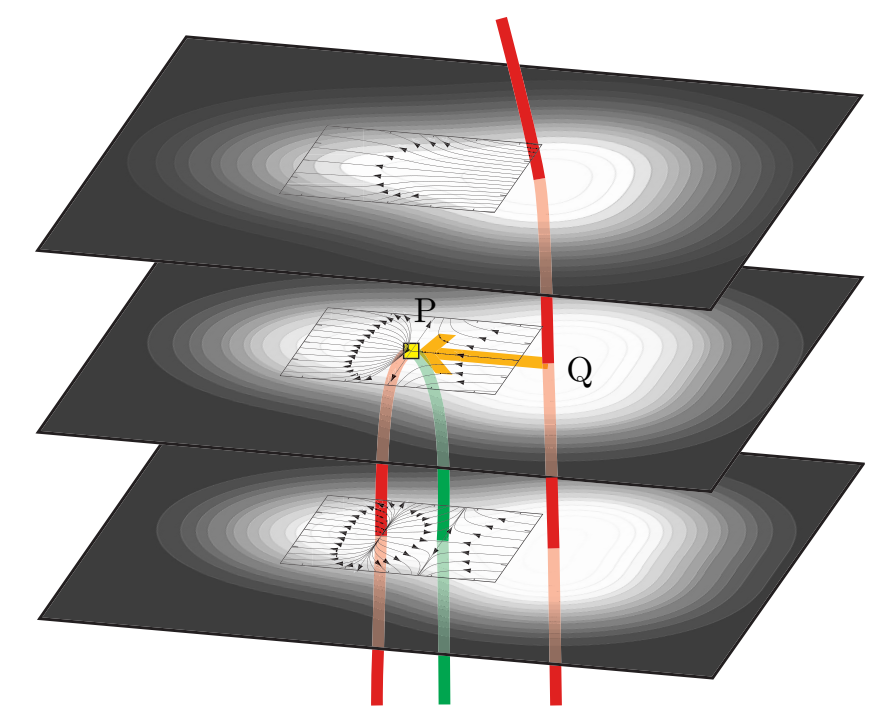

Figure 9: Scale-space hierarchy. The connections by the stationary points and the antidirectional figure-flow curve define the hierarchical structure of the image.

\subsection{Hierarchical Structure of Image}

The hierarchical structure of an image is wholly provided by the spatial gradient. As discussed in the previous sections, the stationary curves in scale space describe the pair annihilation of the local extremum and saddle point, and determine the topology of the stationary points. The antidirectional figure-flow curve at each annihilation scale assigns a parent local extremum to the annihilation point. Thus, the stationary curves and figure-flow curves, which are obtained from the spatial gradient of the image in scale space, define the hierarchical structure.

\section{Scale-Space Tree}

\subsection{Tree Representation}

The scale-space hierarchy derived from the spatial gradient of the image is described as a tree, symbolically. We define the scale-space tree as follows.

Definition 11 The scale-space tree of an image is a tree of which

- the leaves correspond to the stationary points of the image at the finest scale,

- the branches represent the stationary curves in the scale space and the antidirectional figureflow curves at annihilation scales, 
- the nodes represent the annihilation points and the local extrema at which the antidirectional figure-flow curves start or end,

- the root stands for the virtual annihilation point of the local minimum at infinity and a local maximum at the infinite scale.

An example of the top-down process of tree construction is shown in Fig. 10. As the scale decreases, the scale-space image is structured, and a corresponding scale-space tree branches out. The nodes are appended at each annihilation scale.

Generically, the scale-space tree is a binary tree. Since the generic annihilation point of a local extremum is always connected to the same type of local extremum, the resulting tree consists of two subtrees: one with nodes for local maxima and their annihilation points only, and the other with nodes for local minima and their annihilation points only. The subtree for local maxima has branches which compose the trunk curve, and the subtree for local minima contains branches which indicate the local minimum curve at infinity. They are rooted at a node which represents the virtual annihilation at an infinite scale.

\subsection{Tree Construction}

As shown in Fig. 10, the scale-space tree is constructed in a top-down fashion by the coarse to fine observation of an image. In practice, the scale-space image is obtained by diffusion computation in increasing order of the scale. Therefore, an algorithm for bottom-up construction of the tree is desirable to yield the scale-space image and tree simultaneously. We present such an algorithm of constructing a scale-space tree $T$ for a given image $f(\boldsymbol{x})$.

\section{ALGORITHM I - Scale-Space Tree}

1. Set nodes for stationary points at an initial scale $\tau=\tau_{0}$ to be leaves of a tree $T$.

2. Diffuse the scale-space image $f\left(\boldsymbol{x}, \tau_{n-1}\right)$ to yield $f\left(\boldsymbol{x}, \tau_{n}\right)$.

3. Detect annihilation events of local extrema and saddles within $\tau_{n-1} \leq \tau \leq \tau_{n}$, by examining links of the stationary points between the subsequent scales $\tau_{n-1}$ and $\tau_{n}$.

4. For each detected annihilation event

(4-i) Add to $T$ a new node with two branches leading to nodes for the local extremum and saddle involved in annihilation.

(4-ii) For the annihilation event of the local maximum, maximise $f\left(\boldsymbol{x}, \tau_{n}\right)$ with the annihilation point as the initial point.

(4-iii) For the annihilation event of the local minimum, minimise $f\left(\boldsymbol{x}, \tau_{n}\right)$ with the annihilation point as the initial point.

(4-iv) Regard the annihilation point as having a connection to a local minimum at infinity if minimisation results in a point on the boundary of the image.

(4-v) Add to $T$ a new node with two branches. One branch is attached to the node for the annihilation point. The other branch is attached to a node for the local extremum determined by the maximisation or minimisation.

5. Return to step 2 until there remains no saddle point.

6. Add to $T$ a node for the virtual annihilation point of one remaining local maximum and the local minimum at infinity.

According to the definition of the scale-space tree, we need to find parent-child relationships among the singular and regular stationary points. At the scale of annihilation, not the form, but the connection by the antidirectional figure-flow curve is essential in constructing the tree. The parentchild relationships are found in the maximisation and minimisation processes described in (4-ii) and (4-iii). Since the initial direction at the annihilation point to maximise or minimise $f(\boldsymbol{x}, \tau)$ can be found along the antidirectional figure-flow curve, it is apparent that the local search from the annihilation point converges to the local extremum to which the annihilation point is connected.

For the purpose of further image analysis, the nodes of the tree $T$ may preserve geometric information on the scale-space image at the stationary points in scale space, such as positions, scales, intensities, and sets of the principal curvatures. 

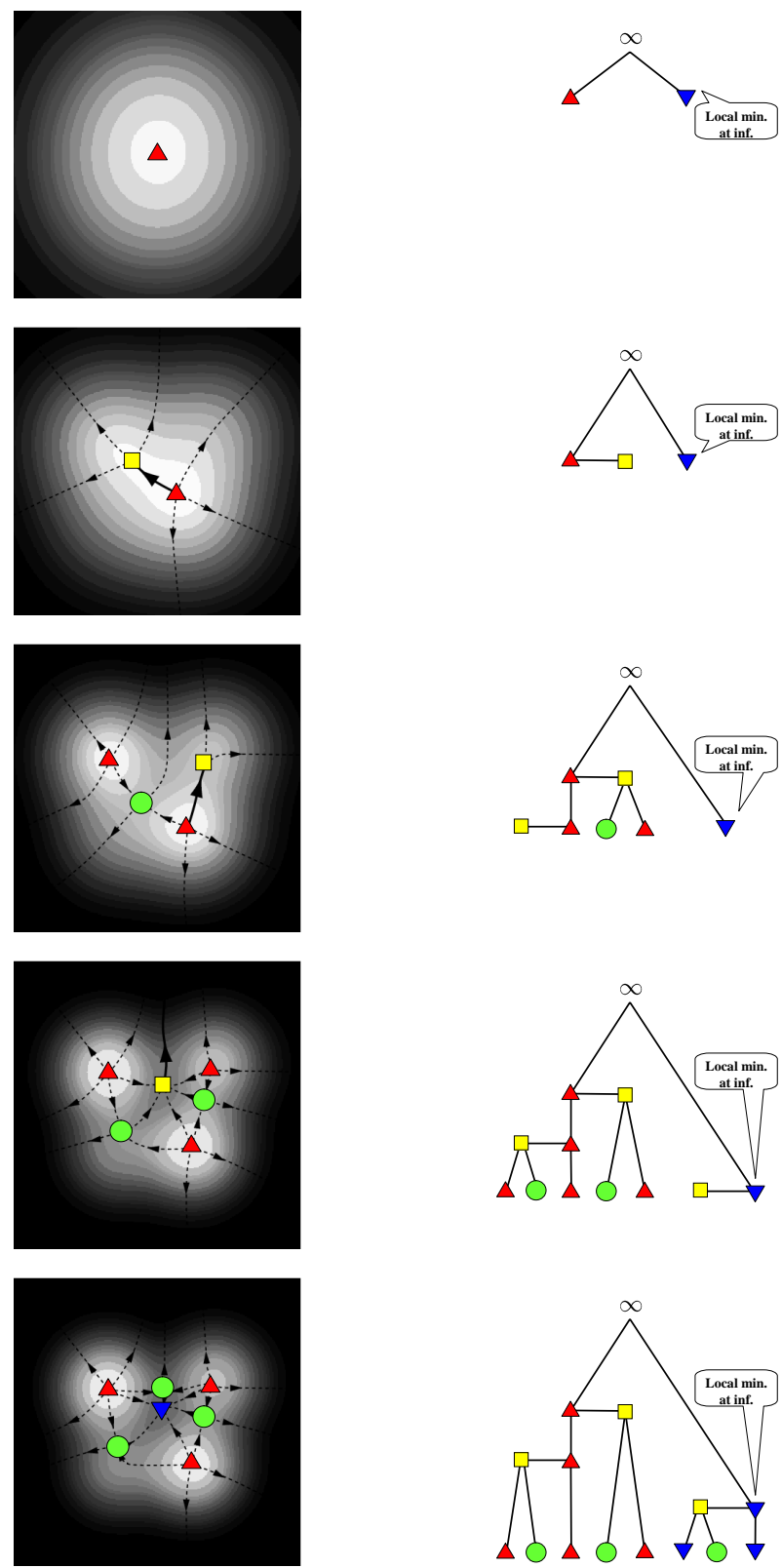

Figure 10: Top-down process of scale-space tree construction. The scale-space images and corresponding trees are displayed in decreasing order of scale from top to bottom. The upward triangles, downward triangles, circles, and squares indicate the local maxima, local minima, saddle points, and annihilation points, respectively. The root of the tree, symbolised by " $\infty$," corresponds to the virtual annihilation point at the infinite scale. 


\subsection{Scale-Space Tree as Qualitative Description of Image}

Whereas an image is a quantitative description of the distribution of intensity values in a space, the tree representation can be considered as a qualitative description of the image. Images with qualitatively the same pattern are described as scale-space trees which indicate the same hierarchical relationships among the stationary points. Conversely, topological difference between trees suggests qualitative disagreement in the image patterns or configurations of the image components represented by the stationary points.

The scale-space tree analysis detects only the topological difference in the configuration of the image components or structure of the image. According to the scale-space axioms, the scale-space tree is invariant under illumination, magnification/contraction, translation, and rotation of the image. These operations on the image do not affect the hierarchical structure.

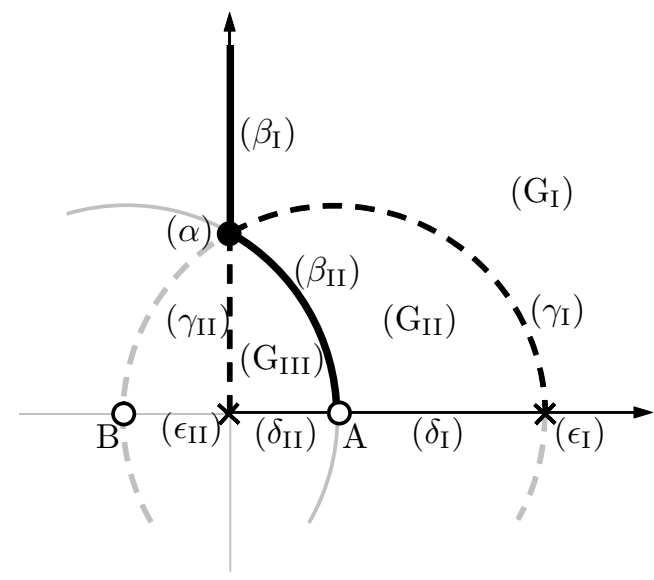

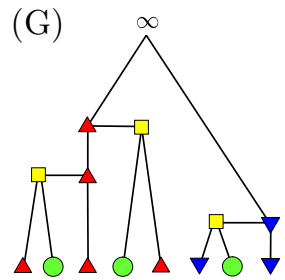

I: $\mathrm{A}$ ab $\mathrm{B}$ ca $\mathrm{C} f$ bc Inf. II: A ca $\mathrm{C}$ ab B $\mathrm{f}$ bc Inf. III: $\mathrm{C}$ ca $\mathrm{A}$ bc $\mathrm{B}$ f ab Inf

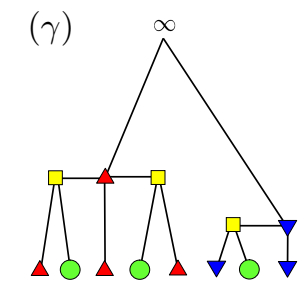

I: $\mathrm{B}$ ab A ca $\mathrm{C}$ f bc Inf. II: $B$ bc $C$ ca $A$ f $a b$ Inf. $(\alpha)$

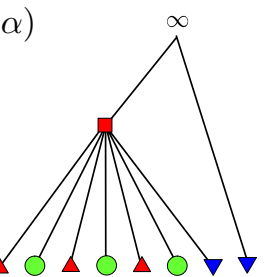

$\mathrm{A}$ ab B bc C ca f Inf.

$(\delta)$

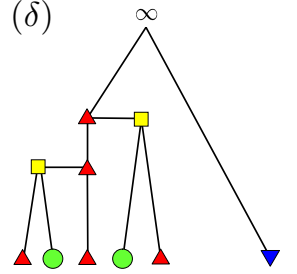

I: $\mathrm{C}$ ca $\mathrm{A}$ ab $\mathrm{B}$ II: A ca $\mathrm{C}$ bc B $(\beta)$

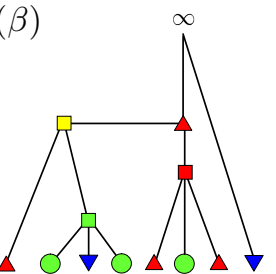

I: $\mathrm{C}$ ca $\mathrm{f}$ bc $\mathrm{A}$ ab $\mathrm{B}$ Inf. II: $B a b$ f bc A ca C Inf.

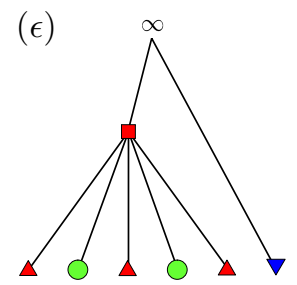

I: $\mathrm{B}$ ab A ca C Inf.

Figure 11: Classification of positions of three bright dots according to the hierarchical relationships among them. The two dots $\mathrm{A}$ and $\mathrm{B}$ are set to be at the points $(-1,0)$ and $(0,1)$. The hierarchy depends on the region where the third $\operatorname{dot} \mathrm{C}$ is located. The nodes $\mathrm{A}, \mathrm{B}$, and $\mathrm{C}$ of the scale-space trees are the local maxima corresponding to the dots. "ab", "bc", and "ca" represent the saddles between the pair of local maxima. "f" indicates the local minimum surrounded by the three local maxima.

Figure 11 shows a simple example of classification by the hierarchical relationships among three dots. Suppose three bright dots A, B, and C with the same intensity are present in a two-dimensional image. We can set $\mathrm{A}$ and $\mathrm{B}$ to be at the points $(1,0)$ and $(-1,0)$ by similarity transformation, translation, and rotation. Distinct types of scale-space tree for the hierarchy of the dots are found 
depending on the position of $\mathrm{C}$. If $\mathrm{P}$ is in the region $G_{\mathrm{II}}$, for example, we obtain the scale-space tree of type G with leaves II. Consequently, we obtain six types of scale-space tree. The generic type is $G$, which indicates that the bright dots are positioned at vertices of a scalene triangle. The other types are nongeneric: $\alpha$ is for the equilateral triangle, and $\beta$ and $\gamma$ are for the isosceles triangle with long and short equal sides, respectively. The three dots are aligned if $\mathrm{C}$ is on $\delta$. They are evenly aligned if $\mathrm{C}$ is at $\epsilon$.

As shown in Fig. 11, relative positions of elements of the image are qualitatively described as the hierarchical tree. If $\mathrm{C}$ moves across regions, the tree structure changes successively. The topological changes of objects in the image or qualitative variations of the image are detectable through the structural changes in a sequence of scale-space trees.

\section{Scale-Space Properties of Figure-Flow Curves}

In this section, we observe the evolution of the figure-flow curves of a two-dimensional image with respect to the scale. The figure-flow curves derive a sequence of pseudographs which topologically represent the scale evolution of the structure. We present topological properties of the figure-flow curves by basic graph analysis. We also focus on considerable figure-flow curves for the evolution of the pseudograph. This figure-flow curve also plays an essential role in the hierarchy of the stationary points, which we discuss in later sections.

\subsection{Pseudograph Representation of Two-Dimensional Image}

The local maxima and local minima are starting and end points of the figure-flow curves, respectively. A saddle point has a pair of one of each inward and outward figure-flow curves, which are called separatrices. Because the separatrices connect two sources of the flow curves, the saddle is a symbol of the connection between the sources. Therefore, the local maxima and the figureflow curves incident to saddle points represent the configurations of the positive components of the two-dimensional positive image.

Zhao and Iijima have proposed a graph representation of a two-dimensional image at a fixed scale (Zhao and Iijima, 1985b). The vertices and edges of the graph represent the local maxima and separatrices, respectively. The faces of the graph correspond to the local minima. This graph represents the watershed structure (Griffin and Colchester, 1995; Olsen and Nielsen, 1997).

Since the separatrices may form multiple edges and self-loops, the graph is a pseudograph. The pseudograph is obtained from the figure field of the two-dimensional image by the following algorithm.

\section{ALGORITHM I - Pseudograph Representation}

1. Set local maxima to be the vertices of the graph.

2. Link the vertices with pairs of figure-flow curves from local maxima to saddle points.

3. Remove the local minima and corresponding figure-flow curves to generate faces.

Figure 12 shows an example of the extraction of the pseudograph from the figure field. Note that figure-flow curves connected to the image borders are removed since they correspond to a local minimum at infinity. Unless the image contains a singular point, we can uniquely derive the pseudograph from the two-dimensional image at any scale. The local minimum at infinity can be regarded as a symbol of the dark background of the positive image. Since the scale-space image $f(\boldsymbol{x}, \tau)$ is positive, the point at infinity is a drain of the figure flow from the whole region of the image.

Stereographic projection is an alternative way of treating extended real space. Figure 13 shows examples of the one-to-one correspondence between the space $\overline{\mathbb{R}}^{1}$ or $\overline{\mathbb{R}}^{2}$ and the circle $\mathbb{S}^{1}$ or sphere $\mathbb{S}^{2}$ under the stereographic projection. The North Pole $\mathrm{N}$ is the point corresponding to the point at infinity. We see that any small displacement on $\mathbb{S}^{1}$ or $\mathbb{S}^{2}$ from $\mathrm{N}$ increases the intensity of the signal or image at the corresponding point $\boldsymbol{x}$. As depicted in Fig. 13, for one- and two-dimensional images, the set of all points in $\overline{\mathbb{R}}^{N}$ is homeomorphic to the sphere $\mathbb{S}^{N}$. The Euler characteristic of $\mathbb{S}^{N}$ is 2 if $N$ is even and 0 if odd. Since the pseudograph for a two-dimensional image is planar at any scale, the pseudograph varies preserving its Euler's characteristic,

$$
\chi=V \overline{18}_{18} E+F,
$$



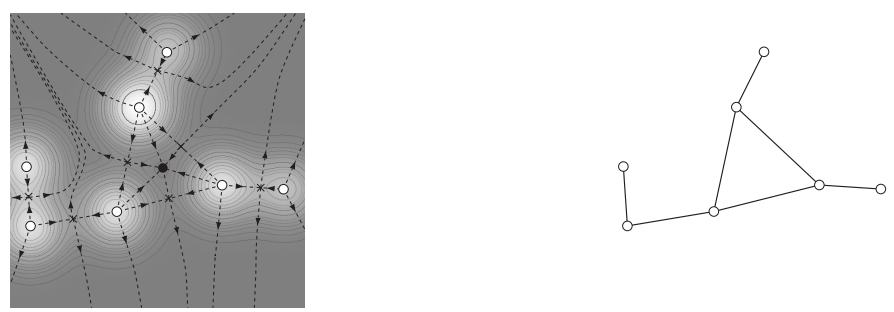

Figure 12: Figure field and corresponding graph.

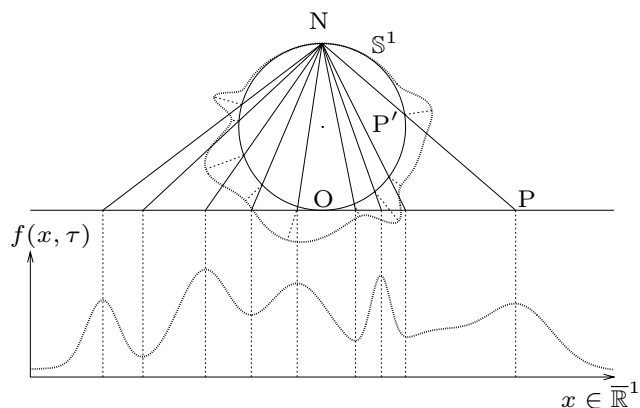

(a)

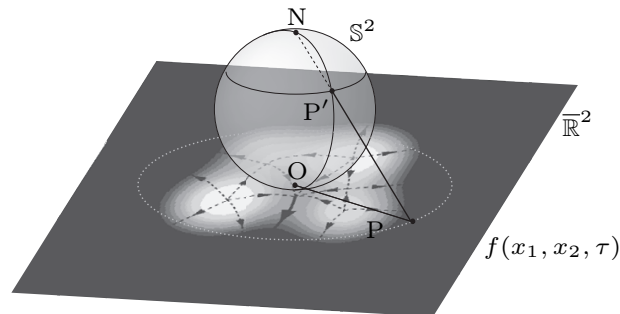

(b)

Figure 13: Stereographic projections of (a) 1D signal $f(x, \tau)$ onto circle, and (b) 2D image $f\left(x_{1}, x_{2}, \tau\right)$ onto Riemann sphere. $\mathrm{N}$ corresponds to the point at infinity, which can be regarded as a local minimum in both cases. These projections are possible for images at any scale $\tau$.
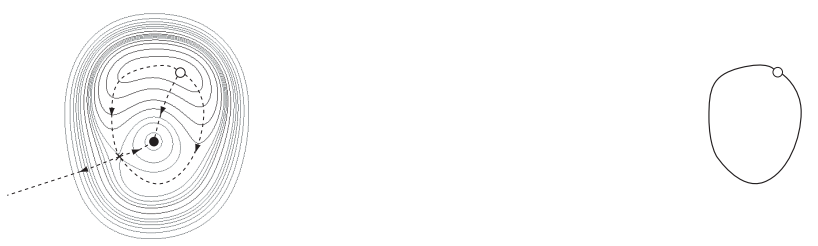

Figure 14: Self-loop.

where $V, E$, and $F$ denote the numbers of vertices, edges, and faces of the pseudograph, respectively. The Euler characteristic $\chi=2$ for the pseudographs. This is a scale-invariant value. The point at infinity corresponds to the exterior face of the pseudograph, so it is counted as one of the $F$ local minima. A face inside a self-loop also represents a local minimum as shown in Fig. 14.

Griffin and Colchester (Griffin and Colchester, 1995) showed the behaviour of the separatrices with respect to scale. They argued that the separatrices, which link stationary points, cannot disappear, shrink, or merge before or after the annihilation and creation of stationary points. They also pointed out a saddle switching catastrophe, which suggests that the connections between the vertices of the pseudograph can change without annihilation or creation. All these variations of pseudograph preserve the Euler characteristic.

According to Eq. (28), an increment (or decrement) in $E$ requires one in $V$ or $F$. This property indicates that creation (or annihilation) of a saddle point is accompanied by that of the local extremum. Shortening an edge to merge two vertices in the pseudograph causes decrements in $E$ and $V$. This process corresponds to the annihilation of a local maximum and a saddle point. Removing an edge to merge two neighbouring faces in the pseudograph results in decrements in $E$ and $F$. This process corresponds to the annihilation of a local minimum and a saddle point. 


\subsection{Experimental Example}

We show an example to illustrate the evolution of the figure-flow curves with respect to the scale. Figure 15 shows 12 snapshots of the diffusion process of a brain image together with the figure-flow curves. The scale-space image at the scale $\tau=10$ has 4 maxima, 2 minima (except the point at infinity) and 5 saddle points.

The evolution of figure-flow curves and corresponding pseudographs with respect to the scale are schematically illustrated in Fig. 16. The stationary points are annihilated with increasing scale. The annihilation events occur approximately at $\tau=19,85,90,95$ and 97 . The figure-flow curves and corresponding pseudographs clarify the connections among stationary points.

This example includes a self-loop in the scale-space image between $\tau=95$ and $\tau=97$. The local minimum point in the self-loop corresponds to a dark internal region of the brain image. The annihilation point of this minimum point is linked to the point at infinity at $\tau=97$. Since the point at infinity is representative of the dark background of the positive image, the link at $\tau=97$ indicates that the dark internal region is derived from the dark background.

\subsection{Antidirectional Figure-Flow Curve}

In Fig. 16, a single figure-flow curve is found ending (starting) at every annihilation point of the local maximum (minimum) and saddle point. This figure-flow curve is indicated by a thick curve at each scale of the annihilation. Except for this curve, the annihilation point of the local maximum (minimum) has outward (inward) curves. Here, we call this considerable figure-flow curve the "antidirectional figure-flow curve". Before the annihilation of an extremum and a saddle, the figureflow curve between these points is shortened. The remaining part of the separatrix becomes the antidirectional figure-flow curve at the annihilation scale.

As can be seen in Fig. 16, the antidirectional figure-flow curve indicates a local extremum to which the annihilated local extremum is subordinate. In the edge shortening and edge removal of the pseudograph, the mergee vertex and face correspond to the annihilated local maximum and minimum, respectively. The merger vertex and face are identified as the local maximum and minimum which is connected to the annihilated maximum and minimum by the antidirectional figure-flow curve.

\section{Combinatorial Structure of Scale-Space Singular Points}

\subsection{Topological Property of Scale Space}

Definition 12 Zhao and Iijima $(1985 a, b)$ For $S(\boldsymbol{x}, \tau)=\left|\frac{d \boldsymbol{x}(\tau)}{d \tau}\right|$, the stationary points on the stationary curves are the points which satisfy $S(\boldsymbol{x}, \tau)=0$ or are isolated points under the conditions $\frac{d S(\boldsymbol{x}, \tau)}{d \tau}=0$ and $\frac{d^{2} S(\boldsymbol{x}, \tau)}{d^{2} \tau}=0$.

Denoting a stationary point on the stationary curves as $\left(\boldsymbol{x}_{i}, \tau_{i}\right)$, the region

$$
\boldsymbol{R}\left(\boldsymbol{x}_{i}, \tau_{i}\right)=\left\{\boldsymbol{x}|| \boldsymbol{x}-\boldsymbol{x}_{i} \mid \leq \sqrt{2 \tau_{i}}\right\}
$$

expresses the dominant part of $f\left(\boldsymbol{x}, \tau_{i}\right)$ as a circular region centred at the point $\boldsymbol{x}_{i}$ with the radius $\sqrt{2 \tau_{i}}$.

Using this monotonically branching curve, we can define the order of scales (Zhao and Iijima, $1985 \mathrm{a}, \mathrm{b})$.

Definition 13 For the stationary points on the stationary curves, the order of the stationary points is defined as $\boldsymbol{x}(\tau) \succ \boldsymbol{x}\left(\tau^{\prime}\right)$ if $\tau>\tau^{\prime}$ on a branch.

In Fig. 17, we show the distribution of stationary points and their view fields. Fig. 17(a) is the original image. Fig. $17(\mathrm{~b})$ is the fields of views of the saddle points in the linear scale space. Fig. $17(\mathrm{c})$ is the fields of views of all stationary points in the scale space. Fig. 17(d) is the field of view of the point whose scale parameter is infinity. Fig. 18 shows scale space analysis of a real image. Fig. 17(a) is the original image, Fig. 17(b) is the stationary curve. Fig. 17(c) is the view fields and stationary points. Fig. $17(\mathrm{~d})$ is the stationary points along the stationary curves in the scale space. As we can observe from these results, that saddle points indicate the regions in which the topology collapses happen as the scale parameter increase 20 


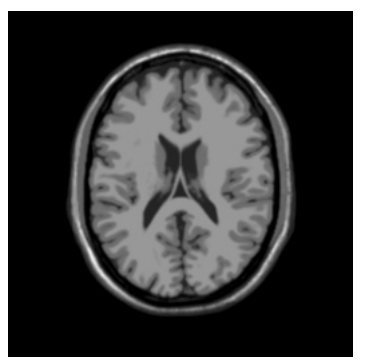

$\tau=0$

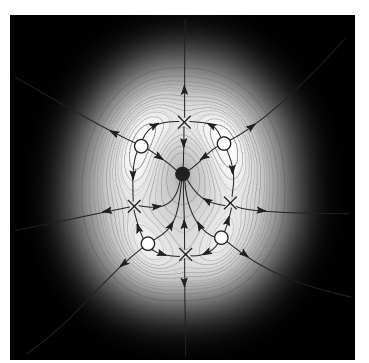

$\tau=30$

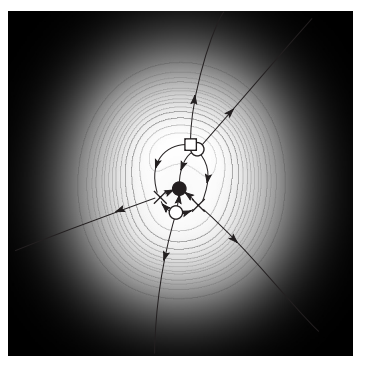

$\tau=89$

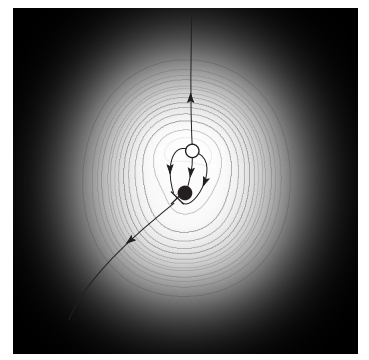

$\tau=96$

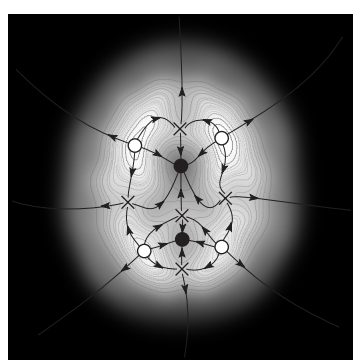

$\tau=10$

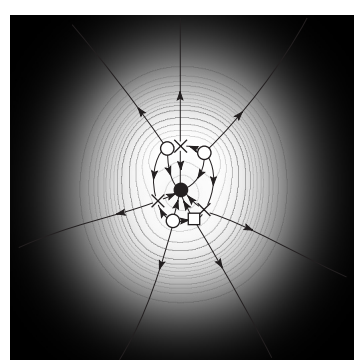

$\tau=85$

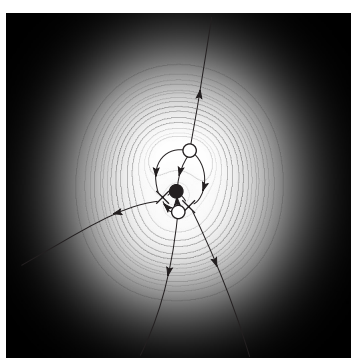

$\tau=93$

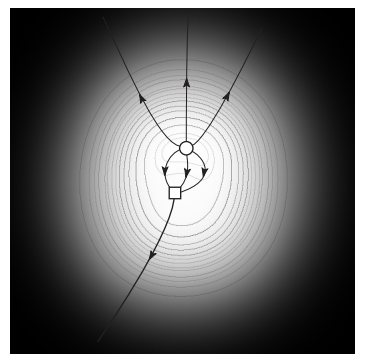

$\tau=97$

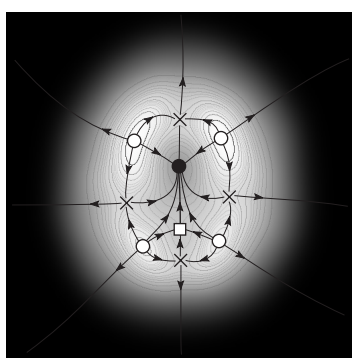

$\tau=19$

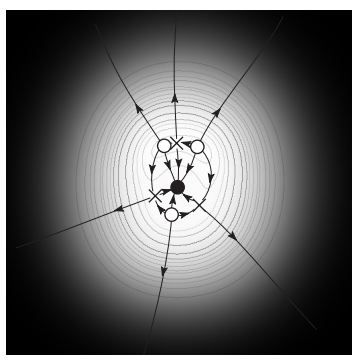

$\tau=87$

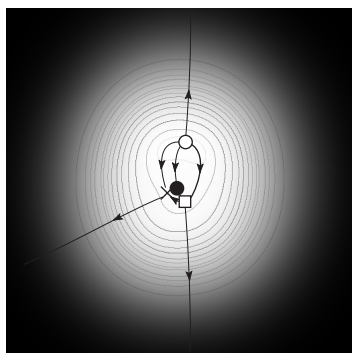

$\tau=95$

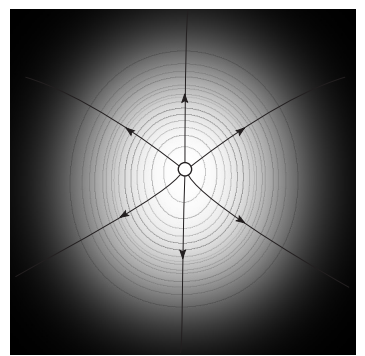

$\tau=125$

Figure 15: Scale-space images of $256 \times 256 \mathrm{MRI}$ of brain, together with schematic drawings of figure-flow curves. Top left figure shows the initial image. Scale increases from left to right, top to bottom. Maxima, minima and saddle points are indicated with open circles, filled circles and crosses, respectively. Annihilation points are plotted with open squares. 

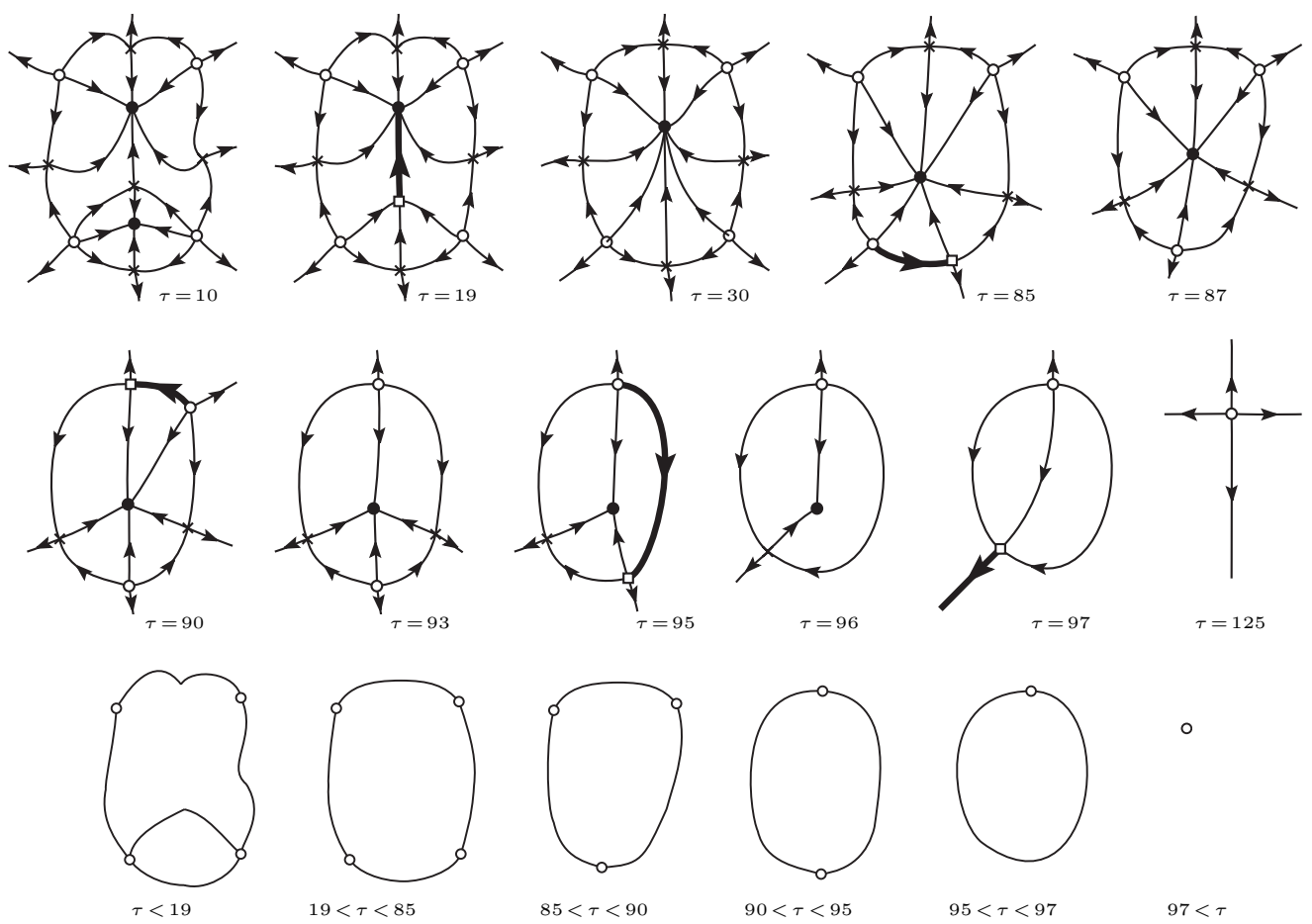

$85<\tau<90$

$90<\tau<95$

$95<\tau<97$

$97<\tau$

Figure 16: Figure-flow curves and pseudographs of MRI brain images. The first and second rows show the evolution of figure-flow curves in increasing order of the scale. Annihilation points are indicated with squares. The third row is a series of pseudographs.

\subsection{Scale-Space Tree in 1D Gaussian Scale Space}

On the extended real line $\overline{\mathbb{R}}$, the generalised function is the solution of the linear diffusion equation

$$
\frac{\partial f}{\partial \tau}=\frac{\partial^{2}}{\partial x^{2}} .
$$

Denoting the signs of the second derivative of points on which the first derivatives are zero as ,+ 0 , and - for $f_{x x}>0, f_{x x}=0$, and $f_{x x}<0$, respectively, these points are the local maximum points, the saddle points, and the local minimum points, respectively. Furthermore, we deal with the point at infinity as local minimum. Now, we define the function $S(x, \tau)$

$$
S(x, \tau)=\left\{\begin{array}{lll}
M, & f_{x}=0, & f_{x x}<0 \\
m, & f_{x}=0, & f_{x x}>0 \\
s, & f_{x}=0, & f_{x x}=0 \\
m_{\infty}, & |x|=\infty &
\end{array}\right.
$$

Using this coding function, for a fixed $\tau$, it is possible to transform $f(x, \tau)$ as a string of $M, m, s$, and $m_{\infty}$ as

$$
S(x, \tau): f(x, \tau)=m_{\infty} s^{*} M s^{*} m s^{*} M s^{*} \cdots M s^{*},
$$

where $s^{*}$ is a string of an appropriate number of $s$. For this string, we have the relation.

Theorem 1 Setting $|M|,|m|$, and $\left|m_{\infty}\right|$ to be the numbers of symbols $m, M$, and $m_{\infty}$ in a string, for $\chi_{1}$,

$$
\chi_{1}=|M|-\left(|m|+\left|m_{\infty}\right|\right),
$$

the relations $\chi=0$ is satisfied for $0 \leq \tau \leq \infty$.

This theorem implies that, for $\tau_{1}<\tau_{2}$, the local transition rule for strings is one of

$$
\begin{aligned}
& \left(M s^{*} m s^{*} M\right)^{*} \rightarrow M \\
& \left(M s^{*} m s^{*} M\right)_{22}^{* 1} \rightarrow\left(M s^{*} m s^{*} M\right)^{* 2},
\end{aligned}
$$




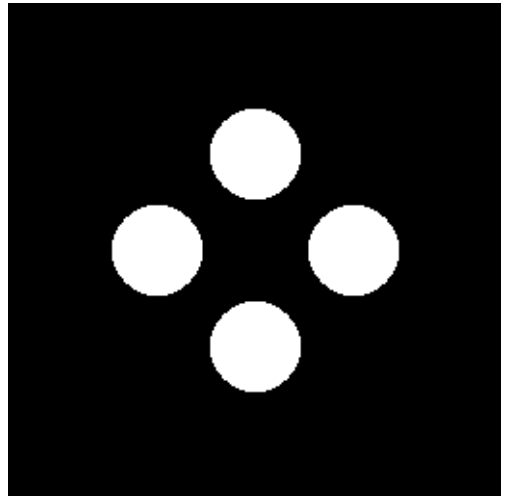

(a)

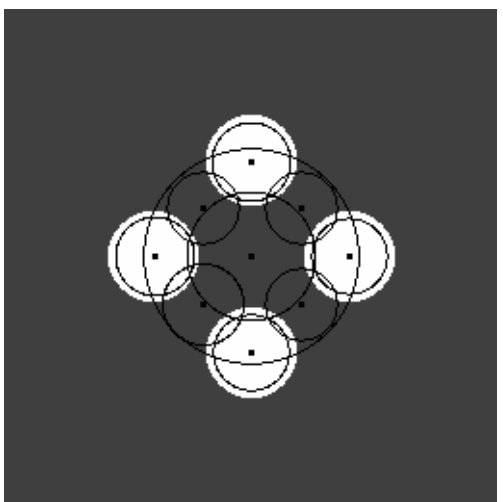

(c)

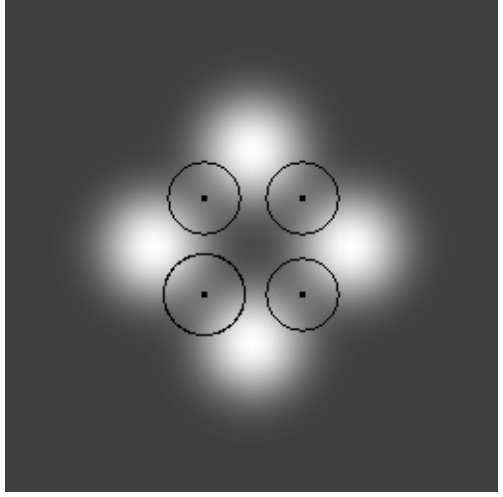

(b)

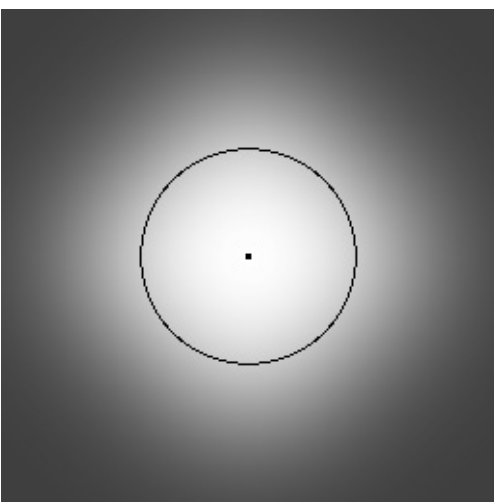

(d)

Figure 17: Scale space analysis of a geometrical synthetic image. (a) original image, (b) view fields of the saddle points in the linear scale space, (c) view fields of all stationary points in scale space, and $(d)$ attention field of the point with a scale parameter of infinity.

for $\left[\left(M s^{*} m s^{*} M\right)^{* 1}\right]>\left[\left(M s^{*} m s^{*} M\right)^{* 2}\right]$, where $[a]$ is the length of the string $a$. These rules derive the string

$$
\left(M s^{*} s^{*} M\right)^{*} m_{\infty} \rightarrow^{*} M m_{\infty}
$$

after the successive application of rewriting rules more than one time. Then, finally we have the rule

$$
M m_{\infty} \rightarrow \emptyset
$$

The local rule of Eq. (34) is separated into two steps

$$
M m M \rightarrow M s \rightarrow M, M m M \rightarrow s M \rightarrow M
$$

for the configuration of $M m M$. In this process, a maximal curve which expresses the intermediate configurations $s M$ and $M s$ are locally the main trunk. This process is described that a pair of local maximum and minimum curves which are merged to the local main trunk after they are merged at the point both the first and second derivatives are zero. This is the combinatorial re-statement of the Zhao and Iijima (Zhao and Iijima, 1985a) rules for the merging of stationary curves in the Gaussian scale space.

This combinatorial structure is equivalent to the transition of the topology of a function with respect to the scale parameter $\tau$ on $x-\tau-f(x, \tau)$ space. The branching geometry and the location of point $\left(x_{s}, \tau\right)^{\top}$, where $f_{x}\left(x_{s}\right)=0$ and $f_{x x}\left(x_{s}\right)=0$, completely and uniquely define the structure of tree in the Gaussian scale space.

The intermediate configurations $M s$ and $s M$ express that $m M$ and $M m$ are merged to $s$, on which both the first and second derivatives are zero and that after merging two singular points a 


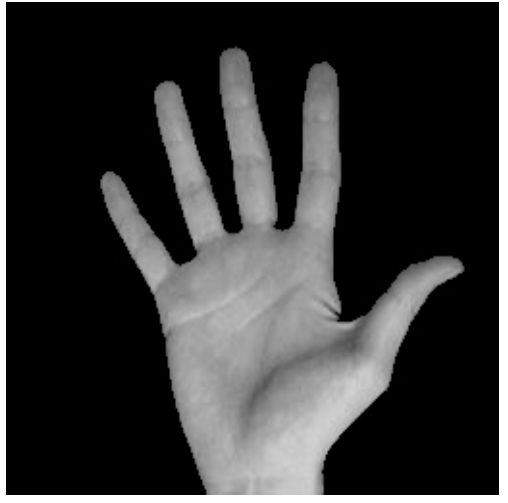

(a)

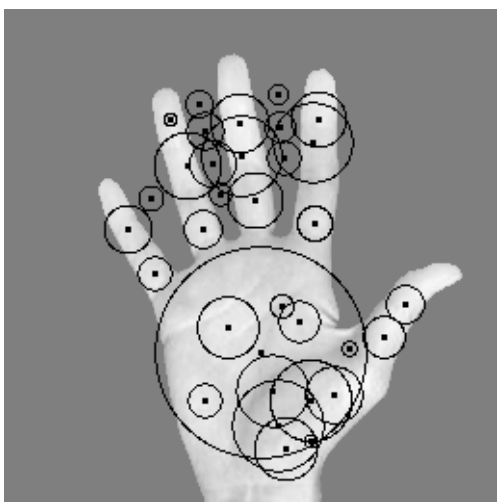

(c)

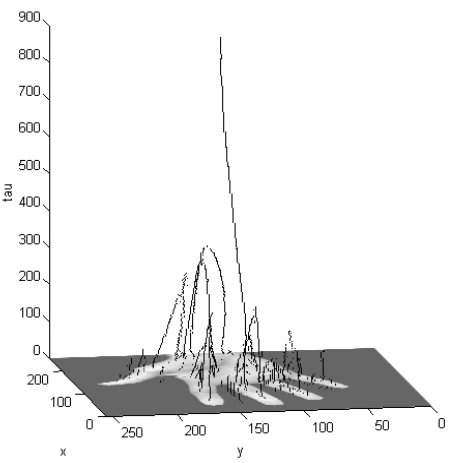

(b)

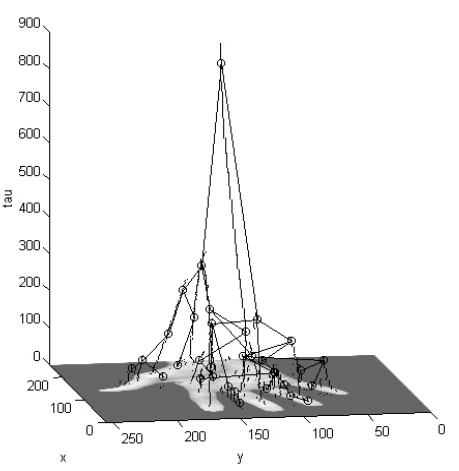

(d)

Figure 18: Scale space analysis of an image. (a) original image, (b) stationary curve, (b) view fields and stationary points, and (c) stationary points along the stationary curves in the scale space.

local maximum survives. If both rules are applied concurrently, we have the third local rule,

$$
M m M \rightarrow s m s \rightarrow M .
$$

These processes derive binary branching structures as

$$
\langle M, s\langle m, M\rangle\rangle, \quad\langle\langle M, m\rangle s, M\rangle
$$

and trinary branching structure

$$
\langle M, s\langle M, m, M\rangle\rangle .
$$

Using these structures, it is possible to construct a tree from the trajectory of singular points in the Gaussian scale space. The generalisation of these symbol-transition rules define the following two branching structures

$$
\begin{aligned}
& \langle M\langle s\langle M, m, M, m, \cdots, m, M\rangle\rangle \\
& M\langle M, s\langle M, m\rangle, s\langle M, m\rangle, \cdots, s\langle M, m\rangle\rangle\rangle
\end{aligned}
$$

In the first structure the numbers of $m$ and $M$ are $(k+1)$ and $(k+2)$ for $k \geq 0$. In the second structure the number of the primitive $s\langle M m\rangle$ is $k \geq 1$. A special case of the first structure is

$$
\langle M, s\langle m, M\rangle\rangle,\langle\langle M, m\rangle s, M\rangle
$$

which is a primitive of the branching structures.

Since there is one-to-one mapping between $\overline{\mathbb{R}}$ and the unit circle $\mathbb{S}$, it is possible to transform the string of singular-point symbols to vertices of a polygon whose vertices lie on the unit circle. We 
transform symbols $M$ and $m$ to the labels on the vertices and edges of this polygon. This relation is shown in Figure 1. Since the merging rule $M m M \rightarrow M$ corresponds to the elimination of an edge and a vertex, the transition of the configuration of singular points corresponds to the simplification of polygon. In this simplification process, finally a vertex and an loop are merged to empty.

We show a simple example of scale-space singular-point evolution and tree construction from this evolution. The evolution of strings is described as

$$
M_{1} m M_{2} m_{\infty} \rightarrow M_{1} s m_{\infty} \rightarrow M_{12} m_{\infty} \rightarrow \emptyset .
$$

This evolution of strings corresponds to the evolution of function graphs in the Gaussian scale space. This evolution of function derives the tree

$$
\emptyset\left\langle m_{\infty}, M_{12}\left\langle M_{1}, s\left\langle m, M_{2}\right\rangle\right\rangle\right\rangle .
$$

\subsection{Scale-Space Tree in 2D Gaussian Scale Space}

Denoting the signs of the eigenvalues of the Hessian matrix of a two-dimensional function $f$ as $(-,-),(+,-)$ and $(+,+)$ in the Gaussian scale space, these labels of points correspond to the local maximum points, the saddle points, and the local minimum points, respectively. For twodimensional positive functions with a finite number of extrema, we define the labelling function such that

$$
S(\boldsymbol{x}, \tau)=\left\{\begin{array}{lll}
M M, & r=2, & \lambda_{i}<0, \\
M m, & r=2, & \lambda_{1} \cdot \lambda_{2}<0, \\
m m, & r=2, & \lambda_{i}>0, \\
s M, & r=1, & \lambda_{1}<0 \\
s m, & r=1, & \lambda_{1}>0, \\
m_{\infty} & |\boldsymbol{x}|=\infty, &
\end{array}\right.
$$

for points $\nabla f=0$ for the eigenvalues of the matrix $\boldsymbol{H} . \quad s M$ and $s m$ correspond to $s$ in $1 \mathrm{D}$ configurations.

Since $M s$ and $m s$ correspond to $s$ in 1D configurations, these configurations appear as $(M M)(M s)^{*}(M m)$ and $(m m)(m s)^{*}(m M)$ in 2D configurations. For these two configurations, we have local rewriting rules,

$$
\begin{aligned}
& (M M)(M m)(M M)=M(M m M) \rightarrow M M, \\
& (M m)(m m)(M m)=(M m M) m \rightarrow M m, \\
& (m m)(M m)(m m)=(m M m) m \rightarrow m m .
\end{aligned}
$$

Using these rules, we have a simple example of transition such that

$$
\begin{aligned}
& M M \quad M M \\
& M m \quad m m \quad m M \rightarrow M m \rightarrow \cdots \rightarrow M M \rightarrow M M m_{\infty} \rightarrow \emptyset . \\
& M M \quad m M \quad M M \quad M M
\end{aligned}
$$

The intermediate configurations

$$
\begin{aligned}
& M(M m M) \rightarrow M(s M M) \rightarrow M M, \\
& (M m M) M \rightarrow(M s M) M \rightarrow M M, \\
& m(M m M) \rightarrow m(s M M) \rightarrow M m, \\
& (M m M) m \rightarrow(M s M) m \rightarrow M m, \\
& m(m M m) \rightarrow m(s m m) \rightarrow m m, \\
& (m M m) m \rightarrow(m s m) m \rightarrow m m,
\end{aligned}
$$

define the order of the hierarchical expression, the same as in the case of 1D functions. If all three transitions appear concurrently, the tree

$$
M M\langle M M, M m, M M, M m, m m, M m, M M\rangle
$$

is derived. This transition appears if three isotropic Gaussians with the same variance are located at the three vertices of a regular triangle. For any triangle, the tree structures are derived as rotations of the tree

$$
M M\left\langle M M, s M\left\langle m M\left\langle m M, s m\left\langle m m_{25} m M\right\rangle M M\langle M M, s M\langle m M, M M\rangle\rangle\right\rangle .\right.\right.
$$


Figure 19 shows trees of triangle probes: (c) is the tree extracted from a regular triangle. (d) is the tree extracted from a triangle. Figure 20 shows the configurations of singular points for triangles.

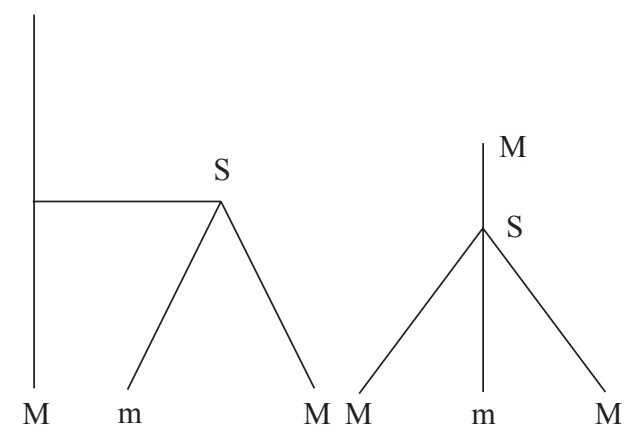

(a)

(b)

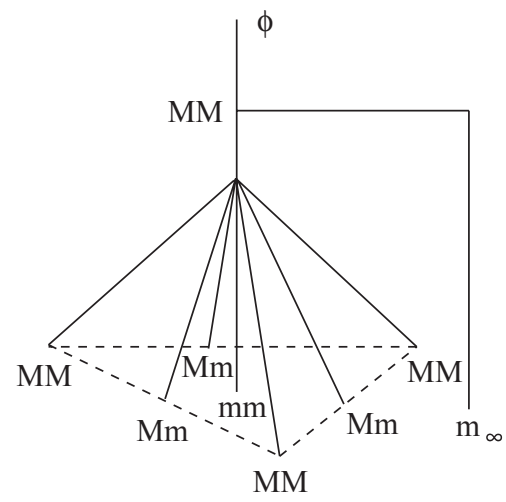

(c)

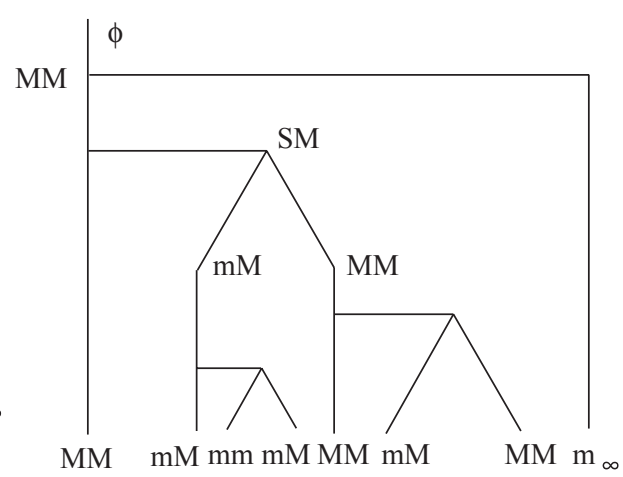

(d)

Figure 19: Trees of triangle probes. (a) and (b) are two primitive branch structures for a 1D function. (c) The tree extracted from a regular triangle. (d) The tree extracted from a triangle

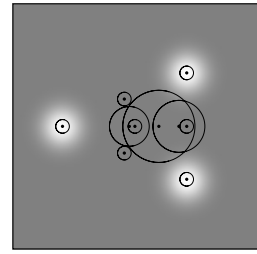

(a)

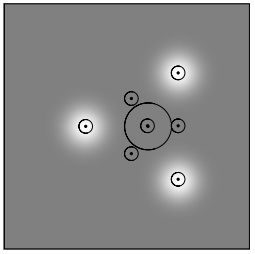

(b)

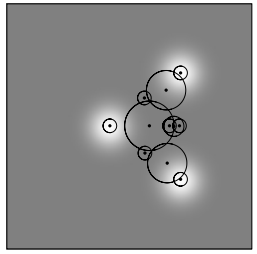

(c)

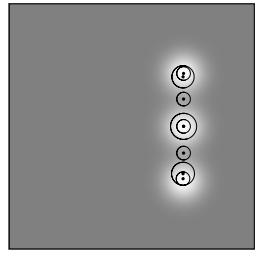

(d)

Figure 20: Singular point configuration of triangles. (a), (b), (c), and (d) show a sequenc of images from left to right. A peak of a Gaussain moves from left to center of the other two peaks.

These two tree structures are the primitives of tree structures derived by Gaussian-scale-space singular-point analysis. Therefore, combinations of these two primitives and three rewiring rules describe the transition of the configurations and derive tree structures from branching structures. This combinatorial structure is equivalent to the transition of the topology of a function with respect to the scale parameter $\tau$ on $\boldsymbol{x}-\tau-f(\boldsymbol{x}, \tau)$ space. The branching geometry and the location of point $\left(\boldsymbol{x}_{s *}, \tau\right)^{\top}$, where $\nabla f\left(\boldsymbol{x}_{s *}\right)=0$ and $\operatorname{rank} \nabla \nabla^{\top} f\left(\boldsymbol{x}_{s *}\right)=1$, completely and uniquely define the structure of the tree in the Gaussian scale space. 


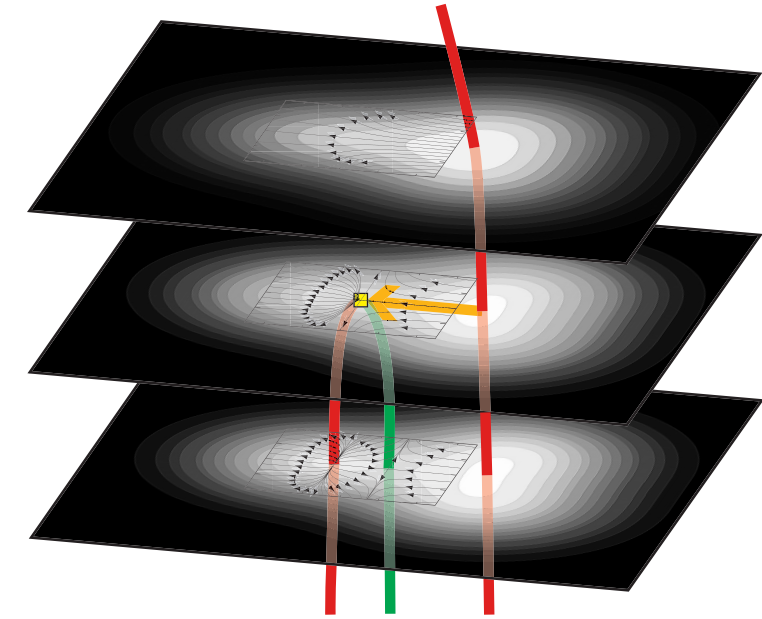

(a)

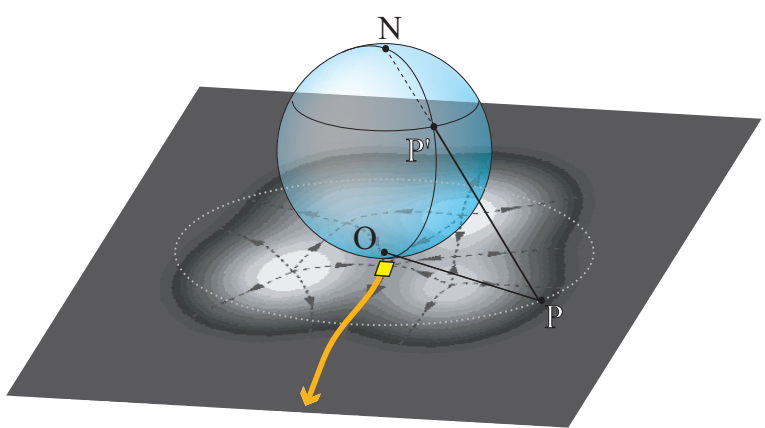

(b)

Figure 21: Evolution of a function in the linear scale space. (a) Merging process of stationary curves for a two-dimensional function. (b) The spherical expression of the singular-point configuration.

Figure 21 shows the evolution of a function in the Gaussian scale space. Fig. 21(a) is merging process of stationary curves for a two-dimensional function and (b) is the spherical expression of the singular-point configuration. According to one-to-one mapping between a function on Euclidean plane $\overline{\mathbb{R}}^{2}$ and the unit sphere $\mathbb{S}^{2}$, the scale space extrema, local maxima, saddle, and local minima correspond to vertices, edges, and faces on a polyhedron. Furthermore, these extrema are defined as the results of Voronoi tessellation and Delaunay triangulation in the following manner.

1. Construct Voronoi tessellation form generators such that,

$$
\left.\nabla f\right|_{\boldsymbol{x}}=0, \boldsymbol{H} \boldsymbol{x}=\lambda \boldsymbol{x}
$$

for $\lambda>0$.

2. Construct Delaunay triangulation from Voronoi tessellation.

3. Accept generators as local maxima, which become Delaunay vertices.

4. Accept the common point between Voronoi edges and Delaunay edges as saddles.

5. Accept Voronoi vertices as local minima.

6. Accept the infinite point as a local minimum.

Since a Delaunay graph of a finite number of generators on a plane is a finite graph, this Delaunay graph derives a polyhedron which is topologically equivalent to the unit-sphere in the threedimensional Euclidean space. In this transformation, the infinite face which is the infinite region separated by the boundary edges of the graph corresponds to the minimum at infinity.

Same as the scale-space evolution of one-dimensional functions, the evolution of the singularpoint configurations in the linear scale space of a two-dimensional function is the evolution of a polyhedral graph through the elimination of vertices.

Using one-to-one correspondences between a sphere and the Euclidean plane, for the numbers of the singular points, we have the next theorem.

Theorem 2 Setting $|M M|, M m|| m m \mid$, , and $\left|m_{\infty}\right|$ to be the numbers of singular points with symbols $M M, M m, m m$, and $m_{\infty}$, for $\chi_{2}$,

$$
\chi_{2}=|M M|-|M m|+\left(|m m|+\left|m_{\infty}\right|\right)
$$

the relation $\chi_{2}=2$ is satisfied for $0 \leq \tau \leq \infty$. 


\subsection{Scale-Space Tree in 3D}

Symbolically, it is possible to extend the grammars proposed in the previous sections to 3D objects.

Denoting the signs of the eigenvalues of the Hessian matrix of a three-dimensional function $f$ as $(-,-,-),(+,-,-),(+,+,-)$, and $(+,+,+)$ in the linear scale space, these labels of points correspond to the local maximum points, the negative saddle points, the positive saddle points and the local minimum points, respectively.

For three-dimensional positive functions with a finite number of extrema, we define a labelling function such that

$$
S(\boldsymbol{x}, \tau)=\left\{\begin{array}{lll}
M M M, & r=3, & \lambda_{i}<0, \\
M M m, & r=3, & \lambda_{1}>0>\lambda_{2} \geq \lambda_{3}, \\
M m m, & r=3, & \lambda_{1} \geq \lambda_{2}>0>\lambda_{3}, \\
m m m, & r=3 & \lambda_{i}>0, \\
s M M, & r=2, & \lambda_{i}<0, \\
s M m, & r=2, & \lambda_{1}>0>\lambda_{2}, \\
s m m, & r=2, & \lambda_{1} \geq \lambda_{2}>0, \\
s s M, & r=1, & \lambda_{1}<0, \\
s s m, & r=1, & \lambda_{1}>0, \\
m_{\infty}, & |\boldsymbol{x}|=\infty,
\end{array}\right.
$$

for points $\nabla f=0$, where $\lambda_{i} i=1,2,3$ are eigenvalues of $\boldsymbol{H}$ and $r=\operatorname{rank}\left(\nabla \nabla^{\top} f\right)$. Labels $s * *$ and $s s *$ correspond to $s$ in $1 \mathrm{D}$ configurations.

Since labels $s * *$ and $s s *$ correspond to $s$ in $1 \mathrm{D}$ configurations, we have the the rewriting rules.

$$
\begin{aligned}
& (M M M)(M M m)(M M M) \rightarrow M M(M M m) \rightarrow M M M \\
& (M M m)(M m m)(M M m) \rightarrow M m(M M m) \rightarrow M M m \\
& (M m m)(m m m)(M m m) \rightarrow m m(M M m) \rightarrow M m m \\
& (M M m)(M M M)(M M m) \rightarrow M M(M m m) \rightarrow M m m \\
& (M m m)(M M m)(M m m) \rightarrow M m(M M m) \rightarrow M M m \\
& (m m m)(M m m)(m m m) \rightarrow m m(M m m) \rightarrow m m m .
\end{aligned}
$$

For these rules, the intermediate configurations are expressed as

$$
\begin{aligned}
& (M M M)(M M m)(M M M) \rightarrow M M(M M m) \rightarrow M M(s M M m) \\
& (M M m)(M m m)(M M m) \rightarrow M m(M M m) \rightarrow M m(s M M m) \\
& (M m m)(m m m)(M m m) \rightarrow m m(M M m) \rightarrow m m(s M M m) \\
& (M M m)(M M M)(M M m) \rightarrow M M(M m m) \rightarrow M M(s M m m) \\
& (M m m)(M M m)(M m m) \rightarrow M m(M M m) \rightarrow M m(s M m m) \\
& (m m m)(M m m)(m m m) \rightarrow m m(M m m) \rightarrow m m(s M m m) .
\end{aligned}
$$

These intermediate configurations define the order of the branching structure of trees. This combinatorial structure is equivalent to the transition of the topology of a function with respect to the scale parameter $\tau$ on $\boldsymbol{x}-\tau$ - $f(x, \tau)$ space. The branching geometry and the location of point $\left(x_{s * *}, \tau\right)^{\top}$, where $\nabla f\left(\boldsymbol{x}_{s * *}\right)=0$ and $\operatorname{rank} \nabla \nabla^{\top} f\left(\boldsymbol{x}_{s * *}\right)=2$, completely and uniquely define the structure of a tree in the linear scale space.

Using one-to-one mapping from $\overline{\mathbb{R}}^{3}$ to $\overline{\mathbb{S}}^{3}$, for the numbers of singular points, we have the next theorem since singular points with labels $(M M M),(M M m),(M m m)$, and $(m m m)$ correspond to the vertices, edges, faces, and volumes of polytopes in $\overline{\mathbb{R}}^{3}$.

Theorem 3 Setting $|* * *|$ to be the number of singular points with symbol $* * *$,

$$
\chi_{3}=|M M M|-|M M m|+|M m m|-\left(|m m m|+\left|m_{\infty}\right|\right),
$$

the relation $\chi_{3}=0$ is satisfied.

It is possible to deal with 4-dimensional polytopes as spatial graphs in $\overline{\mathbb{R}}^{3}$, the same as the case in which we deal with a polyhedral without any holes as a planar graph. 
It is possible to deal with 4-dimensional polytopes as spatial graphs in $\mathbf{R}^{3}$, same as the case that we deal with a polyhedral without any holes as a planar graph. For example, if the singular points are located on a polyhedral graph in $\mathbb{R}^{3}$ shown in Fig. 22(a). we have the relation $|M M M|=8$, $|M M m|=14,|M m m|=9,|m m m|=2$, and $\left|m_{\infty}\right|=1$. Furthermore, the graphical transition of configurations of singular points are also described in Fig. 22. Then we have the transition of the numbers of singular points listed in Table 1.

Table 1: Numbers of Singular Points in Scale Space

$\begin{array}{ccccccc}\text { Fig. } & |M M M| & |M M m| & |M m m| & |m m m| & \left|m_{\infty}\right| & \chi_{3} \\ & |M M| & |M m| & & |m m| & & \chi_{2} \\ & |M| & & & |m| & & \chi_{1} \\ \text { (a) } & 8 & 14 & 9 & 2 & 1 & 0 \\ \text { (b) } & 6 & 12 & 9 & 2 & 1 & 0 \\ \text { (c) } & 4 & 4 & & 1 & 1 & 2 \\ \text { (d) } & 2 & & & 1 & 1 & 0 \\ \text { (e) } & 1 & & & & 1 & 0\end{array}$

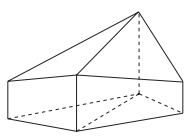

(a)

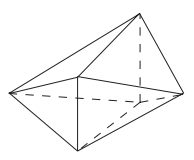

(b)

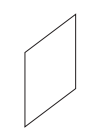

(c)

(d)

(e)

Figure 22: Transition of a polyhedral graph. In (a) and (b) the configuration of points with label $M M M$ is spatial In (c) after two vertices are eliminated, the configuration of singular points is planar. In (d) and (e), the singular points configurate on a line.

\subsection{Scale Space Tree in ND Gaussian Scale Space}

The signs of the eigenvalues of the Hessian matrix of a function $f$ in $n$-dimensional Euclidean space $\mathbb{R}^{N}$, enables us to classify the local topological structure of the points $\boldsymbol{x}^{*}$ which satisfy $\nabla f\left(\boldsymbol{x}^{*}\right)=0$. Setting the numbers of zero, positive, and negative eigenvalues of the Hessian matrix at the point $\boldsymbol{x} *$ to be $\alpha, \beta, \gamma$, respectively, the rank of the Hessian matrix at the point $\boldsymbol{x}^{*}$ is $r=\beta+\gamma$. As define in section 6.3 , we affix the label $s^{\alpha} M^{\beta} m^{\gamma}$ to this point $\boldsymbol{x}^{*}$. 
For $N$-dimensional positive functions with a finite number of extrema, we define a labelling function such that

$$
S(\boldsymbol{x}, \tau): f(x, \tau)= \begin{cases}s^{\alpha} M^{\beta} m^{\gamma} & \alpha=N-\operatorname{rank} \boldsymbol{H}, \\ & \beta=|\{\lambda>0 \mid \boldsymbol{H} \boldsymbol{u}=\lambda \boldsymbol{u}\}|, \\ & \gamma=|\{\lambda<0 \mid \boldsymbol{H} \boldsymbol{u}=\lambda \boldsymbol{u}\}|, \\ m_{\infty}^{N}, & |\boldsymbol{x}|=\infty,\end{cases}
$$

for points $\nabla f=0$. The label $s *$ correspond to $s$ in $1 \mathrm{D}$ configurations.

Using one-to-one mapping from $\overline{\mathbb{R}}^{N}$ to $\mathbb{S}^{N}$, a point with label $s^{\alpha} M^{\beta} m^{\gamma}$ is corresponds to a $\gamma$ dimensional facet in a $(\beta+\gamma)$-dimensional Euclidean space, that is, the maximum point $M^{N}$ and the minimum point $m^{N}$ correspond to a vertex and a face, respectively.

This correspondence allows us to deal with the configuration of extremal of a function in $\overline{\mathbb{R}}^{N}$ as a spatial graph in $\overline{\mathbb{R}}^{N+1}$. For the numbers of the singular points, we have the next theorem

Theorem 4 Setting $|* * \cdots *|$ to be the number of singular points with symbol $* * \cdots *$,

$$
\sum_{\beta=0}^{\beta+\gamma}\left|s^{\alpha} M^{\beta} m^{\gamma}\right|+(-1)^{\beta+\gamma}=0 .
$$

\subsection{Top-Point Tree}

We call the trees defined in the previous sections the stationary trees. The top points for twovariable functions are the points at which the rank of the Hessian matrix is one. Using singular points, we define the top-point.

Definition 14 On the top points, the rank of the Hessian matrix is less than the number of variables of the functions.

Therefore, a top point corresponds to the branch point of the stationary curves for two-valued functions. This geometric property derives an algorithm for the construction of the top-point tree from the stationary tree. Using the stationary trees, we define the hierarchical structure of the top points. On a stationary tree, a top point correspond to a node labelled $s, s *$, and $s * *$ for $1-, 2-$, and 3 -variable functions, respectively. This geometrical property permits us to mathematically define the top-point tree from the stationary tree.

Theorem 5 By purring leaves without label s from the stationary tree, the top-point tree is constructed.

This definition implies there is one-to-one mapping between a stationary tree and a top point tree for a function. Figure 23 shows the extracted top-point tree from the stationary tree.

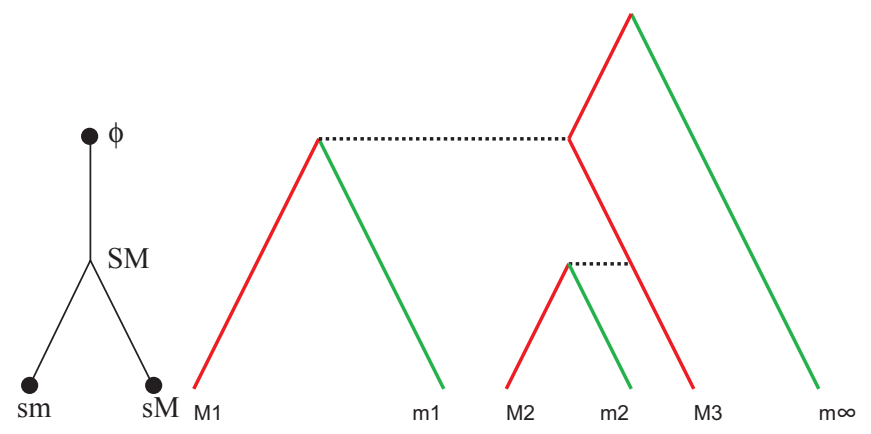

(a)

(b)

Figure 23: Tree and top-point tree. (a) The top-point tree extracted from the tree in (b). (b) Merging process of stationary curves for a two-dimensional function. 


\subsection{Approximate Tree Distance and its Fast Computation}

For quantitative analysis of topological changes of a temporal image with temporal trees extracted in the linear scale space, we introduce the distance between trees on the basis of editing of the tree structure. Since it is possible to transform irregular trees to regular trees by adding special nodes (*) to the trees, we assume that our trees are regular. Furthermore, since our trees, extracted by Gaussian scale space analysis, are rooted trees, we develop a fast computation method for rooted trees which is applicable in time-varying image sequence analysis (Kawashima, Imiya, and Nishida, 1996).

We assume that trees are $m$-regular, for $m \geq 2$. Setting $\alpha$ to be a $k$-digit number, each digit of which is from 0 to $k-1$, the subtree of the node $\alpha$ is expressed as

$$
n_{\alpha}(T)=t_{\alpha}\left[T_{\alpha 1}, T_{\alpha 2}, \cdots, T_{\alpha m}\right],
$$

where $t_{\alpha}$ is the label of node $n_{\alpha}$. For $\alpha=0$, equation (77) expresses the tree and $n_{0}$ is the label of the root. Therefore, the number of digits of $\alpha$ expresses the depth of a subtree. The operations applied to a tree are the transform of the node label, the permutation of subtrees, the insertion of a subtree to $*$, and the elimination of a subtree. These operations are mathematically expressed as

$$
\begin{aligned}
E_{t}: t_{\alpha}\left[T_{\alpha 1}, \cdots, T_{\alpha m}\right] & =t_{\alpha}\left[T_{\alpha 1}, \cdots, T_{\alpha m}\right], \\
E_{p}: t_{\alpha}\left[T_{\alpha 1}, \cdots, T_{\alpha i} \cdots T_{\alpha j} \cdots T_{\alpha m}\right] & =t_{\alpha}\left[T_{\alpha 1}, \cdots, T_{\alpha j} \cdots T_{\alpha i} \cdots T_{\alpha m}\right], \\
E_{i}(S): t_{\alpha}\left[T_{\alpha 1} \cdots * \cdots T_{\alpha m}\right] & =t_{\alpha}\left[T_{\alpha 1} \cdots S \cdots T_{\alpha m}\right], \\
E_{i}\left(T_{\alpha k}\right): t_{\alpha}\left[T_{\alpha 1} \cdots T_{\alpha k} \cdots T_{\alpha m}\right] & =t_{\alpha}\left[T_{\alpha 1} \cdots * \cdots T_{\alpha m}\right] .
\end{aligned}
$$

Furthermore, a successive application of $E_{n}$ derives the transformation of a subtree such as

$$
E_{t}\left(T_{\alpha, k}, S\right): t_{\alpha}\left[T_{\alpha 1}, \cdots, T_{\alpha k} \cdots, T_{\alpha m}\right]=t_{\alpha}\left[T_{\alpha 1}, \cdots, S, \cdots, T_{\alpha m}\right] .
$$

We define the lengths of these operations as

$$
d\left(s_{\alpha}\right)=\frac{1}{1+l_{k}}\left|s_{\alpha}\right|,
$$

where

$$
l_{\alpha}= \begin{cases}0, & \text { if } \alpha=0, \\ \text { the number of digits of } \alpha, & \text { otherwise }\end{cases}
$$

and

$$
\left|s_{\alpha}\right|= \begin{cases}1, & \text { if } s_{\alpha} \text { is the node-label transform, } \\ |P|, & \text { if } s_{\alpha} \text { is the permutation, } \\ |S|, & \text { if } s_{\alpha} \text { is the insertion, } \\ |S|, & \text { if } s_{\alpha} \text { is the elimination, }\end{cases}
$$

where $|P|$ is the number of permutations. Using these lengths, we define the distance between trees as

$$
D\left(T, T^{\prime}\right)=\sum_{\alpha=1}^{n} d\left(s_{\alpha}\right),
$$

for the sequence of operations $\left\{s_{1}, s_{2}, \cdots, s_{n}\right\}$ which transforms $T$ to $T^{\prime}$. This tree distance satisfies the following lemma.

Lemma 1 For trees of almost the same order, the distance in Eq. (86) is metric, that is, it satisfies the conditions of distance.

Proof 2 It is obvious that $D(T, T)=0$ and that $D\left(T, T^{\prime}\right)=D\left(T^{\prime}, T\right)$. Setting

$$
\left\{s_{1}, s_{2}, \cdots, s_{n}\right\},\left\{t_{1}, t_{2}, \cdots, t_{n}\right\},\left\{u_{1}, u_{2}, \cdots, u_{n}\right\}
$$

to be the sequences of transformation from $T$ to $T^{\prime}$ and $T^{\prime}$ to $T^{\prime \prime}$, for a triplet of trees of almost the same size, we obtain the relation

$$
\left|s_{k}\right|+\left|t_{k}\right| \geq\left|u_{k}\right|
$$

by considering a non-operation which satisfies

$$
\mid \text { operation } \mid=0 \text {. }
$$

This relation derives the relation

$$
D\left(T, T^{\prime}\right)+D\left(T_{31}^{\prime}, T^{\prime \prime}\right) \geq D\left(T, T^{\prime \prime}\right) .
$$




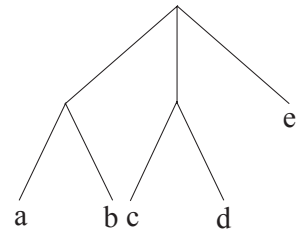

(a)

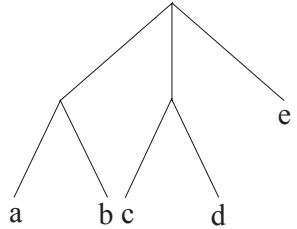

(b)

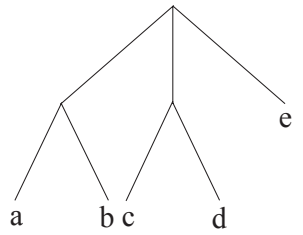

(c)

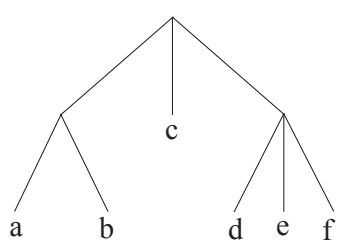

(d)

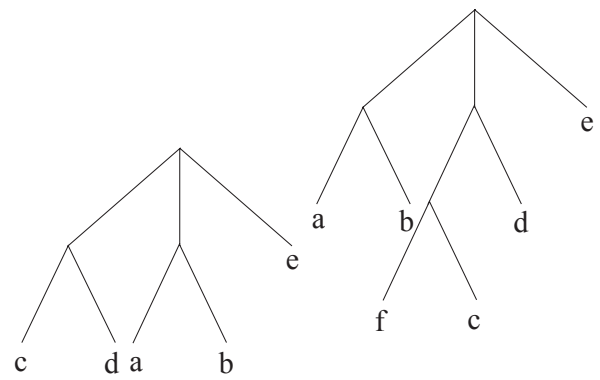

(e) (f)

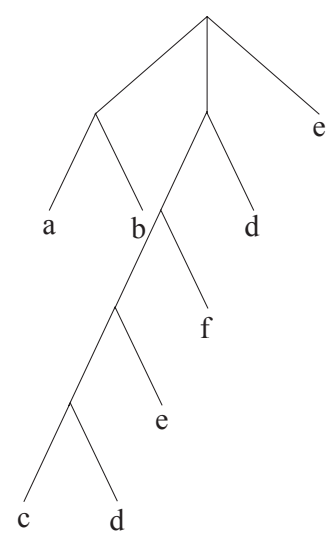

(g)

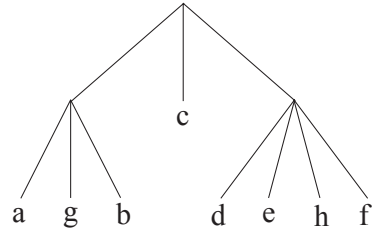

(h)

Figure 24: Examples of Trees for Distance Computation

Table 2: Distance between trees in Figure 24

\begin{tabular}{|l||c|c|c|c|}
\hline Trees & $(a),(e)$ & $(b),(f)$ & $(c),(g)$ & $(d),(h)$ \\
\hline Distance & 2 & 1 & $4.0 \dot{3}$ & 2 \\
\hline
\end{tabular}

The distances between the left and right trees ${ }^{4}$ in each column in Fig. 24 are listed in Table 2, since

$$
\begin{aligned}
& D((a),(e))=\frac{1}{1+0} \cdot 2=2 \\
& D((b),(f))=\frac{1}{1+2} \cdot 3=1 \\
& D((c),(g))=\frac{1}{1+2} \cdot 10+\frac{1}{1+4} \cdot 1+\frac{1}{1+3} \cdot 1+\frac{1}{1+3} \cdot 1=4.0 \dot{3}, \\
& D((d),(h))=\frac{1}{1+0} \cdot 2=2 .
\end{aligned}
$$

In these examples, the cost for the transform of alphabet is unity.

\section{Hierarchy of Temporal Image Sequence}

We apply the scale-space analysis to moving images. Given a moving image or a sequence of images, we can construct the scale-space tree for each image frame. The major advantages of motion analysis using the scale-space tree are as follows.

Nonempirical observation without prior knowledge. The motion of the stationary points strongly depends on the motion of objects in the image. The stationary points are mathematically welldefined feature points of the image, and the structure of the image is wholly described by the

\footnotetext{
${ }^{4}$ These examples are cited from ref. (Nishida, 1994).
} 
differential geometric properties of the scale-space image. Therefore, we can observe the objects from a mathematical point of view without the need of a prior knowledge about the objects and their motion.

Detection of qualitative changes. A sequence of scale-space trees are derived from a sequence of images. Temporal changes in the hierarchical structure of the image are detected as the transition of the scale-space tree with respect to time. We can segment the image sequence into subsequences using the qualitative changes in the image sequence.

Scale selection. A coarse to fine approach is possible. Transitions of the dominant parts of the image changes the hierarchical structure at coarse levels of the scale. That is, a few levels of scale-space trees are sufficient to identify critical variations of the image. Although more stationary points can be found at finer scales, these points are related to background noise and texture spots. They are randomly distributed in image regions with higher scales. Since the randomness is filtered out as the scale increases, the deterministic structure of the image appears at coarse scales.

Tree analysis. Conventional tree analyses are applicable. The magnitude of topological changes in the image can be quantified by the tree edit distance, for example.

We present experimental results of the motion analysis using the scale-space tree for three sequences of images: beating heart, rotating box, and kicking horse.

Beating Heart Figure 25 shows a preliminary analysis of an image sequence of a beating heart. The sequence of stationary curves in scale space is displayed together with the frame images. The annihilation points and stationary points at $\tau=500$ are plotted on the each frame image, where the corresponding scales are expressed by the radii of the circles.

The temporal tree analysis found that from frames 6 to 7 , from frames 7 to 8 , from frames 11 to 12 , from frames 12 to 13 , from frames 31 to 32 , and from frames 32 and 33, gray-values of images are topologically different, and that from frames 1 to 6 , from frames 8 to 11, from frames 13 to 31 , and from frames 33 to 38 , the gray values of images are topologically equivalent. These topological transitions where extracted using the tree metric for trees shown in Fig. 25. The distances among trees which are topologically different are

$$
\begin{array}{ll}
D(T(6), T(7))=1.5, & D(T(7), T(8))=3, \\
D(T(11), T(12))=3, & D(T(12), T(13))=1.5, \\
D(T(31), T(32))=1.5, & D(T(32), T(33))=1.5 .
\end{array}
$$

These distances achieves the grouping of images in a sequence as

Frames 0 to $6, \quad$ Frame 7, Frames 8 to 11, Frame 12, Frames 13 to 31 , Frame 32, Frames 33 to 38 .

The image sequence is segmented into seven subsequences according to the changes of hierarchical structure. We see a drastic change of the structure when the heart shrinks around the 12th frame, in which the trunk curve switches back and forth between two brightest parts of the heart. The temporal scale-space analysis achieves the detection of the topological changes in images of a beating heart. This result indicates that our temporal scale-space analysis based on tree construction in the scale space is feasible for the sequential analysis of low-contrast medical images.

Rotating Box Figure 26(a) shows a sequence of a rotating box (UMass, 2010). The sequence consists of 29 frames, in which a box with rectangular faces rotates from left to right. The image sequence is segmented into four subsequences according to three major transitions of the scale-space tree constructed at coarse scales. The first subsequence continues until the right face of the box disappears. In the second subsequence, the front and top faces are dominant. The third subsequence shows the appearance of the left face. After the switch of the trunk curve, the fourth subsequence starts.

The scale-space trees corresponding to the four subsequences are shown in Fig. 26(b), where we omit some branches that are not related to the topological changes of the tree throughout the sequence. In the first transition, nodes for the annihilation points of local extrema $\mathrm{R}$ corresponding 


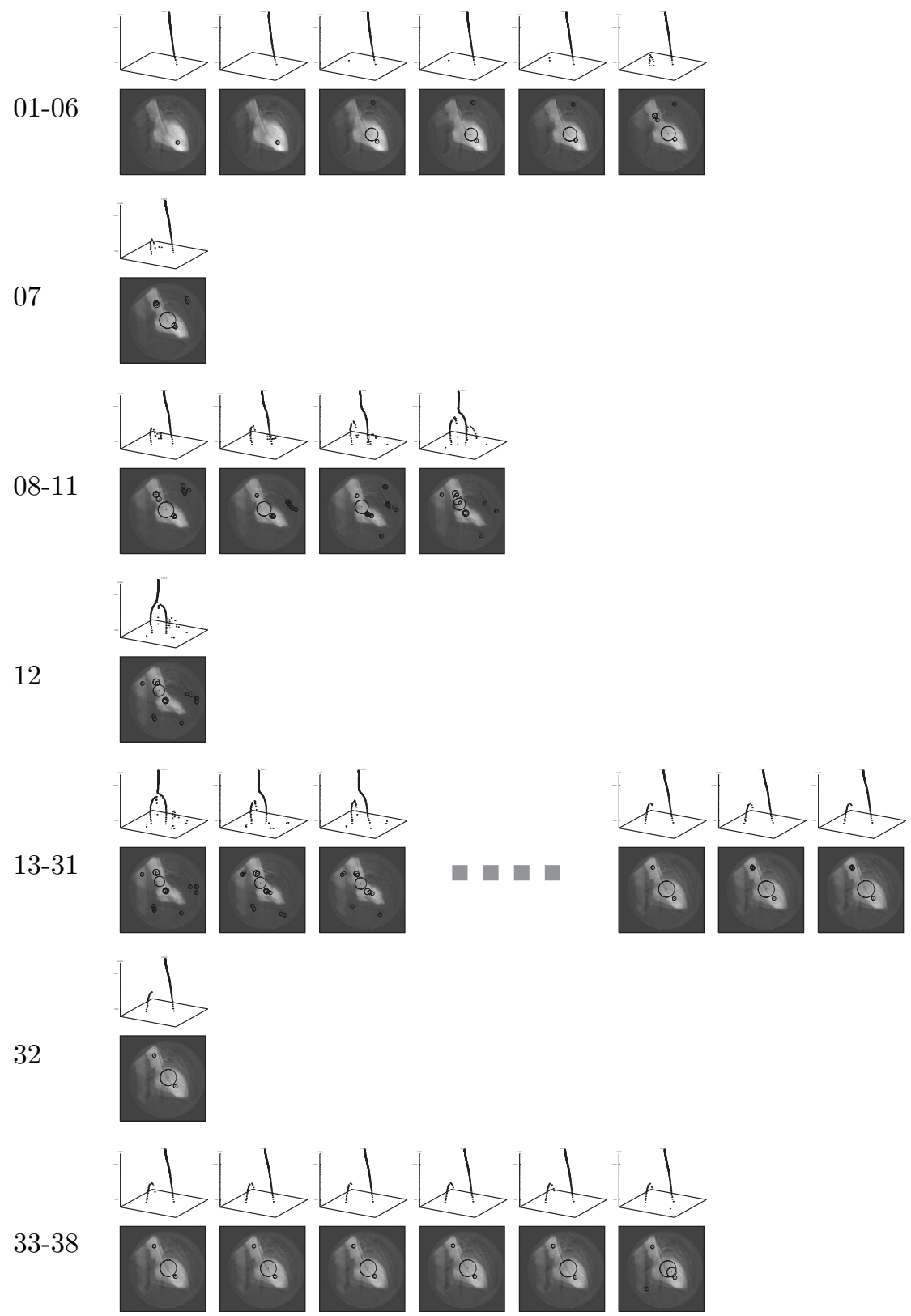

Figure 25: Temporal segmentation of an image sequence of a beating heart with 38 frames. 

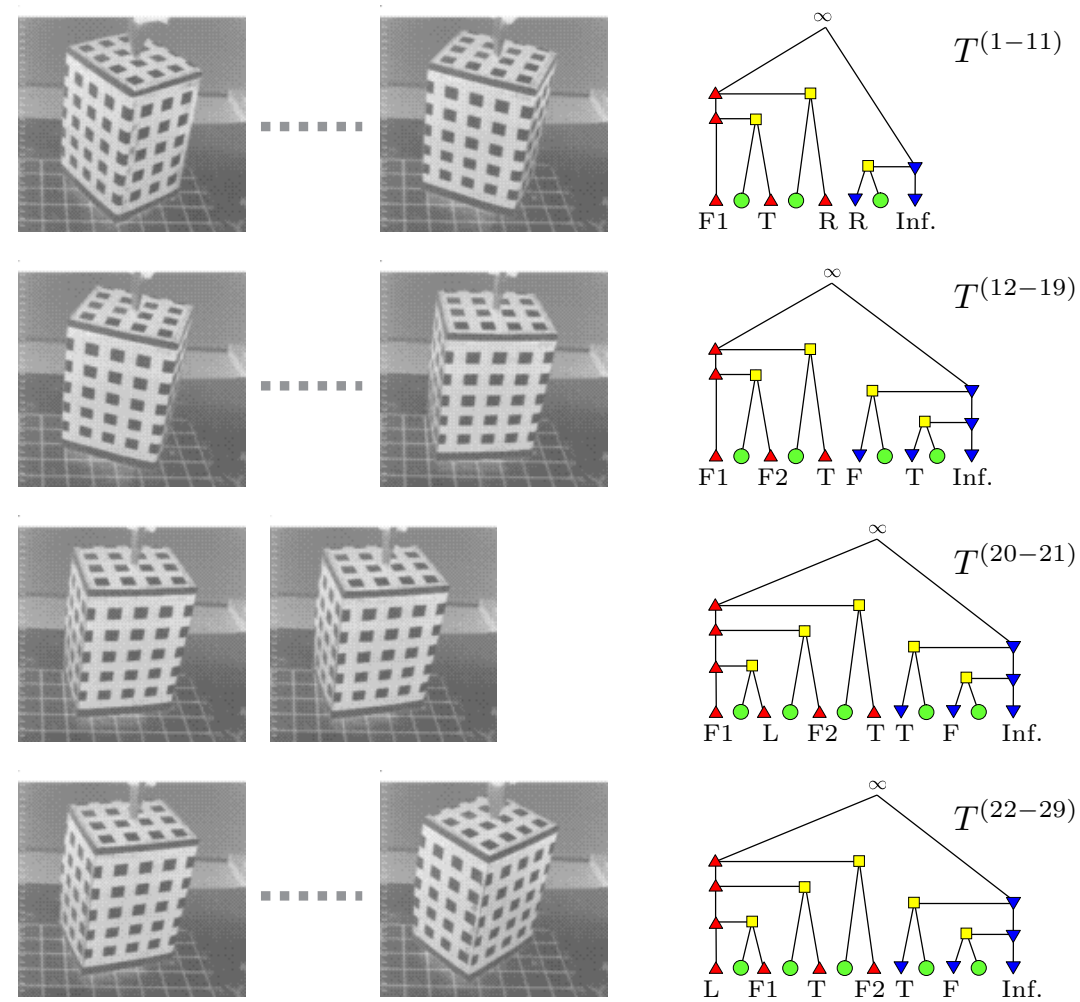

(a)

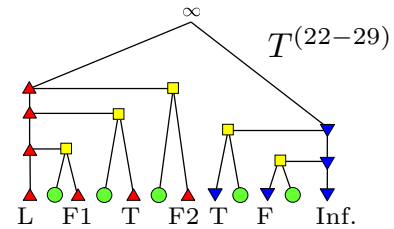

(b)

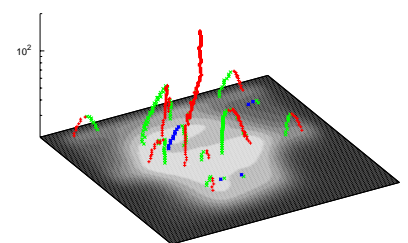

The 21st frame

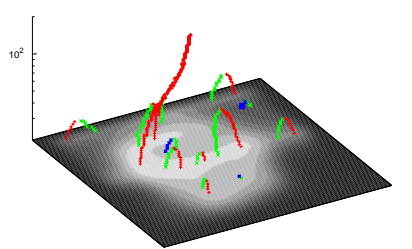

The 22nd frame

(c)

Figure 26: Temporal segmentation of an image sequence of a rotating box. (a) First row: 1st to 11th frames; second row: 12th to 19th frames, third row: 20th and 21st frames; and fourth row: 22nd to 29th frames. (b) Scale-space trees corresponding to the four subsequences in (a). (c) Switch of trunk curve between 21 st and 22 nd frames. The stationary curves $\boldsymbol{x}(\tau \geq 30)$ and the scale-space images $f\left(x_{1}, x_{2}, \tau=30\right)$ are shown for both frames.

to the right face of the box disappear from the tree after the 12 th frame. In the second subsequence, new local minima $\mathrm{F}$ and $\mathrm{T}$, and a local maximum $\mathrm{F} 2$ are enhanced in the from face and top face of the box. After the 20th frame, a node for a local maximum L corresponding to the appearing left face is appended to the tree. We find that the trunk curve switches the connection from the local maximum in the front face to that in the left face between the 21st and 22nd frames. The stationary curves in scale space are shown for these two frames in Fig. 26(c). The brightest part of the image jumps from the front face to the coming left face, as if the attention point moves to the new dominant part of the image. Therefore, this switch of the trunk curve can be interpreted as a jump of the geometric cue of the image from the front face to the coming left face of the box.

Kicking Horse Figure 27 shows the stationary curves in scale space for 16 frames of an image sequence of a horse in a kicking motion in ref. (Muybridge, 1887). The trunk curve starts at the centre of the body of the horse except $t=5,6,15$, at which all hooves are on the ground. That is, the kicking phase is distinguished from that of standing on the ground by the switch of trunk curve without any prior knowledge of the sequence. In the kicking phase, the scale-space hierarchy of stationary points properly expresses the structure of the apparent shape of the horse. In the 9 th 


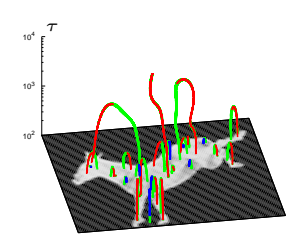

$t=1$

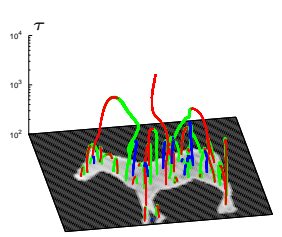

$t=5$

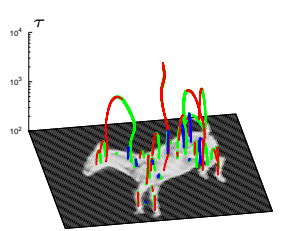

$t=9$

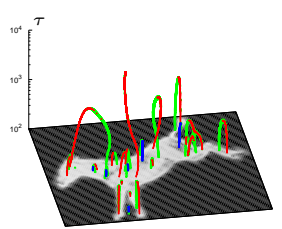

$t=13$

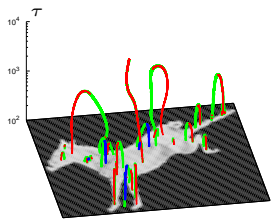

$t=2$

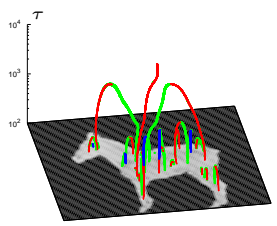

$t=6$

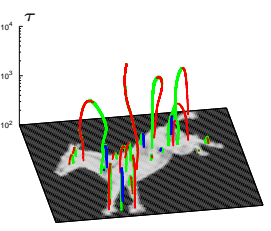

$t=10$

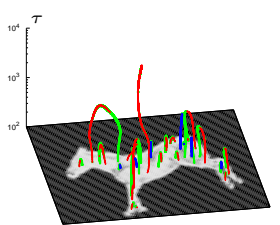

$t=14$

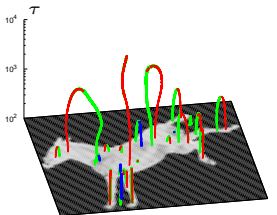

$t=3$

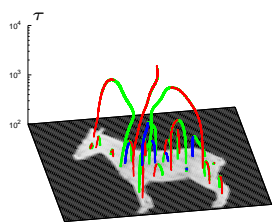

$t=7$

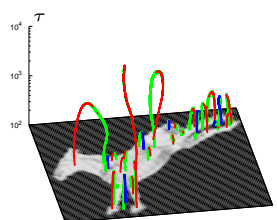

$t=11$

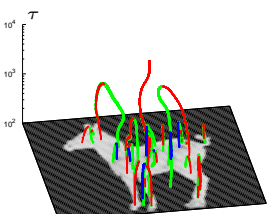

$t=8$

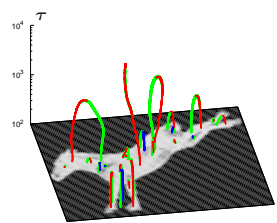

$t=12$

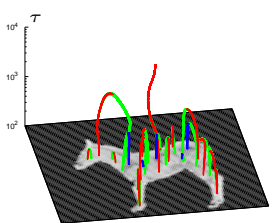

$t=15$

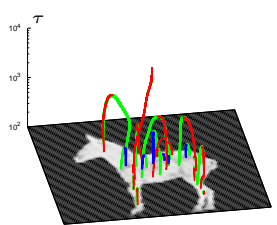

$t=16$

Figure 27: Stationary curves for an image sequence of a kicking horse from Animal Locomotion (Muybridge, 1887)

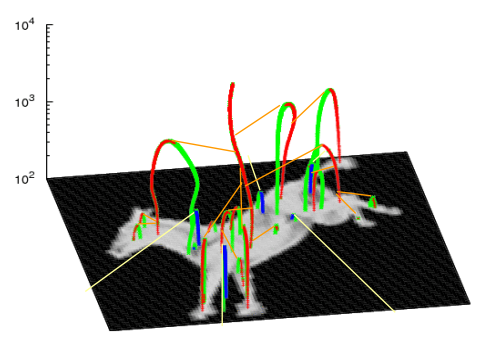

(a)

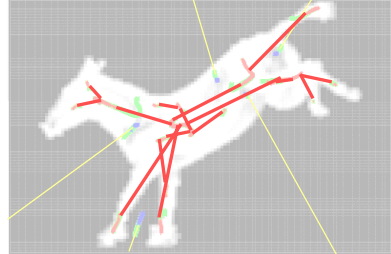

(b)

Figure 28: Structure of image in the 9th frame. (a) Stationary curves for an image sequence of a kicking horse. The straight lines indicate the links between the annihilation points and local extrema. (b) Top view of scale space. 
frame, for example, the topology of stationary points suggests that the hip, head, shoulder, and hind legs are directly connected to the centre of the body. The forelegs and tail are connected to the shoulder and hip, respectively. We exhibit the stationary curves and links of the annihilation points in scale space for the 9th frame in Fig. 28 instead of the scale-space tree. The straight lines in scale space indicate the links between the annihilation points and local extrema identified by the antidirectional figure-flow curves. As shown in Fig. 28(b), The shape of the horse is roughly skeletonised by the local maximum curves and the links of the annihilation points. This skeletal expression can be refined if we trace the exact paths of the antidirectional figure-flow curves.

Neither an accurate measurement nor the identification of objects in the image is required for the present motion analysis. That is, we achieve the motion analysis without the need of any model or a priori information about the target objects and scene. If we limited the target to human motion, the positions and scales of the major parts of the human body could be automatically estimated together with the hierarchical structure. Further analyses, such as manifold learning in the multidimensional space of the estimated parameters, would be possible, and we could deal with topological and geometric information, simultaneously.

\section{Segment Hierarchy}

\subsection{Segmentation in Scale Space}

For a two-argument function $z=f(x, y)$ defined on $\overline{\mathbb{R}}^{2}$, Enomoto and Katayama (Enomoto and Katayama, 1976), Enomoto, Yonezaki, and Watanabe (Enomoto, Yonezaki, and Watanabe, 1982), and Krueger and Phillips (Krueger and Phillips, 1989) defined three types of second-order singularpoint sets as

$$
\nabla f^{\top} \boldsymbol{H} \nabla f=0, \nabla^{\perp} f^{\top} \boldsymbol{H} \nabla f=0, \nabla^{\perp} f^{\top} \boldsymbol{H} \nabla^{\perp} f^{\top}=0,
$$

where $\nabla^{\perp} f^{\top} \nabla f=0$, that is, for $\nabla f=\left(f_{x}, f_{y}\right)^{\top}, \nabla^{\perp} f=\left(-f_{y}, f_{x}\right)^{\top}$. Enomoto and Katayama (Enomoto and Katayama, 1976) called the point sets

$$
\begin{aligned}
E & =\left\{\boldsymbol{x} \mid \nabla f^{\top} \boldsymbol{H} \nabla f=0\right\}, \\
C & =\left\{\boldsymbol{x} \mid \nabla^{\perp} f^{\top} \boldsymbol{H} \nabla f=0\right\}, \\
D & =\left\{\boldsymbol{x} \mid \nabla^{\perp} f^{\top} \boldsymbol{H} \nabla^{\perp} f^{\top}=0\right\},
\end{aligned}
$$

the edge, characteristic, and division lines, respectively.

Setting $\boldsymbol{n}$ and $\boldsymbol{t}$ to be the normal and tangent vectors on the iso-level contour curves of surface $f(x, y)-z=0$, points on $E, C$, and $D$, satisfy the following geometrical properties (Enomoto and Katayama, 1976; Enomoto, Yonezaki, and Watanabe, 1982; Krueger and Phillips, 1989).

- On $E, \nabla f=0$ or the normal curvature on the surface $f(x, y)-z=0$ for the direction of $\nabla f$ is zero. On $E, \frac{d}{d \boldsymbol{n}}|\nabla f|=0$ since

$$
\frac{d^{2}}{d \boldsymbol{n}^{2}}|\nabla f|=\frac{1}{|\nabla f|^{2}} \nabla f^{\top} \boldsymbol{H} \nabla f
$$

- On $C$, an eigenvector of $\boldsymbol{H}$ is $\nabla f$, since $\nabla^{\perp} f^{\top} \nabla f=0$ and $\nabla^{\perp} f^{\top} \boldsymbol{H} \nabla f$ imply that $\boldsymbol{H} \nabla f=$ $\alpha \nabla f$ for $\alpha \in \mathbf{R}$. On $C, \frac{d}{d \boldsymbol{t}}|\nabla f|=0$ since

$$
\frac{d^{2}}{d \boldsymbol{t}^{2}}|\nabla f|=\frac{1}{|\nabla f|^{2}} \nabla^{\perp} f^{\top} \boldsymbol{H} \nabla f .
$$

- On $D \nabla f=0$ or the normal curvature on the surface $f(x, y)-z=0$ for the direction of $f(x, y)=$ const. is zero. On $D, \frac{d}{d \boldsymbol{t}} \frac{f_{x}}{f_{y}}=0$ since

$$
\frac{d^{2}}{d \boldsymbol{t}^{2}} \frac{f_{x}}{f_{y}}=\frac{1}{|\nabla f|^{2}} \nabla^{\perp} f^{\top} \boldsymbol{H} \nabla^{\perp} f .
$$

Krueger and Phillips (Krueger and Phillips, 1989) showed that $C$ describes the local symmetry and $E$ indicates the edge of a segment. We define structure lines in the linear scale space. 


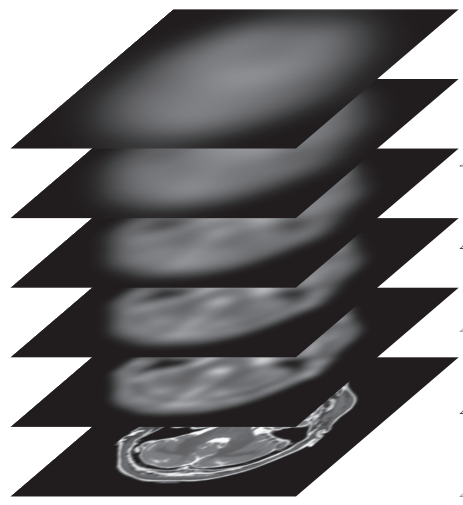

(a) Input images

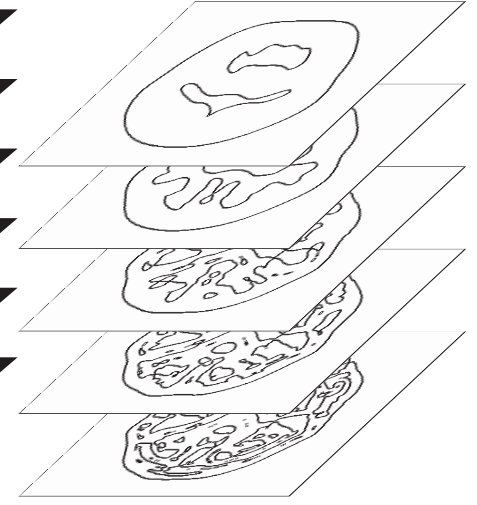

(b) Edge lines

Figure 29: Hierarchy of edge lines in the Gaussian scale space. (a) Multiresolution expression of an image in the Gaussian scale space. (b) Multiresolution expreesion of edge lines in the Gaussian scale space.

Definition 15 Structure lines in the Gaussian scale space $E(\tau), C(\tau)$, and $D(\tau)$ are

$$
\begin{aligned}
E(\tau) & =\left\{\boldsymbol{x} \mid \nabla_{G} f^{\top} \boldsymbol{H}_{G} \nabla_{G} f=0\right\}, \\
C(\tau) & =\left\{\boldsymbol{x} \mid \nabla_{G}^{\perp} f^{\top} \boldsymbol{H}_{G} \nabla_{G} f=0\right\}, \\
D(\tau) & =\left\{\boldsymbol{x} \mid \nabla_{G}^{\perp} f^{\top} \boldsymbol{H}_{G} \nabla_{G}^{\perp} f^{\top}=0\right\} .
\end{aligned}
$$

These definitions correspond to the definition of zero-crossing of Krueger and Phillips (Krueger and Phillips, 1989). Figure 29 shows multiscale expression of $E$-lines in the Gaussian scale space. In this section, we investigate a hierarchical property of segments encircled by $E$-lines.

For the zero-crossing set of a function $f(\boldsymbol{x})$,

$$
\mathbf{A}=\{\boldsymbol{x} \mid f(\boldsymbol{x})=0\},
$$

we define $\phi(\mathbf{A})$ as

$$
\phi(\mathbf{A})=\{\boldsymbol{x} \mid \boldsymbol{x}=\phi(\boldsymbol{y}), f(\boldsymbol{y})=0\}
$$

for an invertible transformation $\phi$. Setting $E[f]$ to be the operation to derive $E$-lines of $f$ and $\phi \circ f(x, y)=f(\phi(x, y))$, Eq. (100) implies the relation .

Lemma 2 For an invertible function $\phi$ and a function $f$ defined on $\overline{\mathbb{R}}^{2}$, the relation

$$
\phi \circ E[f]=E[\phi \circ f]
$$

is satisfied.

\section{Proof 3 Since}

$$
\nabla(\phi \circ f(x, y))=\boldsymbol{J}_{\phi}^{-\top} \nabla f(x, y), \boldsymbol{H}_{\phi \circ f}=\boldsymbol{J} \boldsymbol{H} \boldsymbol{J}^{\top}
$$

for the Jacobian $\boldsymbol{J}$ of $f$, we have the equality

$$
\nabla(\phi \circ f)-\top \boldsymbol{H}_{\phi \circ f} \nabla(\phi \circ f)=\left(\boldsymbol{J}_{\phi}^{-\top} \nabla f\right)^{\top} \boldsymbol{J} \boldsymbol{H} \boldsymbol{J}^{\top}\left(\boldsymbol{J}_{\phi}^{-\top} \nabla f\right)=\nabla f^{\top} \boldsymbol{H} \nabla f .
$$

This relation implies that $\phi(E(f))=E(\phi \circ f)$.

If $\phi$ is a pure rotation, we have the following relation.

Lemma 3 The rotation of $E(f)$ is the E-line of the rotation of $f$.

The same relations are satisfied for $C$-lines and $D$-lines.

Setting $R f(\boldsymbol{x})=f\left(\boldsymbol{R}^{\top} f\right)$, we have the relation $\boldsymbol{R} f(\boldsymbol{x}, \tau)=R\left(G_{\tau} * f\right)=G_{\tau} * R f$. Therefore, we have the following relation. 


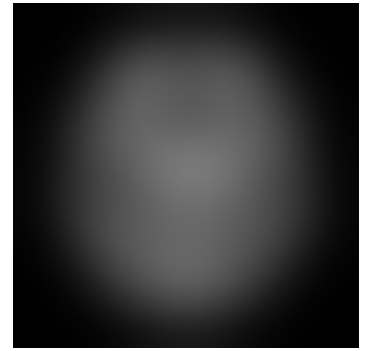

(a) $f(x, 1000)$

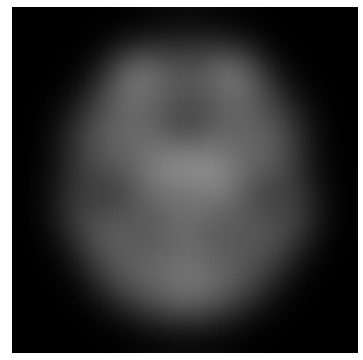

(e) $f(x, 500)$

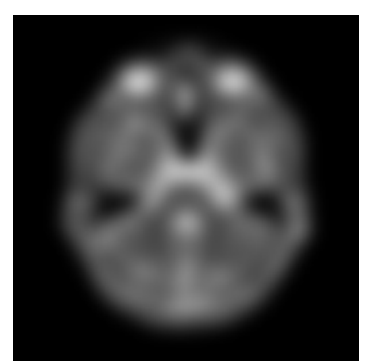

(i) $f(x, 100)$

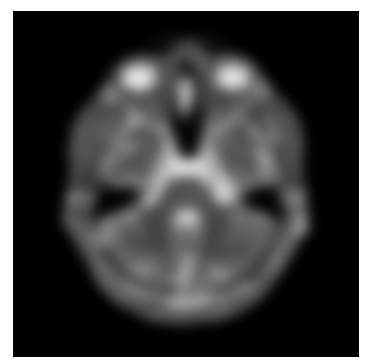

(m) $f(x, 50)$

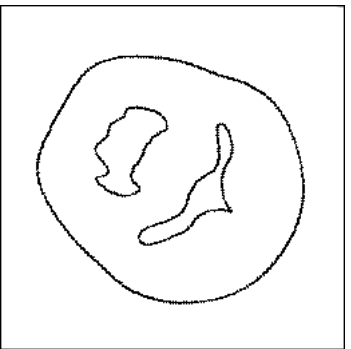

(b) $R(E f(x, 1000))$

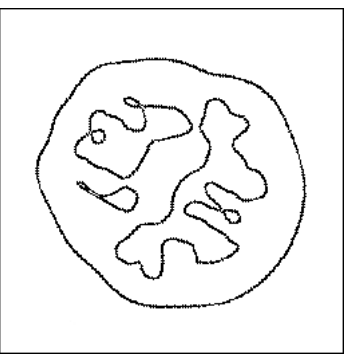

(f) $R(E f(x, 500))$

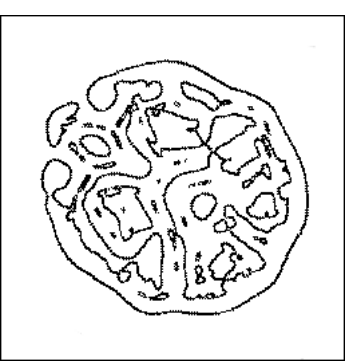

(j) $R(E f(x, 100))$

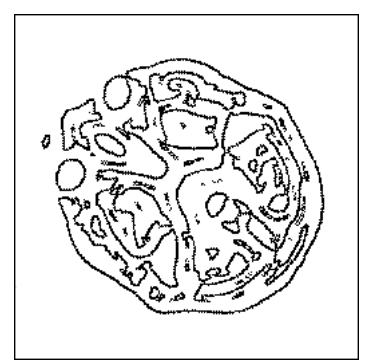

(n) $R(E f(x, 50))$

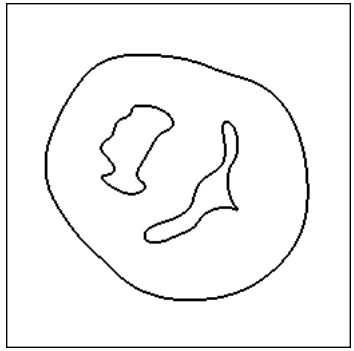

(c) $E(R f(x, 1000))$

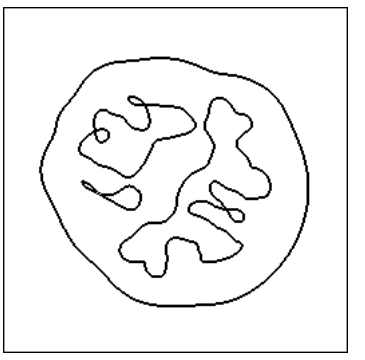

(g) $E(R f(x, 500))$

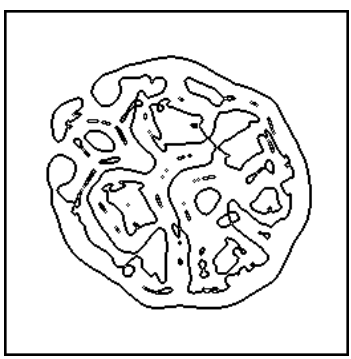

(k) $E(R f(x, 100))$

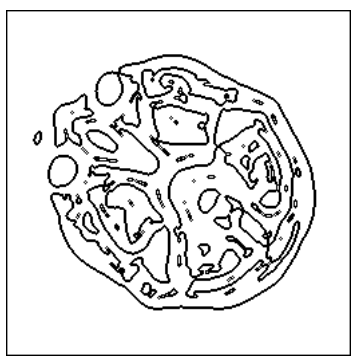

(o) $E(R f(x, 50))$

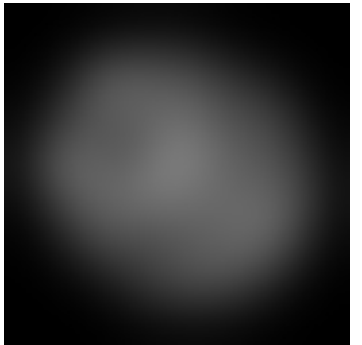

(d) $R f(x, 1000)$

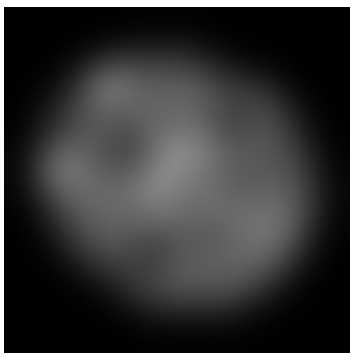

(h) $R f(x, 500)$

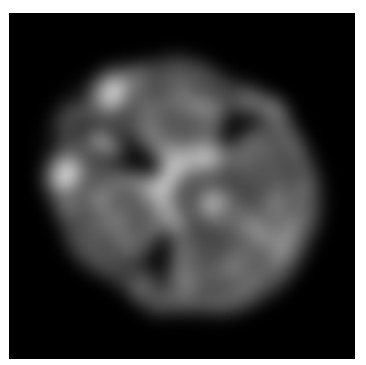

(l) $R f(x, 100)$

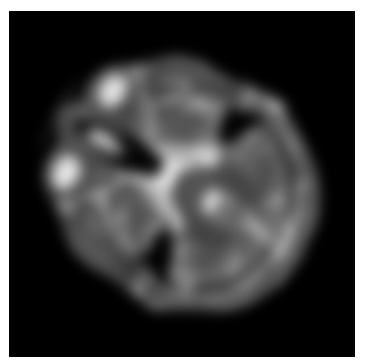

(p) $R f(x, 50)$

Figure 30: Edge lines of rotated images in the Gaussian scale space. From the left column to right column, multiresolution image representation in the Gaussian scale space, edge lines of images in the first column, edge lines of multiscale images after rotation shown in the fourth column.

Lemma 4 Assuming that $E(\tau ; R)$ is the edge lines of $R f$, the relation

$$
R E(\tau)=E(\tau ; R)
$$

is satisfied.

Figure 30 shows the relation of Lemma 3 in the Gaussian scale space. The first and second columns show a series of multiscale images in the Gaussian scale space and the rotated $E$-lines of 
images in the first column, respectively. The fourth and third columns show a series of rotated images in the linear scale space and $E$-lines extracted images in the fourth column. The series of $E$-lines in the second and third columns coincide within numerical errors mainly caused by the rotation of images in the discrete space.

If

$$
f_{\pi}(x)=f(\boldsymbol{x}) \delta\left(\boldsymbol{\omega}^{\top} \boldsymbol{x}-a\right)
$$

$f_{\pi}$ is the restriction of $f$ to the plane

$$
\Pi=\left\{\boldsymbol{x}\left|\boldsymbol{x}^{\top} \boldsymbol{\omega}=a,\right| \boldsymbol{\omega} \mid=1, a \geq 0\right\} .
$$

Setting $\nabla_{\Pi} f$ and $\boldsymbol{H}_{\Pi}$ to be the gradient and the Hessian of $f$ on plane $\Pi$, we have the following lemma.

Lemma 5 Iff either $\frac{\partial f}{\partial \boldsymbol{\omega}}=0$ or $f(\boldsymbol{x})=0$ for $\boldsymbol{x} \notin \Pi$, we have the relation

$$
\nabla_{\pi} f_{G}^{\top}\left(\boldsymbol{H}_{\pi}\right)_{G} \nabla_{\Pi} f_{G}=\nabla f_{G} \boldsymbol{H}_{G} \nabla f_{G}
$$

Proof 4 Without loss of generality, we can set $\boldsymbol{\omega}=(0,0, \cdots, 1)^{\top}$ and $a=0$. Therefore, if $\frac{\partial f}{\partial \boldsymbol{\omega}}=0$ or $f(\boldsymbol{x})=0$ for $\boldsymbol{x} \notin \Pi, \frac{\partial f}{\partial x_{N}}=0$ and $\frac{\partial^{2} f}{\partial x_{N} \partial x_{i}}=0$ for $i=1,2, \cdots, N$. Conversely, if Eq. (10 r) is satisfied, $\frac{\partial f}{\partial x_{N}}=0$ and $\frac{\partial^{2} f}{\partial x_{N} \partial x_{i}}=0$. These relations support the statement of the lemma.

Lemma 5 implies that for the extraction of the exact segment of a slice of $3 \mathrm{a} \mathrm{D}$ object, we are required to compute first the edge surface, which is the three-dimensional version of edge lines, and second intersection of the slice plane and edge surface. Furthermore, the edge lines of a slice provide an approximation of the segment boundary on a slice.

\subsection{Segmentation Hierarchy}

For a fixed $\tau, E(\tau)$ is the edge detected by the Canny edge-detection operator (Canny, 1986; Krueger and Phillips, 1989; Najman and Schmitt, 1994). The top-points in the Gaussian scale space satisfy the conditions

$$
\nabla_{G} f=0, \operatorname{det} \boldsymbol{H}_{G}=0 .
$$

Using the geometrical properties of stationary points on stationary curves, we select the set $\left\{\tau_{i}\right\}_{i=1}^{n}$.

Definition 16 We select scales $\left\{\tau_{i}\right\}_{i=1}^{n}$ for the Canny edge detection from among scales at stationary points on stationary curves.

In the neighborhood of a saddle point of $z=f(x, y)$, the terrain surface is approximated by a hyperbola. Assuming a singular point exists at the origin, we have the approximation (Bruce and Giblin, 1992; Rorteous, 2001)

$$
z=a x^{2}-b y^{2}
$$

as shown in Fig. 33. Since

$$
\nabla f^{\top} \boldsymbol{H} \nabla f=8\left(a^{3} x-b^{3} y\right)=0,
$$

in the neighborhood of the origin, the $C$-line is a pair of lines crossing at the origin. The geometrical property leads to the following lemma.

Lemma 6 The $C$ lines crosses at the saddle points in the linear scale space.

Figure 32 shows the relation of $E$ - and $C$ - lines.

We analyse topological property of the $E$-lines when a saddle point merges to a local maximal or minimal point. In the neighborhood of a catastrophic point, the function $f\left(x_{1}, x_{2}, \tau\right)$ is expressed as

$$
f(\boldsymbol{x}, \tau)=x_{1}^{3}+6 x_{1} \tau-\left(x_{2}^{2}+2 \tau\right)
$$

Therefore, the $E$-line is

$$
x_{2}= \pm \sqrt{3^{3} / 2^{2} x_{1}^{5}+3^{3} x_{1}^{3} \tau+3^{3} x_{1} \tau}
$$

Assuming that for $\tau=0$ a pair of a saddle point and an extremal point merged, in Figure 34, lines for $\tau<0$ and $\tau>0$ express lines before and after the merging process, respectively. The curve 


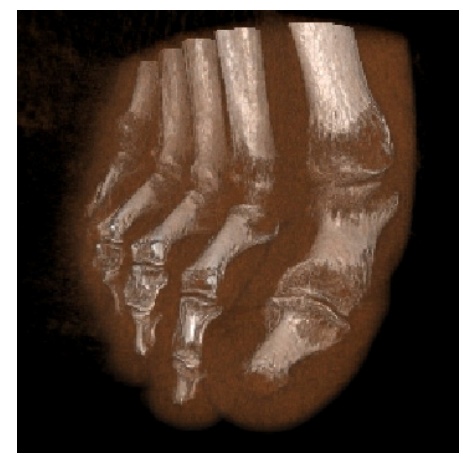

(a) $f(\boldsymbol{x})$

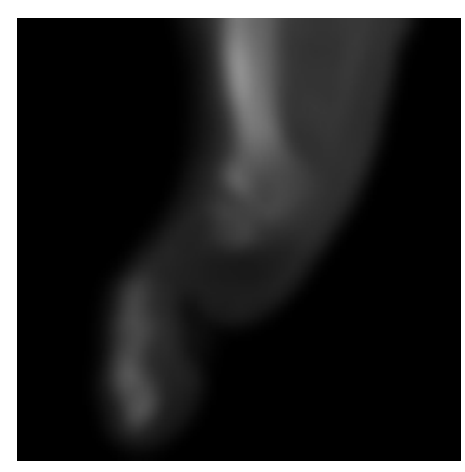

(c) Slice of $f(\boldsymbol{x}, \tau)$

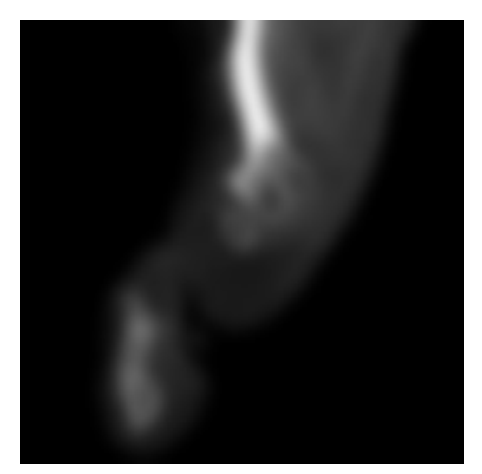

(e) $f_{\pi}(\boldsymbol{x}, \tau)$

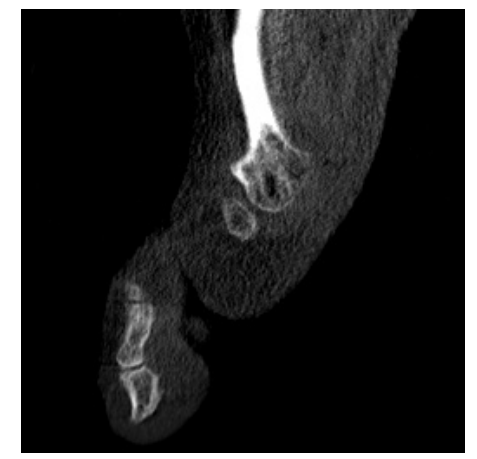

(b) $f_{\pi}(\boldsymbol{x})$

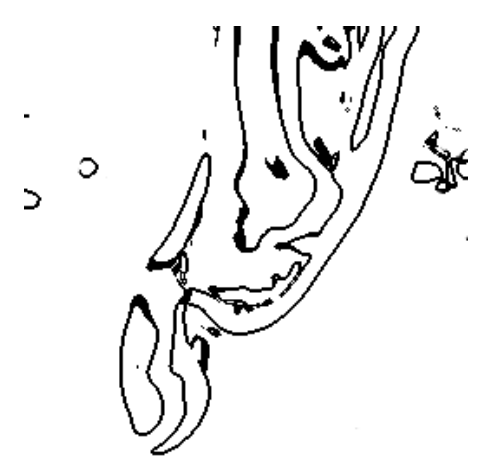

(d) $E_{\tau \pi}$

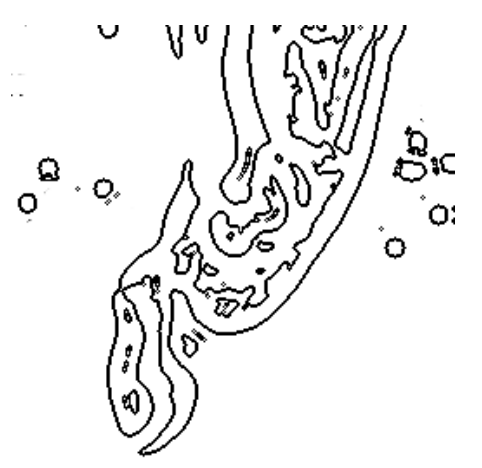

(f) $E_{\pi \tau}$

Figure 31: $E$-lines on a slice. 


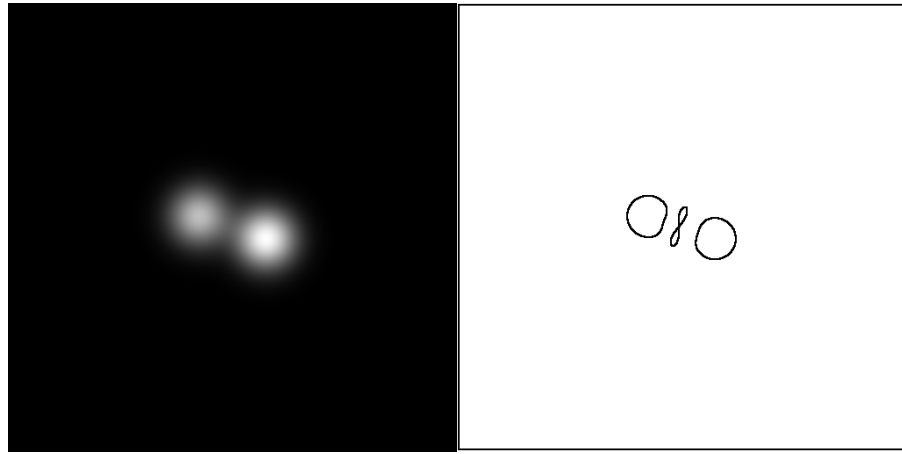

(a) Input image

(b) Edge lines

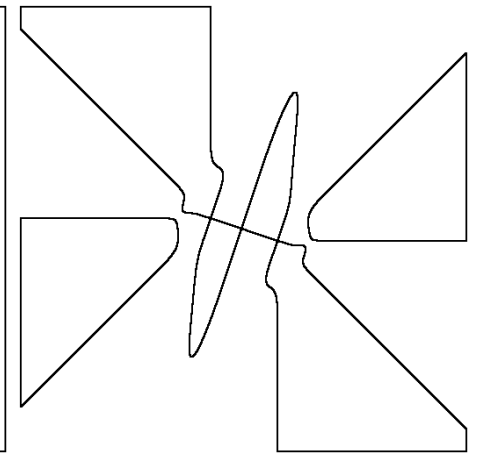

(c) Characteristic lines

Figure 32: Examples of structure lines: (a) input image, (b) edge lines, (c) characteristic lines.

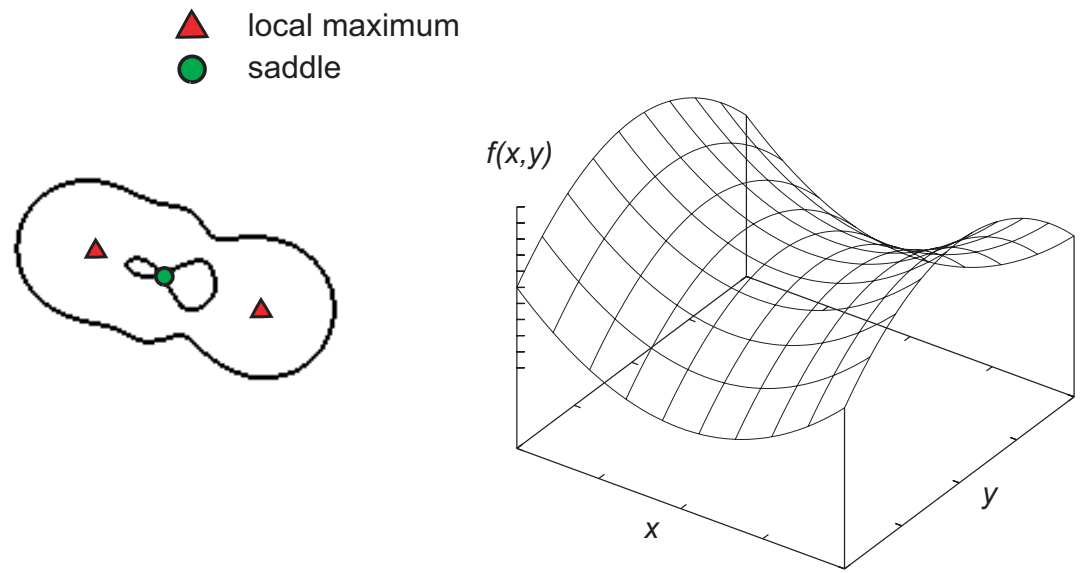

(a)

(b)

Figure 33: Edge lines at a saddle point. (a) Stationary points in Fig. 35(c) at $\tau=220$. (b) Surface plot of $f=a x^{2}-b y^{2}$.

configuration of Fig. 34, which is computed for a geometric image, coincides with that in Fig. 33 (a). This topological property implies assertion 1 .

Let $\sharp(\tau)$ be the number of extrema for the scale $\tau$. Setting $\tau^{*}$ to be a scale which derives a top point, the difference between $\sharp\left(\tau^{*}+\varepsilon\right)$ and $\sharp\left(\tau^{*}-\varepsilon\right)$ is at least one for a small positive constant $\varepsilon$. $E(\tau)$ crosses at saddle points and a simple closed portion of $E(\tau)$ encircles at least one extremal point (Enomoto, Yonezaki, and Watanabe, 1982). These two geometric properties in the linear scale space and topology of $E(\tau)$ lead to the next assertion.

Assertion 1 The difference between the number of simple closed curves for scales $\tau^{*}+\varepsilon$ and $\tau^{*}-\varepsilon$ is at least one.

Figure 35 shows the topological and hierarchical relations of segments extracted from a simple image. Fig. 35(h) shows the merging of a pair of closed structure lines at a top-point.

Assertion 2 A closed curve encircling an extremal has a common region with a stable view field.

From these geometrical properties, we obtain the following proposition.

Proposition 3 If a pair of branches of stationary curves is merged at a top point, a pair of simple closed curves in $E(\tau)$, which share a saddle point is merged into a simple closed curve. 


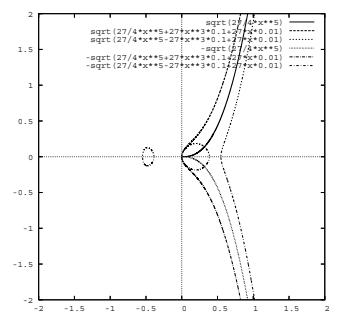

(a) all

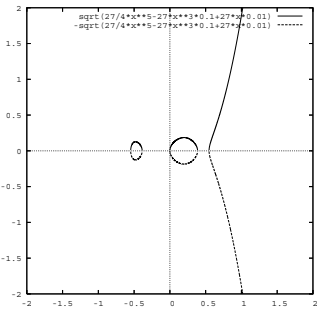

(b) $\tau<0$

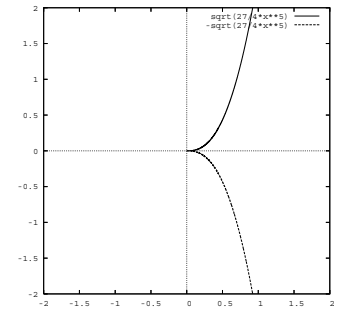

(c) $\tau=0$

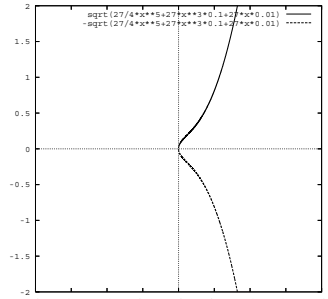

(d) $\tau>0$

Figure 34: Edge lines at the catastrophic point

This property implies the next rule to select scales for the detection of $E(\tau)$ as the segment boundary.

\section{Strategy 1: Parameter Selection for Edge Detection}

1. Compute scales $\left\{\tau *_{i}\right\}_{i=0}^{k}$, which define top points, such that $\tau_{i}^{*} \leq \tau_{i+1}^{*}$ and $\tau_{0}=0$.

2. Select scales in the interval $\left(\tau_{i}^{*}, \tau_{i+1}^{*}\right)$.

We define the hierarchical order of the segments extracted as the zero crossing of $\nabla_{G} f^{\top} \boldsymbol{H}_{G} \nabla_{G}$ for $\left(\tau_{i}^{*}, \tau_{i+1}^{*}\right)$.

\section{Region Tree}

1. For $\tau=\infty$, set the region encircled with a closed loop to be the root of the tree.

2. While decreasing $\tau$, operate the followings;

(2-i) If a new region encircled with a closed loop appears, set this region as the subroot of the node corresponding to the loop encircling the new loop and connect this subroot to the node using an inclusive relation edge.

(2-ii) If a new region incident to a loop appears, set this region as the subroot of the node corresponding to the loop incident to this loop and connect this subroot using an incident relation edge.

Therefore, for a pair of scales separated by a top-point, the topology of $E$-line varies. This property implies the validity of strategy 1 of the selection of scales for the edge detection as zero-closing of $\nabla_{G} f^{\top} \boldsymbol{H}_{G} \nabla_{G} f$. Furthermore, proposition 3 and strategy 1 show that, with careful selection of the Gaussian-kernel variance, the Canny edge-detection algorithm eliminates small segments, which are caused by noise, to avoid over-segmentation. On the other hand, over-smoothing due to a large variance of the Gaussian kernel eliminates some segments and causes under-segmentation. The configuration of top-points, which are second-order singularities in the Gaussian scale space, clarifies the topological change of the segments and boundary curves detected using the Canny edge detection algorithm. 


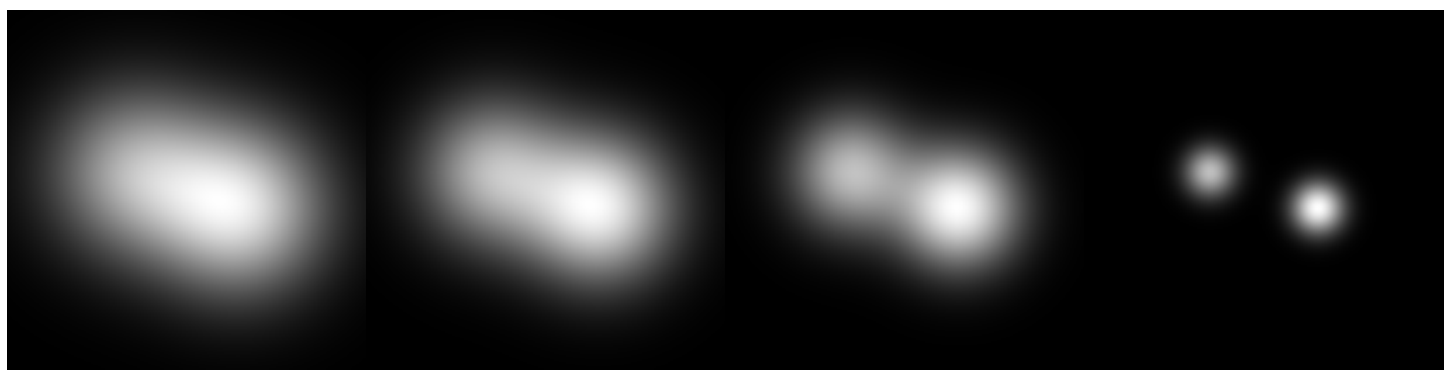

(a) Diffused image; $\tau=$ (b) Diffused image; $\tau=$ (c) Diffused image; $\tau=$ 500
360
220

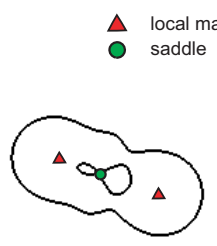

(g) Creation of a region; $\tau=220$ (d) Input image

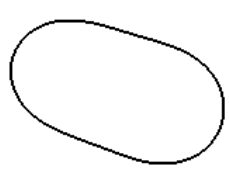

(e) Simple closed curve; $\tau=500$ (f) Creation of a region; $\tau=360$

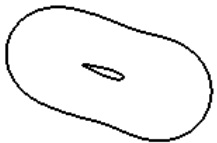

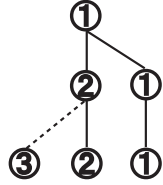

(h) Region tree

Figure 35: Creation of regions with decreasing scale. Top row shows the diffused images of (d), and bottom row shows the edge lines. (e) A simple closed curve at a large scale, (f) creation of region inside existing region, and $(\mathrm{g})$ creation of region which has a contact point. $(\mathrm{h})$ the extracted tree.

Table 3: The number of saddle points for scales.

\begin{tabular}{l|ccccc}
\hline scale & $730>\tau \geq 400$ & $>\tau \geq 380$ & $>\tau \geq 360$ & $>\tau \geq 230$ & $>\tau \geq 220$ \\
\hline $\begin{array}{l}\text { Number of } \\
\text { points }\end{array}$ & 4 & 5 & 6 & 7 & 8
\end{tabular}

\subsection{Experimental Examples}

Figure 36 shows edges and segments for the selected scales. The scales in Fig. 36 are selected based on the numbers of saddle points listed in Table 1 . These numbers define $\tau^{*}$. Figure 37 shows the tree extracted from the singular points in the linear scale space and the tree extracted from segments. The tree constructed from the segments may define a strategy for the unification of small segments into one large segments for the control of oversegmentation. Figure 38 shows the topological relations of regions. These results show that the zero point and zero crossing in the Gaussian scale space yield a hierarchical relation of image segments.

Generally, the set of zero-crossing points $\{\boldsymbol{x} \mid D(\boldsymbol{x})=0\}$ is expressed as a common set of two sets

$$
S_{+}=\{\boldsymbol{x} \mid D(\boldsymbol{x}) \geq 0\}, \quad S_{-}=\{\boldsymbol{x} \mid D(\boldsymbol{x}) \leq 0\} .
$$

In this study, we call $S_{+}$and $S_{-}$the set of positive segments and the set of negative segments, respectively. We show an example of scale evolution of $S_{+}(\tau)$ and $S_{-}(\tau)$ in Fig. 39 .

One of the practical methods of extracting the image segments is Canny's edge detection (Canny, 1986). For each predetermined deviation $\sigma_{i}(i=1, \ldots n)$, edges of the image $f(\boldsymbol{x})$ are detected by the following procedure.

Image Smoothing The image $f(\boldsymbol{x})$ is smoothed using a Gaussian filter with the deviation $\sigma_{i}$ to yield $f_{i}(\boldsymbol{x})=G\left(\boldsymbol{x}, \sigma_{i}\right) * f(\boldsymbol{x})$.

Differentiation Compute the gradient $\nabla f_{i}(\boldsymbol{x}){ }_{44}$ 


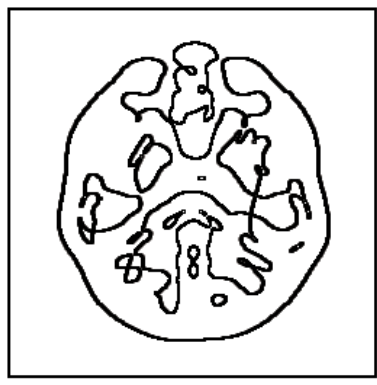

(a) Edge; $\tau=220$

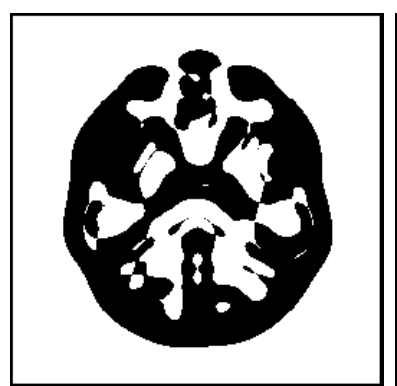

(e) Segment; $\tau=220$

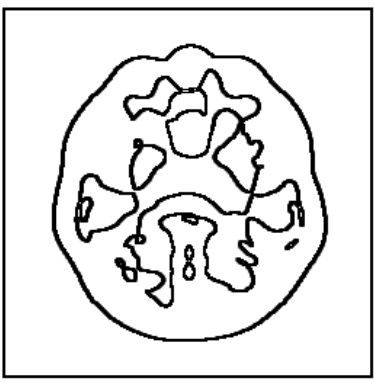

(b) Edge; $\tau=290$

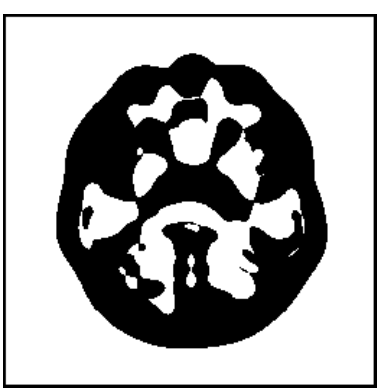

(f) Segment; $\tau=290$

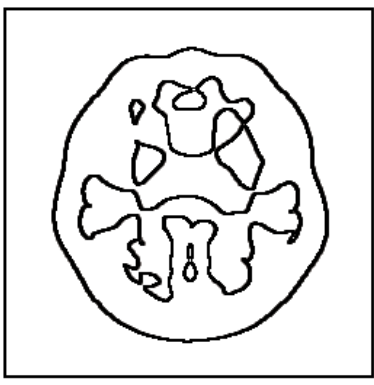

(c) Edge; $\tau=370$

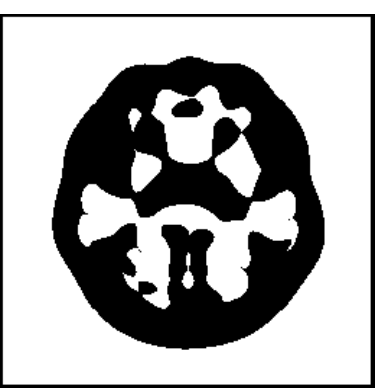

(g) Segment; $\tau=370$

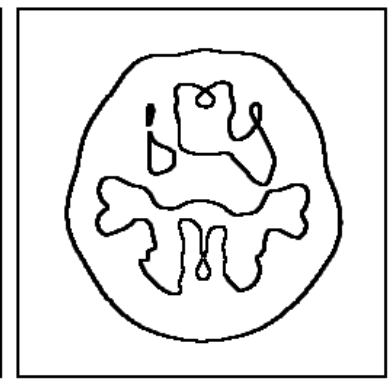

(d) Edge; $\tau=480$

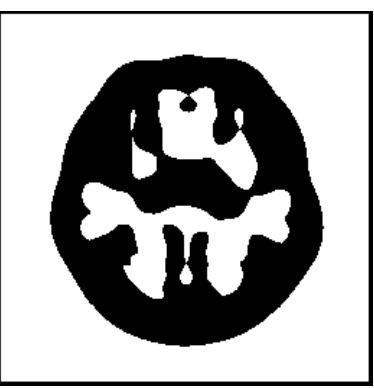

(h) Segment; $\tau=480$

Figure 36: Edges and segments in the Gaussian scale space. From left to right, figures show edges and segments for selected scales.

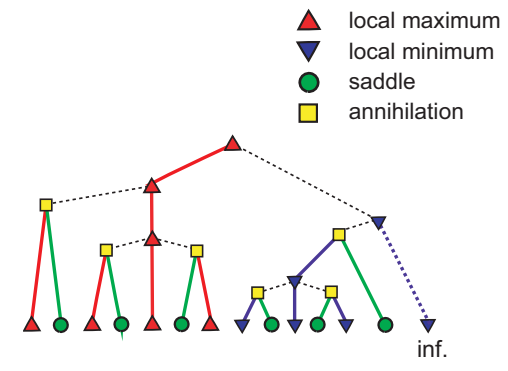

(a) Scale Space tree

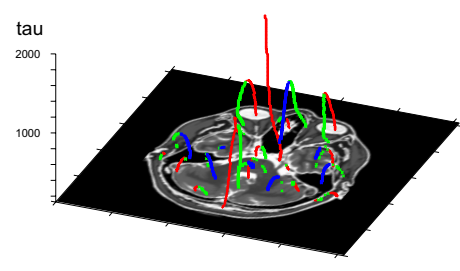

(b) Stationary curve

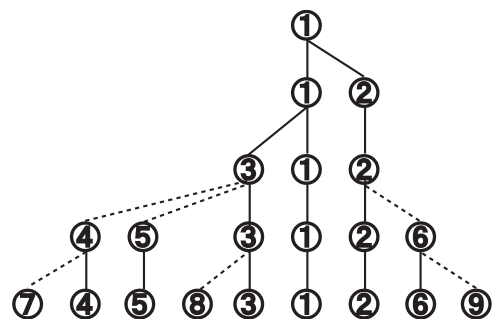

(c) Segment tree

Figure 37: Singular-point tree and segment tree. The tree in (a) is extracted based on the curves in (b). The tree in (c) is extracted from the hierarchy of segments of Fig. 36.

Edge Decision Assume the normal direction $\boldsymbol{n}$ to the edge to be the direction of the gradient, that is, $\boldsymbol{n}=\nabla f_{i}(\boldsymbol{x})$. Edge points are the points of inflection of $f_{i}(\boldsymbol{x})$ in the direction of $\boldsymbol{n}$.

$$
E_{i}=\left\{\boldsymbol{x} \mid \frac{\partial^{2}}{\partial n^{2}} f_{i}(\boldsymbol{x})=0\right\}
$$

In practice, the smoothing and differentiation can be combined into the convolution of Gaussian derivatives. The detection of zero crossing in Eq. (114) is implemented as hysteresis thresholding using a pair of thresholds. The ratio of two thresholds is regulated based on prediction of signal-tonoise ratio of the image.

Canny observed edges with small deviations $\sigma_{i}$. From the viewpoint of the scale-space theory, the Canny edge $E_{i}$ can be regarded as the scale-space version of an edge manifold. Since the directional 


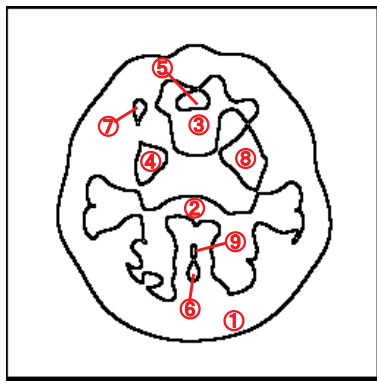

(a) Segment; $\tau=360$

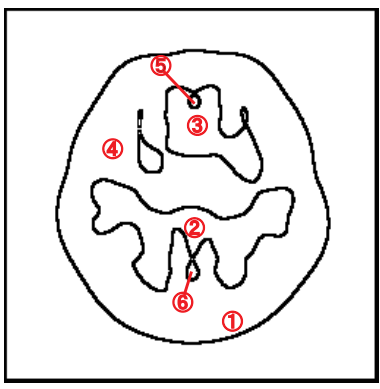

(b) Segment; $\tau=560$

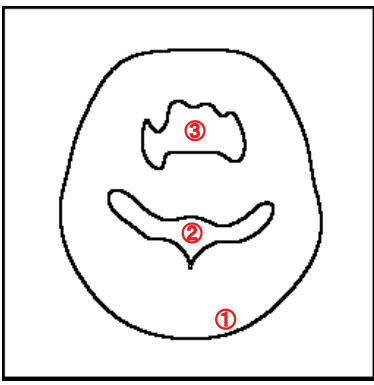

(c) Segment; $\tau=960$

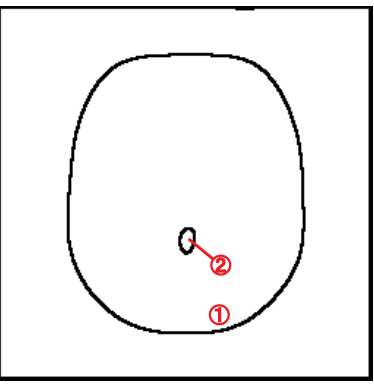

(d) Segment; $\tau=1570$

Figure 38: Edges and segments in the Gaussian scale space. If a closed curve for a small scale encircles a collection of closed curves in a large scale, this relation defines a hierarchy of segments across the scale.

derivative in the direction of $\boldsymbol{n}$ is calculated as $\partial f_{i} / \partial n=\boldsymbol{n}^{\top} \nabla f_{i}$, Eq. (114) can be redescribed as

$$
\frac{\partial^{2} f_{i}}{\partial n^{2}}=\boldsymbol{n}^{\top} \nabla\left(\boldsymbol{n}^{\top} \nabla f_{i}\right)=\boldsymbol{n}^{\top}\left(\nabla \nabla^{\top} f_{i}\right) \boldsymbol{n}
$$

Here, $\nabla f_{i}$ and $\nabla \nabla^{\top} f_{i}$ are the spatial gradient and the Hessian matrix of the Gaussian-smoothed image, that is, the scale-space image.

From these mathematical backgrounds, we have the next property of the edge curves defined by Canny (Canny, 1986).

Assertion 3 The edge of a segment detected by Canny operation (Canny, 1986) is E $(\tau)$, which is the edge-line in the Gaussian scale space for an appropriate scale parameter.

The gradient-based boundary detection detects the gradient set $\left|\nabla T_{\tau} f\right| \geq T$ for a threshold $T$ as the candidate of the boundary point, where $T_{\tau}$ is a scale-space operation. For comparison, Figs 40 and 41 show the segment boundaries in linear and non-linear scale space. After detection the gradient set, the segment boundary is detected by a boundary-following algorithm. The next Canny operation detect the boundary from the gradient set in the Gaussian scale space, since the gradient map of an original image approximates $E(\tau)$.

\section{Canny Edge Detection}

1. Define the parameters $\tau^{*}, T_{1}$ and $T_{2}$ such that $T_{1} \geq T_{2}$.

2. Compute $h=G *_{2} f$.

3. Mark $\theta(x, y)=\tan ^{-1} \frac{h_{x}}{h_{y}}=\tan ^{-1} \frac{G_{x} *_{2} f}{G_{y} *_{2} f}$ on points as edge direction.

4. For $|\nabla h|$, select a point $|\nabla h| \geq T_{1}$ as the starting point of edge tracking.

5. Track peaks using $\theta(x, y)$ defined by $|\nabla h|$ while $|\nabla h| \geq T_{2}$.

A characteristic line is a collection of local symmetry axes (Enomoto, Yonezaki, and Watanabe, 1982). Figure 43 shows the hierarchical property of the characteristic lines. Since the characteristic line passes through the singular point $\operatorname{det} \boldsymbol{H}=0$, it is possible to detect the top-point stably using characteristic lines and edge lines in the Gaussian scale space.

\subsection{Valid Segments}

\subsubsection{Deterministic Structure and Critical Scale}

We expect that the segmentation of scale-space image $f(\boldsymbol{x}, \tau)$ with a small scale $\tau$ well approximates the true image segments. However, if noise spoils the fine structure of the image, or if the image itself has random features such as texture, the estimated image segments are stochastic at small scales. Consequently, $S_{ \pm}(\tau)$ with a small scale is not a feasible estimation. On the other hand, the 

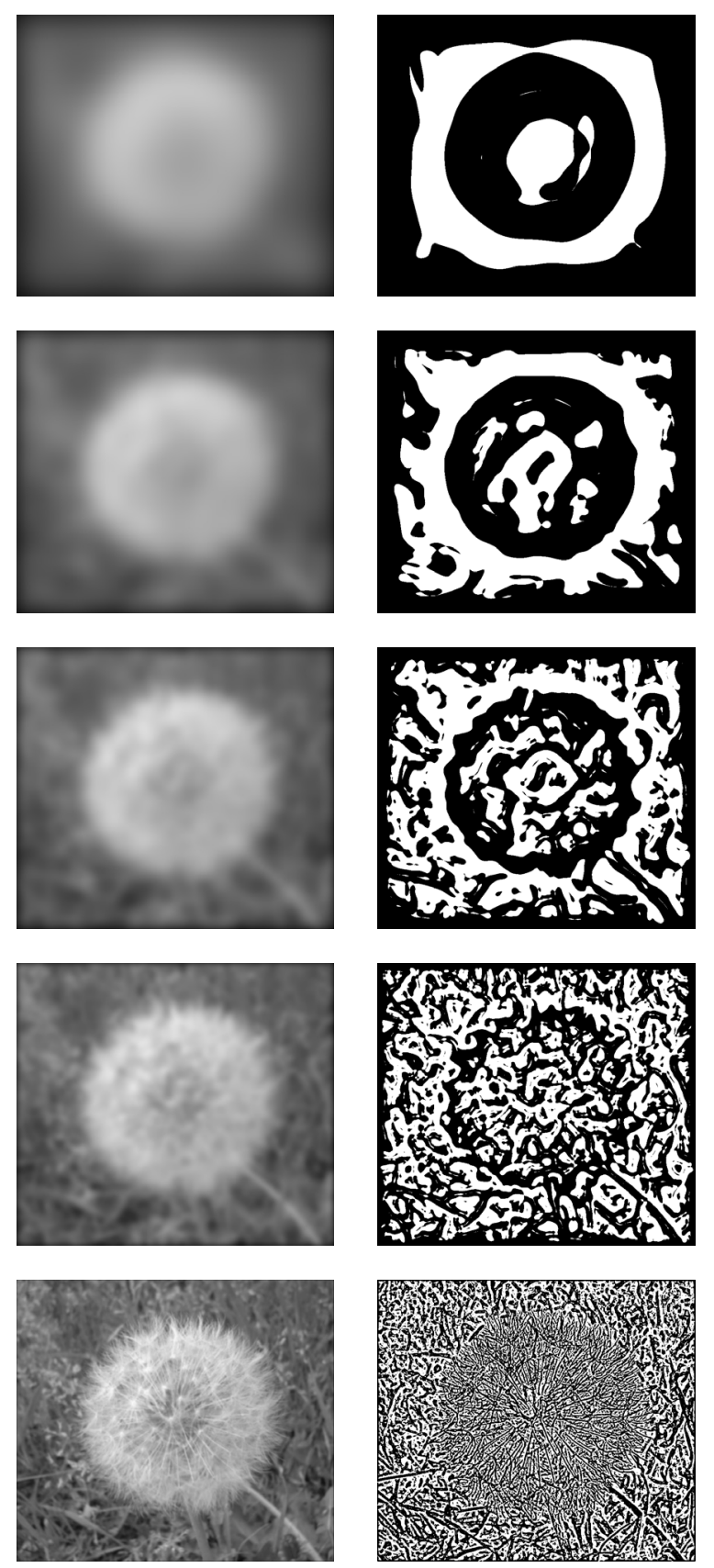

Figure 39: Left: Scale-space image. Right: Segments detected by the Canny edge detector. Black and white regions indicate the negative and positive segments. The scale increases from bottom row to top row.

Canny edge detection with a large scale is poor in terms of detection ability and localisation since the edges of small segments are smoothed out. Consequently, the shapes of boundaries of large segments are simplified. Therefore, we need a criterion to select the scale.

An important fact is that the random features of the image are filtered out as the scale increases, and deterministic features emerge. In other words, the deterministic structure of the image is increasingly established from coarse to fine scales. The structure across scale, so-called DSSS (Witkin, 1983; Koenderink, 1984; Kuijper, Florack, and Viergever, 2003), implies hierarchical relationships among the image features. The scale-space hierarchy of image segments, which we defined in the previous section, provides us with the coarse to fine approach to scale selection for segmentation. There presumably exists a critical lower bound of the scale, above which the structure is deterministic and the segments are valid, and under which the structure is stochastic and the segments are 


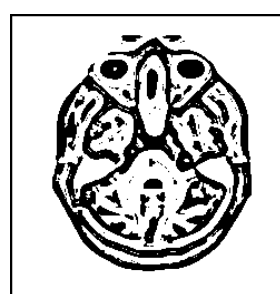

(a) $\tau=010$

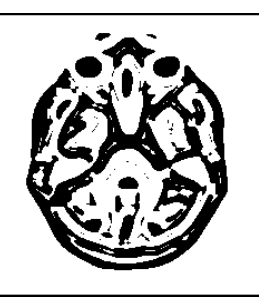

(b) $\tau=020$

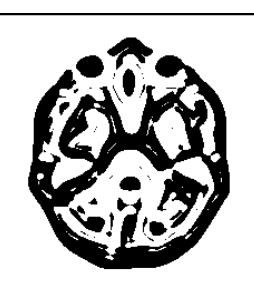

(c) $\tau=030$

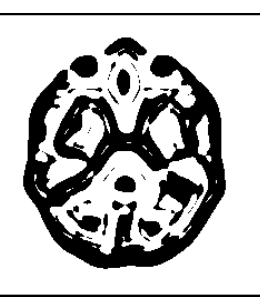

(d) $\tau=040$

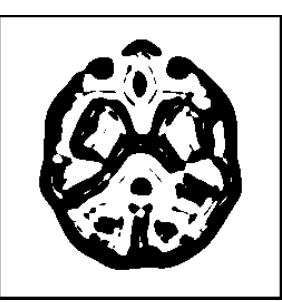

(e) $\tau=050$

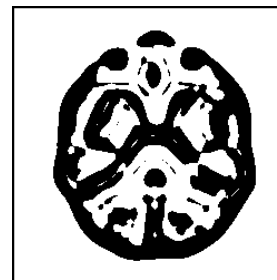

(f) $\tau=060$

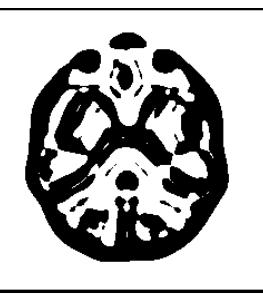

(g) $\tau=070$

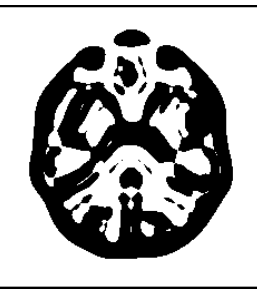

(h) $\tau=080$

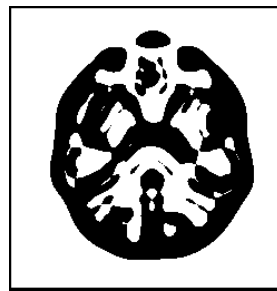

(i) $\tau=090$

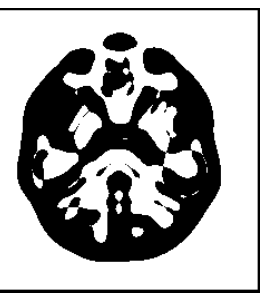

(j) $\tau=100$

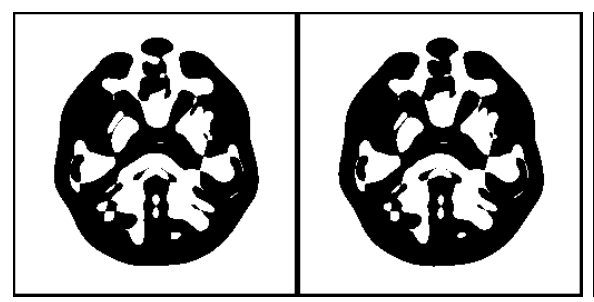

(k) $\tau=110$

(l) $\tau=120$

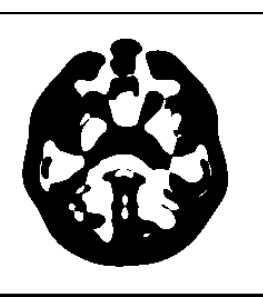

(m) $\tau=130$

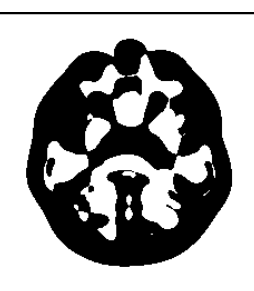

(n) $\tau=140$

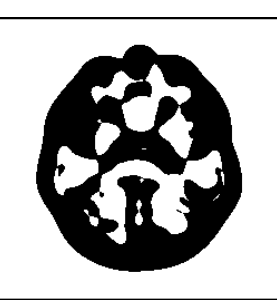

(o) $\tau=150$

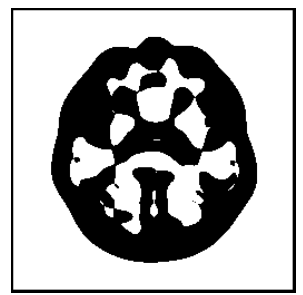

(p) $\tau=160$

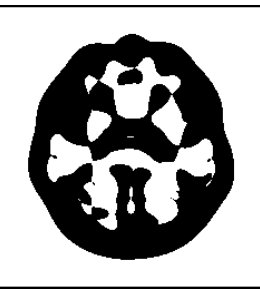

(q) $\tau=170$

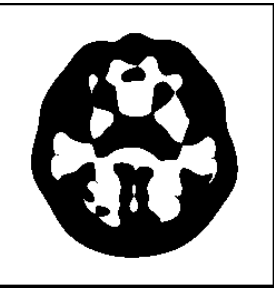

(r) $\tau=180$

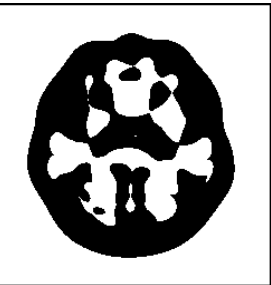

(s) $\tau=190$

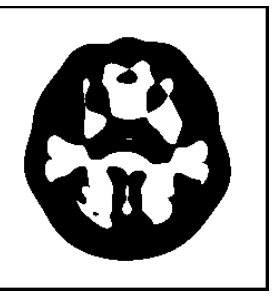

(t) $\tau=200$

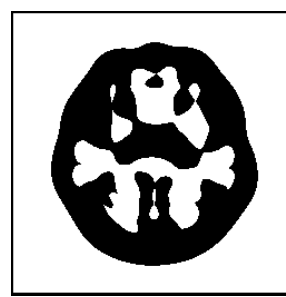

(u) $\tau=210$

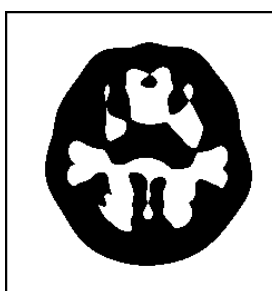

(v) $\tau=220$

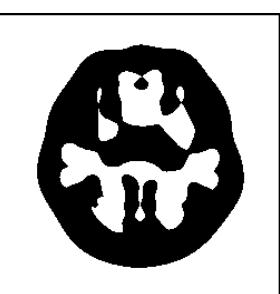

(w) $\tau=230$

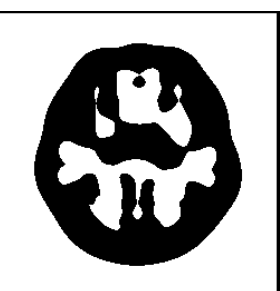

(x) $\tau=240$

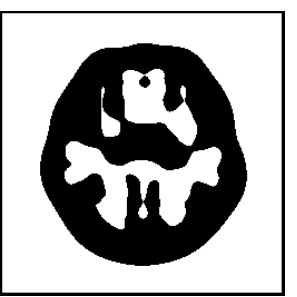

(y) $\tau=250$

Figure 40: Edge lines in the Gaussian scale space. 


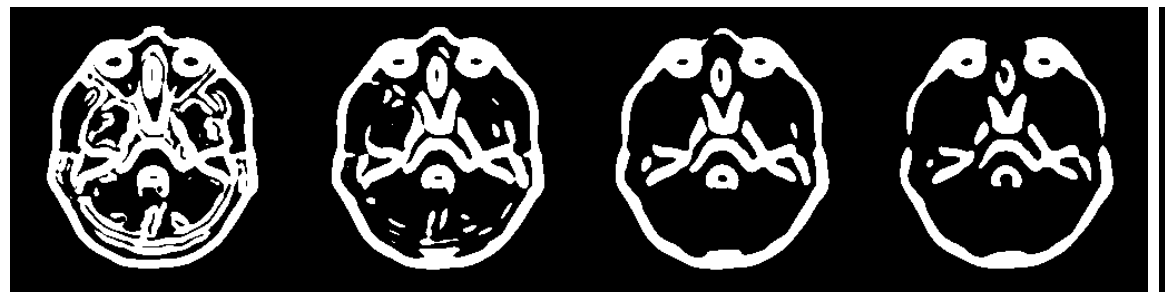

(a) $\tau=010$

(b) $\tau=020$

(c) $\tau=030$

(d) $\tau=040$

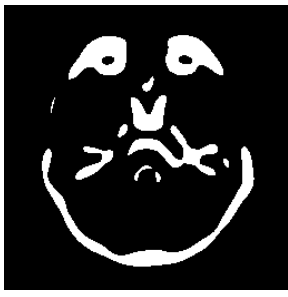

(e) $\tau=050$

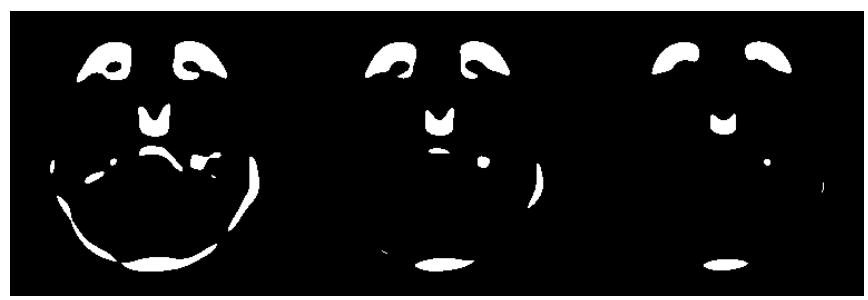

(f) $\tau=060$

(g) $\tau=070$

(h) $\tau=080$

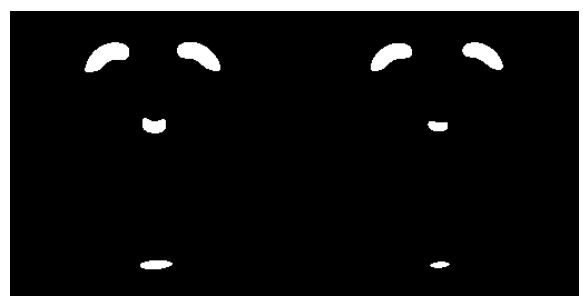

(i) $\tau=090$

(j) $\tau=100$

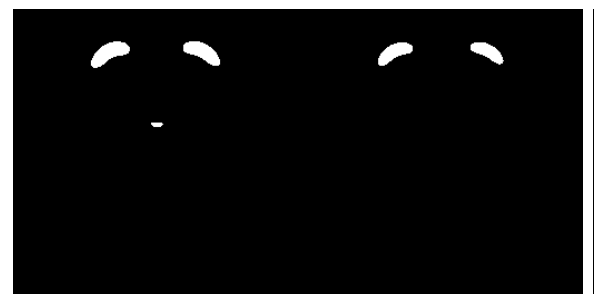

(k) $\tau=110$

(l) $\tau=120$

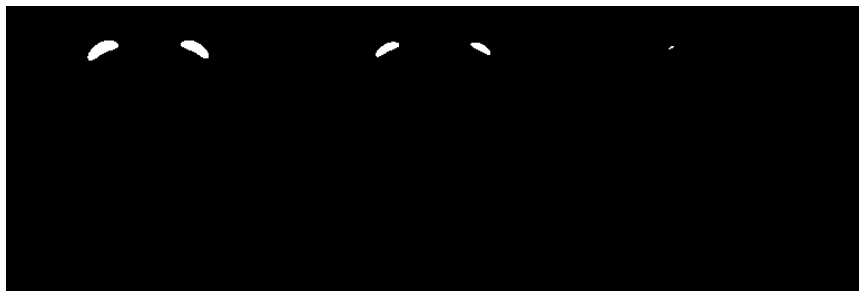

(m) $\tau=130$

(n) $\tau=140$

(o) $\tau=150$

Figure 41: Linear gradient edges, $|\nabla f|>5.0$.

invalid. Segmentation should be employed above such a critical scale.

\subsubsection{Number of Segments in Scale Space}

The number of segments is determined by selecting a scale $\tau$ above the critical scale. All segments are classified into either positive and negative segments at the coarsest scale, which is represented by a pair of subroots of the scale-space tree of image segments. As the scale decreases, new image segments appear one after another. The appearance of such segments indicates that a segment splits into subsegments, which are represented by the nodes of our scale-space tree.

The detection of the critical scale is achieved by observing the decay of the number of segments with respect to the scale. Let us consider the number of segments of an image with random features. If bright dots are uniformly distributed throughout an image, the number of local maxima of $f(\boldsymbol{x}, \tau)$ corresponding to the dots exponentially decays with increasing scale (Roberts, 1997). Therefore, we conjecture that the number of segments approximately shows exponential decay. We assert that the segmentation of such an image with random features yields only invalid segments. Conversely, nonexponential decay of the number of segments implies non-uniformity of the distribution of segments. Exponential decay is allowed only in the small scale where the segment distribution seems to be locally uniform. Statistically, the deterministic features of the image emerge above the critical scale where the monotonic decay collapses.

In practice, the critical scale can be roughly estimated by counting the number of segments at every scale. The critical scale is at the end of the exponential decay of the number of segments. We compare the variation in the number of segments between a random texture image and the same image with deterministic structures as shown in Fig. 44. If there exists valid segments, one can see the number of segments remaining at some value for a wide range of scale above the critical scale. 


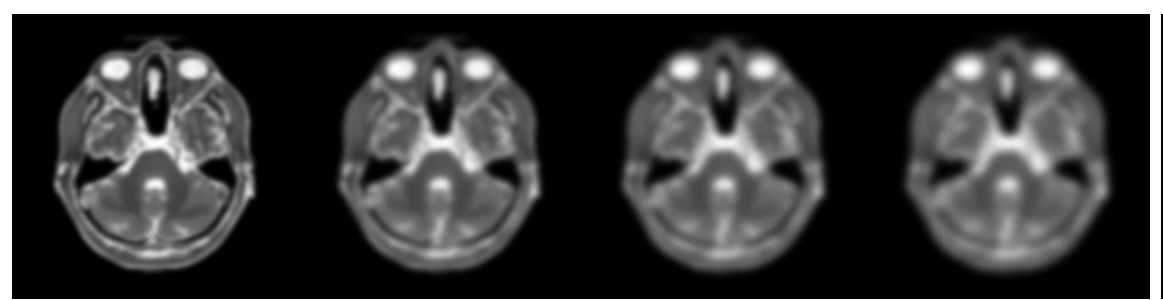

(a) $\tau=010$

(b) $\tau=020$

(c) $\tau=030$

(d) $\tau=040$

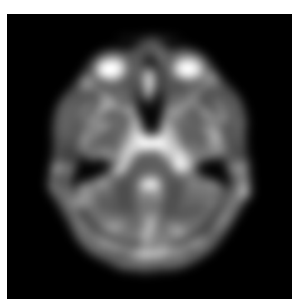

(e) $\tau=050$

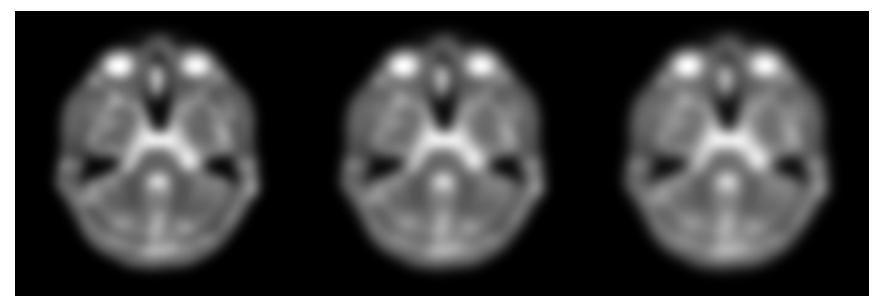

(f) $\tau=060$

(g) $\tau=070$

(h) $\tau=080$

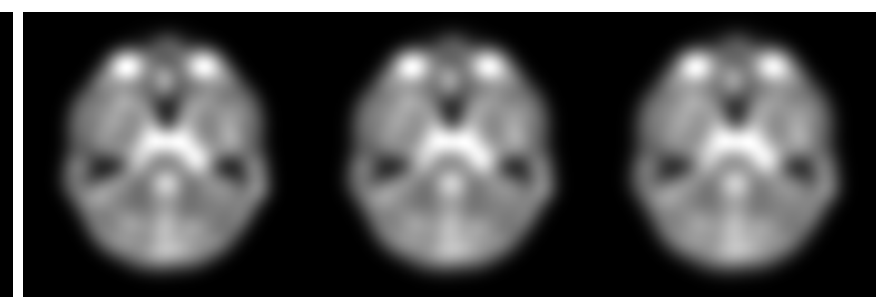

(k) $\tau=110$

(l) $\tau=120$

(m) $\tau=130$

(n) $\tau=140$

(o) $\tau=150$

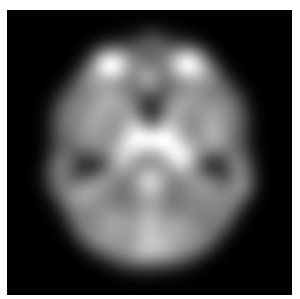

(p) $\tau=160$

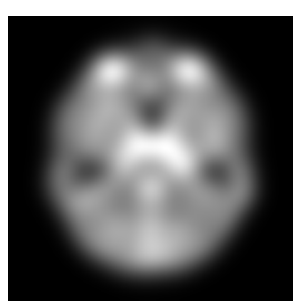

(u) $\tau=210$

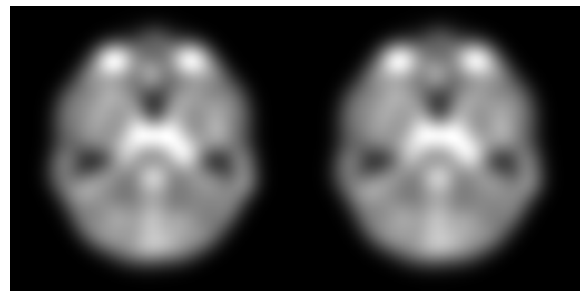

(q) $\tau=170$

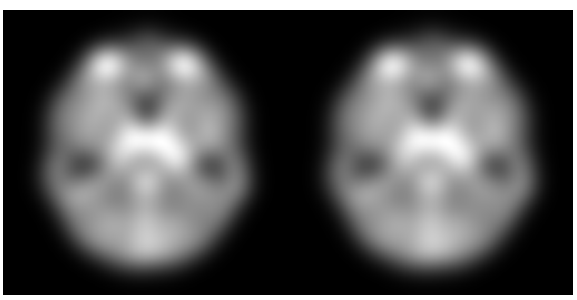

(v) $\tau=220$

(w) $\tau=230$

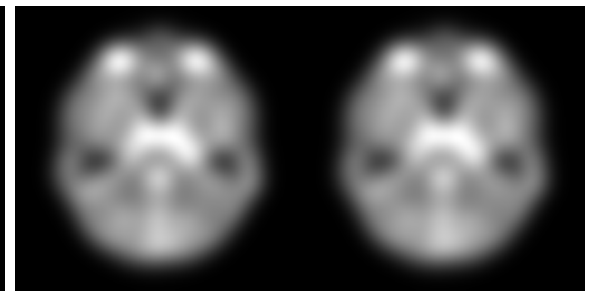

(s) $\tau=190$

(t) $\tau=200$

Figure 42: Linear diffused images. 


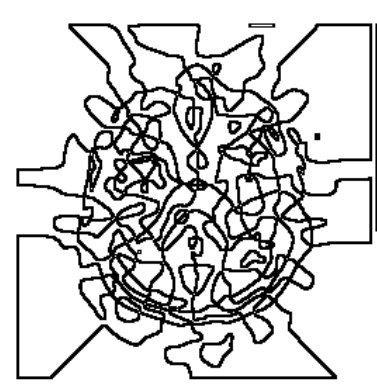

(a) Characteristic line $\tau=30$

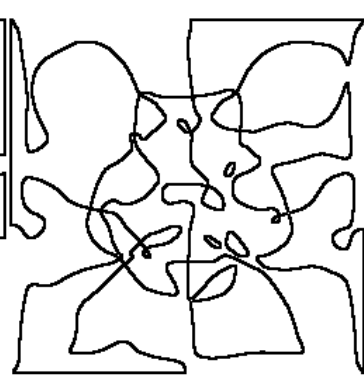

(b) Characteristic line $\tau=360$

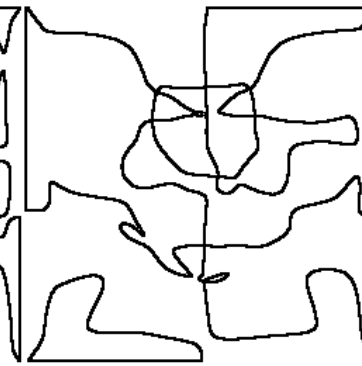

(c) Characteristic line ; $\tau=560$

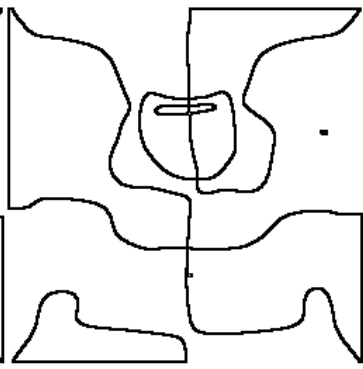

(d) Characteristic line ; $\tau=760$

Figure 43: Characteristic lines in the linear scale space.

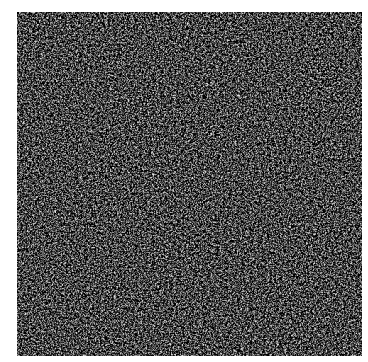

(a)

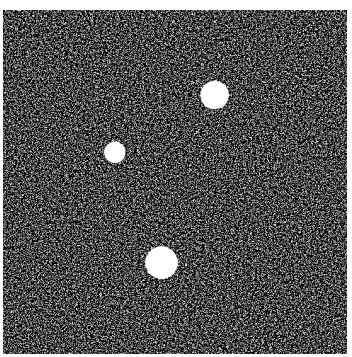

(b)

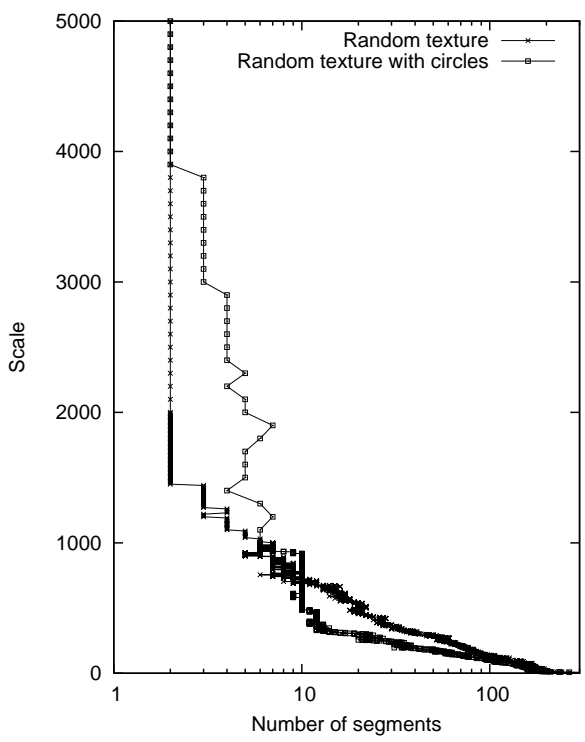

(c)

Figure 44: Decaying forms of the number of segments. (a) Random texture. (b) White circles in the random texture. (c) Number of segments for (a) and (b). The exponential decay collapses at the critical scale, and four valid segments are detected. They correspond to the segments for the circles and the background.

While the decrease in the number of segments is almost exponential for the texture image in Fig. 44(a), several segments remain for a long scale for the image in Fig. 44(b). This indicates that there exist three valid segments in Fig. 44(b). 


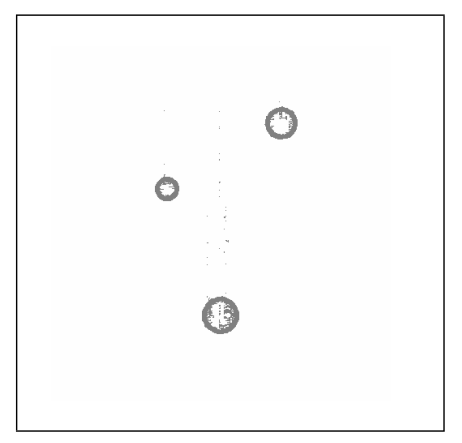

Figure 45: Segmentation results for the image in Fig. 44(b).

\subsubsection{Validation of Segments}

Any image segment in $S_{ \pm}$can be recursively validated using the corresponding subtree of the scalespace tree of segments. Suppose we detect a segment above the critical scale. Then the segment is validated by the decaying form of the number of subsegments represented by the corresponding subtree. If we find the critical scale for the subtree, the segment has valid subsegments. If no critical scale is found, the segment contains only random features such as texture.

Since every pixel of an image corresponds to one of the segments detected above the critical scale, we can partition the image into valid segments according to the scale-space tree of segments. Consequently, an image can be recursively segmented into valid segments, each of which consists of the subsegments represented by the leaves of the corresponding subtree.

We demonstrate our segmentation method on the artifical image in Fig. 44(b). The critical scale is roughly estimated as $\tau_{c}=800$, at which the number of segments is ten. The edges of the three filled circles are correctly detected while the noisy background is identified as a simple large area with some negligible stain among the circles. These spurious segments in the background may be due to the discrete implementation of derivative calculation. This result indicates that the deterministic segments can be distinguished from the stochastic segments by the hierarchical structure across scales, that is, the deep structure.

\section{Scale-Space Analysis of Point Cloud}

\subsection{Estimation of Point Cloud Density}

Let $P=\left\{\boldsymbol{p} \mid \boldsymbol{p} \sim f, \boldsymbol{p} \in \mathbb{R}^{d}\right\}$ be a point cloud in $d$-dimensional Euclidean space. Here, $f(\boldsymbol{x})$ is an unknown probability density function (PDF). The PDF describes the distribution of the relative frequency with which the sample point can be obtained as an element of point cloud $P$ by a finite number of repetitions of observation. Unless the PDF is uniform, it provides a meaningful difference in the frequency with respect to spatial position. Our purpose is to extract informative features of the unknown PDF $f(\boldsymbol{x})$ from the point cloud, since the spatial pattern of the point cloud is governed by the PDF. Point cloud $P$ with a finite cardinality illustrates the density distribution at some level of geometric detail. Therefore, the extraction of the informative features can be interpreted as computation of estimable geometric attributes of $\operatorname{PDF} f(\boldsymbol{x})$ from point cloud $P$.

Stochastic moments are such attributes that quantify the geometric features of the PDF. The moments characterise the distribution shape as a centroid, variance, and distortion (asymmetry by skewness, peakedness by kurtosis, ..), For principal component analysis (PCA), the PDF $f(\boldsymbol{x})$ is assumed to be a $d$-dimensional Gaussian function whose mean vector and covariance matrix define its ellipsoidal equiprobable level set.

The derivatives of $f(\boldsymbol{x})$ also describe the differential geometric features. Local maximisers of $f(\boldsymbol{x})$, or the modes $\{\boldsymbol{\xi} \mid \nabla f(\boldsymbol{\xi})=\mathbf{0}\}$, are the most expected and typical features within a group or a class of the features. We will discuss the behaviour of the modes in scale space, which will give us insight into dimension reduction with respect to the loss of information. The mode behaviour also leads to a natural method of data clustering known as scale-based clustering or scale-space clustering (Chakravarthy and Ghosh, 1996; Roberts, 1997; Nakamura and Kehtarnavaz, 1998; Hinneburg and Keim, 1998; Leung, Zhang, and Xu, 2000). Hereafter, we call $\tilde{f}(\boldsymbol{x}, \sigma)$ the generalised PDF after the 
fashion of scale-space theory (Zhao and Iijima, 1985b).

If we have no a prior belief on parametric form of $f(\boldsymbol{x})$, the nonparametric approach is applicable to the PDF estimation. The nonparametric kernel estimate of $f(\boldsymbol{x})$ (Rosenblatt, 1956; Parzen, 1962) is

$$
\tilde{f}(\boldsymbol{x}, \sigma)=\frac{1}{\operatorname{card}(P)} \sum_{\boldsymbol{p} \in P} K(\boldsymbol{x}-\boldsymbol{p}, \sigma),
$$

where $K$ is the normalised kernel function, and $\sigma$ is called the bandwidth of the kernel. This estimated PDF $\tilde{f}(\boldsymbol{x}, \sigma)$ in Eq. (116) is normalised so that

$$
\int_{\boldsymbol{x} \in \mathbb{R}^{d}} \tilde{f}(\boldsymbol{x}, \sigma) d V=1 .
$$

The estimated PDF $\tilde{f}(\boldsymbol{x}, \sigma)$ with a suitable kernel function converges to the true PDF $f(\boldsymbol{x})$ if $\sigma \rightarrow 0$ when the cardinality of $P$ approaches infinity.

The Gaussian kernel

$$
K(\boldsymbol{x}, \sigma)=G(\boldsymbol{x}, \sigma)=\frac{1}{\sqrt{2 \pi \sigma^{2}} d} e^{-\frac{|\boldsymbol{x}|^{2}}{2 \sigma^{2}}}
$$

has been widely used for the PDF estimation (Izenman, 1991). In the PDF estimation using the Gaussian kernel, the bandwidth $\sigma$ determines how much the point cloud is smoothed to produce the density estimate. Although there is a body of literature dealing with the problem on bandwidth selection in the kernel density estimation (Izenman, 1991), analysis of $\tilde{f}(\boldsymbol{x}, \sigma)$ in $(\boldsymbol{x}, \sigma)$-space is of great help to clarify how the structure of the point cloud is simplified with increasing $\sigma$. The Gaussian scale space (Witkin, 1983; Koenderink, 1984; Weickert, Ishikawa, and Imiya, 1999; Lindeberg, 1994) can be applicable to the PDF estimation generating $\tilde{f}(\boldsymbol{x}, \sigma)$ using the Gaussian kernel as a scalespace representation of PDF estimated from the point cloud $P$. The scale parameter $\sigma$ controls the level of estimated geometric detail. We enumerate some properties of the PDF estimation with an isotropic Gaussian kernel.

- $\tilde{f}(\boldsymbol{x}, \sigma)$ satisfies the scale-space axioms (Witkin, 1983; Weickert, Ishikawa, and Imiya, 1999; Duits, Florack, and J. Graaf, 2004), which include invariance under basic geometric transforms.

- Setting $\sigma=\sqrt{2 \tau}, \tilde{f}(\boldsymbol{x}, \sqrt{2 \tau})$ satisfies the linear diffusion equation

$$
\frac{\partial \tilde{f}}{\partial \tau}=\Delta \tilde{f}
$$

The initial function at $\tau=0$ is the $\delta$-mixture in Eq. (120), and a superposition of the Gaussian functions represents uncertainty of the location of the points after time $\tau$.

- $f(\boldsymbol{x}, \sigma)$ converges to a mixture of the Dirac's delta function as $\sigma \rightarrow 0$.

$$
\lim _{\sigma \rightarrow 0} \tilde{f}(\boldsymbol{x}, \sigma)=\frac{1}{\operatorname{card}(P)} \sum_{\boldsymbol{p} \in P} \delta(\boldsymbol{x}-\boldsymbol{p})
$$

In other words, $f\left(\boldsymbol{x}, 0_{+}\right)$acts as a lookup table because it returns $\infty$ only at the data points $\boldsymbol{x}=\boldsymbol{p}$ and otherwise is 0 .

- In the limit of $\sigma \rightarrow \infty$, the function $\tilde{f}(\boldsymbol{x}, \sigma)$ converges to zero with the volume of one. Such a PDF is said to be featureless providing null information.

- The number of modes of the homoscedastic Gaussian mixture seldom increases as the scale $\tau$ increases (Carreira-Perpinan and Williams, 2003). That is, mode creation is less expected if the Gaussian functions are unequally weighted. It is known that anisotropic Gaussian mixtures with different covariances yield spurious modes outside the convex hull of $P$.

- The scale parameter $\sigma$ controls the information quantity measured by Shannon entropy (Sporring and Weickert, 1999), that is, the measure of uncertainty monotonically increases with $\sigma$. 


\subsection{Behaviour of Modes in Scale Space}

One of the primitive geometric features of the generalised PDF is the stationary point (a.k.a. critical point) where the spatial gradient vanishes.

$$
\{(\boldsymbol{\xi}, \sigma) \mid \nabla \tilde{f}(\boldsymbol{\xi}, \sigma)=\mathbf{0}\}
$$

The stationary point can be classified into $d+1$ types based on the combination of signs of the eigenvalues $\lambda_{l}$ of the Hessian matrix $\boldsymbol{H}=\nabla \nabla^{\top} f(\boldsymbol{x}, \sigma)$. We denote the signs of the eigenvalues as $( \pm, \pm, \ldots, \pm)$.

Trajectory of Mode The position of the stationary point changes with respect to the scale. The trajectory of the stationary point in the scale space is called the stationary curve (a.k.a. critical curve) in the scale-space theory. A stationary curve can be denoted by the one-dimensional manifold $\boldsymbol{\xi}(\sigma)$ in the Gaussian scale space. Based on Zhao and Iijima (Zhao and Iijima, 1985a), the stationary curve is the solution of the system of differential equations

$$
\boldsymbol{H} \frac{d \boldsymbol{\xi}(\sqrt{2 \tau})}{d \boldsymbol{\tau}}=-\nabla \Delta \tilde{f}(\boldsymbol{\xi}, \sqrt{2 \tau}) .
$$

The trajectory of a mode of $\tilde{f}(\boldsymbol{x}, \sigma)$ also satisfies Eq. (122). Every point $(\boldsymbol{x}, \sigma)=(\boldsymbol{p}, 0)(\boldsymbol{p} \in P)$ is a starting point of the trajectory of the mode. The trajectory of the mode has an endpoint in scale space. In this study, we denote the scale of the endpoint as $\sigma^{\mathrm{t}}$.

Equiprobable Level Set The probability density at the mode, i.e., $\tilde{f}(\boldsymbol{\xi}, \sigma)$, must decrease with increasing $\sigma$ since $\tilde{f}(\boldsymbol{x}, \sigma)$ obeys Eq. (119) and Laplacian $\Delta \tilde{f}=\operatorname{trace} \boldsymbol{H}=\sum_{l} \lambda_{l}<0$ at the mode. This indicates that equiprobable level sets are nested in the scale space. In image analysis, the nested level set associated with a local extremum is called the extremum stack (Lifshitz and Pizer, 1990; Simmons, Arridge, Tofts, and Barker, 1998). In the same manner, one can associate a mode $\boldsymbol{\xi}\left(\sigma_{0}\right)$ with an equiprobable level set in scale space with a probability density equal to $\tilde{f}\left(\boldsymbol{\xi}, \sigma_{0}\right)$.

Flow of Probability Density As the scale $\sigma$ increases, the probability density disperses in $\mathbb{R}^{d}$ space maintaining the normalised condition in Eq. (117). Since Eq. (119) governs this process, the dispersing flow $\boldsymbol{F}$ of probability density can be defined as

$$
\boldsymbol{F}=-\nabla \tilde{f}(\boldsymbol{x}, \sigma) .
$$

The local maxima, minima and saddles are sources, drains, and confluent points of density flow with respect to the scale (Zhao and Iijima, 1985b; Lindenbaum, Fischer, and Bruckstein, 1994; Sakai and Imiya, 2005a). A separatrix of the inward flow curves connects between the modes of $\tilde{f}(\boldsymbol{x}, \sigma)$, that is, the sources of the flow.

In higher dimensional spaces, the separatrices are hypersurfaces which separate regions of different flow behaviour. We have $d-1$ types of saddles in a $d$-dimensional space. Let us denote the numbers of positive and negative eigenvalues of $\boldsymbol{H}$ at a stationary point by $s_{+}$and $s_{-}$, respectively. Then, the space in the vicinity of the saddle can be decomposed into $s_{+}$-dimensional and $s_{-}$-dimensional subspaces, $S_{+}$and $S_{-}$, each of which is spanned by the corresponding eigenvectors. Since the saddle in $\mathbb{R}^{d}$ is a local minimum in the subspace $S_{+}$, the density flow in $S_{+}$is in inward directions to the saddle. A similar statement holds for $S_{-}$. Therefore, $S_{+}$and $S_{-}$can be called the subspaces of the attracting separatrix and the repelling separatrix of a saddle, respectively. We note that the stationary points of $\tilde{f}(\boldsymbol{x}, \sigma)$ are representative points of geometric components, which can be symbolised as a graph of the flow-curve connections. In three-dimensional space, for example, local maxima $(-,-,-)$ correspond to vertices of the graph. Saddles $(+,-,-)$ and $(+,+,-)$ represent edges and faces of the graph, respectively. Local minima reside in volumes. See Fig. 46(a).

Structural Simplification If the scale $\sigma$ is sufficiently small, the generalised $\operatorname{PDF} \tilde{f}(\boldsymbol{x}, \sigma)$ consists of card $(P)$ small blobs in an isotropic Gaussian shape. As $\sigma$ increases, the blobs merge with each other into large ones, and the modes at their peaks disappear one after another. The topological structure formed by the flow-curve connections is simplified according to this degeneration of $\tilde{f}(\boldsymbol{x}, \sigma)$.

It is known from scale-space theory and catastrophe theory that the fold catastrophe generically describes annihilation and creation events of two stationary points, which differ with respect to 
the sign of one eigenvalue of $\boldsymbol{H}$ that becomes zero at the point of events (Kuijper, Florack, and Viergever, 2003; Kuijper and Florack, 2005). Therefore, a mode of $\tilde{f}(\boldsymbol{x}, \sigma)$ with signs $(-,-, \ldots,-)$ is generically annihilated with a saddle with $\left(s_{+}, s_{-}\right)=(1, d-1)$. Similarly, two saddles with $\left(s_{+}, s_{-}\right)$ and $\left(s_{+}+1, s_{-}-1\right)$ (or $\left.\left(s_{+}-1, s_{-}+1\right)\right)$ meet and disappear at a point in the scale space. Saddles with $\left(s_{+}, s_{-}\right)=(d-1,1)$ can be annihilated with local minima with $\left(s_{+}, s_{-}\right)=(d, 0)$. A point at infinity in the scale space as one of the local minima for topological consistency (Sakai and Imiya, 2005a, 2007). Equation (122) shows that the motion of the two points just before annihilation is in the direction of the zero principal curvature (Sakai and Imiya, 2005b, 2007).

As a consequence, every type of stationary point is involved in the sequence of the simplification of topological structure. Figure 46 illustrates an example of the structural simplification in a threedimensional space. Observe how the dimensionality is reduced from three to zero when the modes disappear. Even in higher dimensional spaces, one can find a subspace spanned only by the stationary points involved in the structural simplification, and observe a similar process of dimension reduction.

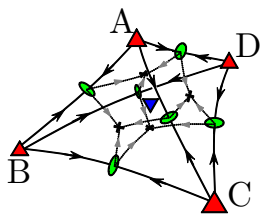

(a)

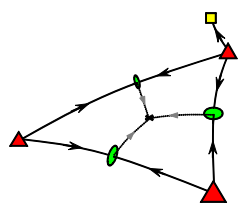

(e)

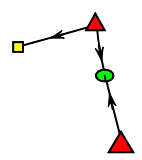

(i)

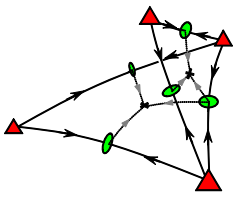

(b)

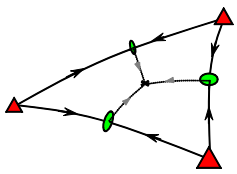

(f)

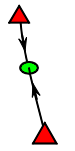

(j)

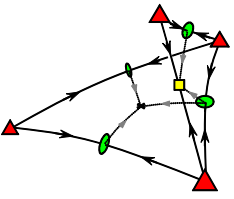

(c)

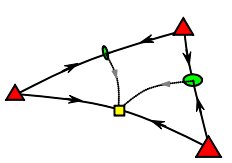

(g)

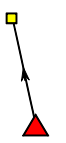

(k)

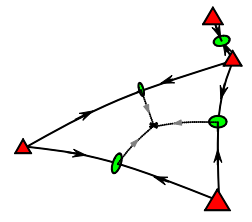

(d)

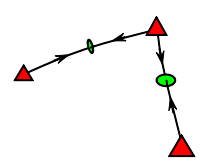

(h)

Figure 46: Example of the simplification of topological structure of generalised PDF. The upward and downward triangles indicate local maxima (mode) and local minima. The discs and crosses indicate the saddles with signs $(+,-,-)$ and $(+,+,-)$, respectively. The solid lines are the flowcurve connections associated with the $(+,-,-)$ saddles. The dotted lines are the connections between the of $(+,-,-)$ and $(+,+,-)$ saddles. The structure is simplified from (a) to (l). At the scale of annihilation, the annihilation point is indicated by the square.

Convergence to Centroid If $\sigma$ is sufficiently larger than the spatial size of the point cloud, the whole point cloud is regarded as a universal cluster represented by one remaining mode of the generalised PDF. This mode converges to the centroid of the point cloud according to the following proposition in (Zhao and Iijima, 1985b; Loog, Duistermaat, and Florack, 2001).

Proposition 4 One remaining local maximum $\boldsymbol{\xi}(\sigma)$ of $\tilde{u}(\boldsymbol{x}, \sigma)=G(\boldsymbol{x}, \sigma) * u(\boldsymbol{x})$, that is, a convolution function with the Gaussian, converges to the centroid of $u(\boldsymbol{x})$ if $\sigma \rightarrow \infty$.

\section{Proof 5}

$$
\begin{aligned}
\nabla \tilde{u}(\boldsymbol{x}, \sigma) & =\nabla(G * u)=(\nabla G) * u \\
& =(\boldsymbol{x} G) * u-\boldsymbol{x}(G * u) .
\end{aligned}
$$

Since $\nabla \tilde{u}=\mathbf{0}$ at the local maximum $\boldsymbol{\xi}(\sigma)$, we have

$$
\boldsymbol{\xi}(\sigma)=\frac{(\boldsymbol{x} G) * u}{G * u} \underset{55}{\rightarrow} \frac{\boldsymbol{x} * u}{1 * u} \quad(\sigma \rightarrow \infty) .
$$


The generalised PDF $\tilde{f}(\boldsymbol{x}, \sigma)$ can be described as a Gaussian convolution of the delta mixture in Eq. (120). The centroid of a PDF is nothing more than the mean vector, that is, the first moment.

Mode Hierarchy The generalised PDF $\tilde{f}(\boldsymbol{x}, \sigma)$ starts with card $(P)$ Gaussian blobs. The merging process of the blobs with respect to scale hierarchically associates the modes of blobs with each other. Thus, the $\operatorname{card}(P)$ points are classified into hierarchical clusters. The hierarchy among modes is described as a tree, which is called the mode tree (Minnotte and Scott, 1993). The mode tree also represents the hierarchy of the points or clusters in the point cloud. Note that not only the modes but also all of the other types of stationary points have hierarchical relationships among them. The mode tree is a subgraph of the scale-space tree for local maxima (Sakai and Imiya, 2005b).

\subsection{Hierarchical Clustering and Validation}

\subsubsection{Scale-Based Hierarchical Clustering}

Clustering methods of data points using scale space have been proposed by many authors (Chakravarthy and Ghosh, 1996; Roberts, 1997; Nakamura and Kehtarnavaz, 1998; Hinneburg and Keim, 1998; Kothari and Pitts, 1999; Leung, Zhang, and Xu, 2000; Sakai, Imiya, Komazaki, and Hama, 2007). Most of them can be considered to be based on the mode hierarchy described in the previous section. We have presented an algorithm of the construction of a mode tree for hierarchical clustering (Sakai, Imiya, Komazaki, and Hama, 2007).

The detected clusters, however, are invalid at small scales. The smaller the scale is, the more the modes of $\tilde{f}(\boldsymbol{x}, \sigma)$ are dependent on the positions of sample points. If point cloud $P$ does not have sufficient cardinality, the generalised $\operatorname{PDF} \tilde{f}(\boldsymbol{x}, \sigma)$ cannot approximate the true $\operatorname{PDF} f(\boldsymbol{x})$ in detail at the small scales. As a result, any estimate using the generalised PDF $\tilde{f}(\boldsymbol{x}, \sigma)$ with small scales is highly random and experimentally less reproducible. We require a validation scheme to identify the clusters by modes with statistically significant reproducibility.

The detected clusters in scale space have been validated by several properties of the clusters: the number of clusters vs. scale, compactness, isolation, lifetime and birthtime (Roberts, 1997; Nakamura and Kehtarnavaz, 1998; Hinneburg and Keim, 1998; Leung, Zhang, and Xu, 2000). It is suggested that the decrease in the total number of clusters pauses at the number of valid clusters for a relatively long period of the scale. Thier methods of finding such pauses, however, are heuristic.

\subsubsection{Cluster Validation by Critical Scale}

We have proposed a statistical criterion to identify the valid clusters using the life of the mode, which is defined as the terminating scale of the trajectory of the mode in scale space, that is, the life is expressed by $\sigma^{\mathrm{t}}$. In ref. (Sakai, Imiya, Komazaki, and Hama, 2007), we focused on the axiomatic fact that a set of uniformly distributed points does not contain the reproducible clusters. Furthermore, we showed that the uniformly distributed points present a Weibull-like unimodal distribution of the life. The valid cluster can be defined as a cluster with a statistically significant life out of this unimodal distribution. Consequently, the cluster validation can be established by the statistical rejection method using the unimodal life distribution (Sakai, Imiya, Komazaki, and Hama, 2007).

We call the critical value of the scale used for the rejection the critical scale. Since any estimate using $\tilde{f}(\boldsymbol{x}, \sigma)$ with scales smaller than the critical scale is judged to be invalid, the critical scale is a threshold of spatial measure above which the given data is informative and under which any result of pattern analyses using the PDF estimation looses statistical significance. In the scale-based clustering, the critical scale is the significance level of cluster validity. The critical scale also provides the statistical significance in the determination of the dimensionalities of subspaces of clusters, which will be discussed in the next section. The following algorithm recursively discovers valid clusters using the mode tree.

ClusterDiscovery (mode tree $T$, set of clusters $U$, critical value $\alpha$ )

1 let $\Sigma$ be the set of life values stored in $T$ except the root;

2 let $s$ be the subroot node of $T$ with the largest life value $\sigma_{\max } \in \Sigma$;

3 if IsRejected $\left(\sigma_{\max }, \Sigma, \alpha\right)$

4 ClusterDiscovery $(\operatorname{Subtree}(T, s), U, \alpha)$;

$5 \quad$ ClusterDiscovery $(T \backslash \operatorname{Subtree}(T, s), U, \alpha)$; 
6 else push $C:=\operatorname{DataPoints}(T)$ into $U$;

7 endif.

Here, IsRejected is the function that performs the rejection and returns true if $\sigma_{\max }$ is significantly large for the given critical value $\alpha$. The function Subtree extracts the subtree with subroot $s$ from the tree $T$. DataPoints returns a set of data points with labels that are recorded at the nodes in the given tree.

\subsection{Mathematical Framework of Scale-Based PCA}

\subsubsection{Stochastic Moment}

In probability theory and statistics, structural features of data distribution are typically described by the stochastic moments. For a given PDF $f(\boldsymbol{x})$, the $n$ th-order stochastic moment is given by

$$
I_{n}:\left.f \rightarrow \frac{1}{i^{n}} \nabla_{\boldsymbol{k}}^{n} \phi(\boldsymbol{k})\right|_{\boldsymbol{k}=\mathbf{0}}
$$

Here, $i=\sqrt{-1}$, and $\phi$ denotes the characteristic function defined as

$$
\phi(\boldsymbol{k})=\mathcal{F}[f(\boldsymbol{x})](\boldsymbol{k})=\int_{\boldsymbol{x} \in \mathbb{R}^{d}} f(\boldsymbol{x}) e^{i \boldsymbol{k}^{\top} \boldsymbol{x}} d V,
$$

which is similar to the moment-generating function $M(\boldsymbol{t})=\phi(-i \boldsymbol{t})$. For example, $I_{1}$ and $I_{2}$

$$
\begin{aligned}
& I_{1} \quad: \quad f \rightarrow \int_{\boldsymbol{x} \in \mathbb{R}^{d}} \boldsymbol{x} f(\boldsymbol{x}) d V=E[\boldsymbol{x}]=\boldsymbol{\mu} \\
& I_{2} \quad: \quad f \rightarrow \int_{\boldsymbol{x} \in \mathbb{R}^{d}} \boldsymbol{x} \boldsymbol{x}^{\top} f(\boldsymbol{x}) d V=E\left[\boldsymbol{x} \boldsymbol{x}^{\top}\right]=\boldsymbol{M}
\end{aligned}
$$

are, respectively, mappings from a $\operatorname{PDF} f(\boldsymbol{x})$ to the mean vector and the moment matrix with respect to the origin.

\subsubsection{Central Moment}

The stochastic moment can be converted to a moment about a specific point. We modify $I_{n}$ to define the stochastic moment with respect to $\boldsymbol{a} \in \mathbb{R}^{d}$ as

$$
I_{n}(\boldsymbol{a}):\left.f \rightarrow \frac{1}{i^{n}} \nabla_{\boldsymbol{k}}^{n} \phi(\boldsymbol{k}) e^{-i \boldsymbol{k}^{\top} \boldsymbol{a}}\right|_{\boldsymbol{k}=\mathbf{0}}
$$

Clearly, $I_{n}(\mathbf{0})$ is equivalent to $I_{n} . I_{n}(\boldsymbol{a})$ defines the translation of the stochastic moment. The characteristic function can be locally expanded in power series as

$$
\begin{aligned}
\phi(\boldsymbol{k}) e^{-i \boldsymbol{k}^{\top} \boldsymbol{a}}= & \int_{\boldsymbol{x} \in \mathbb{R}^{d}} f(\boldsymbol{x}) e^{i \boldsymbol{k}^{\top}(\boldsymbol{x}-\boldsymbol{a})} d V \\
= & \int_{\boldsymbol{x} \in \mathbb{R}^{d}} f(\boldsymbol{x}) \sum_{m=0}^{\infty} \frac{1}{m !}\left\{i \boldsymbol{k}^{\top}(\boldsymbol{x}-\boldsymbol{a})\right\}^{m} d V \\
= & \int_{\boldsymbol{x} \in \mathbb{R}^{d}} f(\boldsymbol{x}) d V+i \int_{\boldsymbol{x} \in \mathbb{R}^{d}} \boldsymbol{k}^{\top}(\boldsymbol{x}-\boldsymbol{a}) f(\boldsymbol{x}) d V \\
& +\frac{1}{2} i^{2} \int_{\boldsymbol{x} \in \mathbb{R}^{d}}(\boldsymbol{x}-\boldsymbol{a})^{\top} \boldsymbol{k} \boldsymbol{k}^{\top}(\boldsymbol{x}-\boldsymbol{a}) f(\boldsymbol{x}) d V \\
& +\cdots
\end{aligned}
$$

As a special case, setting $\boldsymbol{a}=\boldsymbol{\mu}$, we obtain the central moments. For instance, $I_{1}(\boldsymbol{\mu})$ always maps $f$ to zero vector, and $I_{2}(\boldsymbol{\mu})$ yields the covariance matrix.

$$
\begin{aligned}
I_{2}(\boldsymbol{\mu}): f \rightarrow & \int_{\boldsymbol{x} \in \mathbb{R}^{d}}(\boldsymbol{x}-\boldsymbol{\mu})(\boldsymbol{x}-\boldsymbol{\mu})^{\top} f(\boldsymbol{x}) d V \\
& =E\left[\left(\boldsymbol{x}_{57}-\boldsymbol{\mu}\right)(\boldsymbol{x}-\boldsymbol{\mu})^{\top}\right]=\boldsymbol{\Sigma}
\end{aligned}
$$


Therefore, it is possible to regard $I_{n}(\boldsymbol{\mu})$ as the central moment generator. The central moments correspond to the Taylor expansion coefficients of the Fourier transform of the characteristic function (Papoulis, 1962, 1965) about the mean $\boldsymbol{\mu}$. The PDF $f(\boldsymbol{x})$ can be reconstructed from the inverse Fourier transform of its characteristic function if all of the stochastic moments are finite and the series in Eq. (129) converges absolutely near $\boldsymbol{x}=\boldsymbol{a}$.

\subsubsection{Moment Estimation}

The central moments can be derived from any PDF model. If a single $d$-dimensional Gaussian distribution is assumed as the PDF model for a point cloud, that is, $\boldsymbol{p} \sim \mathcal{N}(\boldsymbol{\mu}, \boldsymbol{\Sigma})$, then one can obtain the mean vector $\boldsymbol{\mu}$ and the covariance matrix $\boldsymbol{\Sigma}$ by $I_{1}(\mathbf{0})$ and $I_{2}(\boldsymbol{\mu})$, respectively. One should note that odd-order central moment generators for the Gaussian distribution provide zeros due to the symmetry of the Gaussian function, and any even-order central moment can be expressed as the second-order central moment $\boldsymbol{\Sigma}$. The central moment generator $I_{n}(\boldsymbol{\mu})$ for $n \geq 3$ therefore provides no additional information about the distribution. In fact, PCA can only deal with a linear subspace under the assumption of the multidimensional Gaussian distribution. PCA estimates the major axes of the ellipsoidal equiprobable level set, which is determined by $\boldsymbol{\Sigma}$. Usually, the maximum-likelihood estimates of $\boldsymbol{\mu}$ and $\boldsymbol{\Sigma}$, written as

$$
\begin{aligned}
\boldsymbol{\mu}^{\mathrm{ML}} & =\frac{1}{\operatorname{card}(P)} \sum_{\boldsymbol{p} \in P} \boldsymbol{p} \\
\boldsymbol{\Sigma}^{\mathrm{ML}} & =\frac{1}{\operatorname{card}(P)} \sum_{\boldsymbol{p} \in P}\left(\boldsymbol{p}-\boldsymbol{\mu}^{\mathrm{ML}}\right)\left(\boldsymbol{p}-\boldsymbol{\mu}^{\mathrm{ML}}\right)^{\top}
\end{aligned}
$$

are used in PCA. They are called sample moments.

Henceforth, we derive the central moments from the generalised $\operatorname{PDF} \tilde{f}(\boldsymbol{x}, \sigma)$. We define the characteristic function with scale $\sigma$ as

$$
\begin{aligned}
\tilde{\phi}(\boldsymbol{k}, \sigma) & =\mathcal{F}[\tilde{f}(\boldsymbol{x}, \sigma)] \\
& =\frac{1}{\operatorname{card}(P)} \sum_{\boldsymbol{p} \in P} e^{-\frac{\mid \boldsymbol{k}^{2}}{2 \frac{1}{\sigma^{2}}}} e^{i \boldsymbol{k}^{\top} \boldsymbol{p}} .
\end{aligned}
$$

The mean vector and central moment are then

$$
\begin{aligned}
& I_{1}(\mathbf{0}): \quad \tilde{f} \rightarrow \frac{1}{\operatorname{card}(P)} \sum_{\boldsymbol{p} \in P} \boldsymbol{p}=\tilde{\boldsymbol{\mu}} \\
& I_{n}(\tilde{\boldsymbol{\mu}}) \quad: \quad \tilde{f} \rightarrow \frac{-i^{n}}{\operatorname{card}(P)} \nabla_{\boldsymbol{k}^{n}}^{n} e^{-\frac{\mid \boldsymbol{k}^{2}}{2 \frac{1}{\sigma^{2}}}} \sum_{\boldsymbol{p} \in P} e^{i \boldsymbol{k}^{\top}(\boldsymbol{p}-\tilde{\boldsymbol{\mu}})}
\end{aligned}
$$

The mean vector $\tilde{\boldsymbol{\mu}}$ of the generalised PDF coincides with $\boldsymbol{\mu}^{\mathrm{ML}}$. While $\tilde{\boldsymbol{\mu}}$ is independent of the scale $\sigma, I_{n}(\tilde{\boldsymbol{\mu}})$ generates the central moment as a function of $\sigma$. The covariance matrix $\left.\boldsymbol{\Sigma} \tilde{(} \sigma\right)$ of the generalised PDF is given by $I_{2}(\tilde{\boldsymbol{\mu}})$ as

$$
\begin{aligned}
I_{2}(\tilde{\boldsymbol{\mu}}): \quad \tilde{f} & \rightarrow \frac{1}{\operatorname{card}(P)} \sum_{\boldsymbol{p} \in P}(\boldsymbol{p}-\tilde{\boldsymbol{\mu}})(\boldsymbol{p}-\tilde{\boldsymbol{\mu}})^{\top}+\sigma^{2} \boldsymbol{I} \\
& \left.=\boldsymbol{\Sigma}^{\mathrm{ML}}+\sigma^{2} \boldsymbol{I}=\boldsymbol{\Sigma} \tilde{(} \sigma\right),
\end{aligned}
$$

where $\boldsymbol{I}$ denotes the identity matrix. The scale $\sigma$ increases the diagonal dominance of $\sigma^{2}$. Accordingly, every eigenvalue of the matrix $\boldsymbol{\Sigma} \tilde{(} \sigma)$ is incremented by $\sigma^{2}$ while the eigenvectors of $\left.\boldsymbol{\Sigma} \tilde{(} \sigma\right)$ are equal to those of $\boldsymbol{\Sigma}^{\mathrm{ML}}$.

The matrix $\boldsymbol{\Sigma}(0)$ coincides with $\boldsymbol{\Sigma}^{\mathrm{ML}}$. This property might seem confusing because the generalised PDF with $\sigma=0$ is not Gaussian but a $\delta$-mixture. This is caused by the difference in the PDF model. Unlike the single Gaussian distribution assumed in PCA, the generalised PDF $\tilde{f}(\boldsymbol{x}, \sigma)$ approximates any distribution above a critical scale. If we can select a suitable scale $\sigma$, the scalebased central moments we have derived can quantify the structural features of distribution, such as the principal directions, asymmetry, and peakedness. 


\subsubsection{Scale-Based PCA}

We apply the moment estimation for each valid cluster discovered in the point cloud $P$ by the algorithm ClusterDiscovery. According to the mode tree, the generalised PDF $\tilde{f}(\boldsymbol{x}, \sigma)$ can be hierarchically decomposed into the PDFs for the valid clusters.

$$
\tilde{f}(\boldsymbol{x}, \sigma)=\sum_{c=1}^{C} \tilde{f}_{c}(\boldsymbol{x}, \sigma)=\sum_{c=1}^{C} \sum_{\boldsymbol{p} \in P_{c}} G(\boldsymbol{x}-\boldsymbol{p}, \sigma)
$$

Here, $P_{c}(c=1, \ldots, C)$ are the valid clusters corresponding to the subtrees in the mode tree, and $P=\cup_{c} P_{c}$. We note that the scale $\sigma$ in Eq. (136) is merely a common parameter controlling the whole scale of the PDF. There exists a suitable scale $\sigma_{c}$ for each $\tilde{f}_{c}$ to describe the distribution of the $c$-th cluster $P_{c}$. Such $\sigma_{c}$ should at least be greater than the critical scale so as for the cluster $P_{c}$ to be valid, and less than the life $\sigma^{\mathrm{t}}$ for $P_{c}$ to be separate from the others.

Let $\tilde{\boldsymbol{\mu}}_{c}$ and $\left.\boldsymbol{\Sigma}_{c} \tilde{(} \sigma_{c}\right)$ denote the mean vector and covariance matrix of $P_{c}$ calculated by $I_{1}(\mathbf{0})$ in Eq. (133) and $I_{2}\left(\tilde{\boldsymbol{\mu}}_{c}\right)$ in Eq. (135) using $\tilde{f}_{c}\left(\boldsymbol{x}, \sigma_{c}\right)$, respectively. Then, we can employ PCA for the cluster $P_{c}$ by eigendecomposition of $\boldsymbol{\Sigma}_{c}\left(\sigma_{c}\right)$. Since the scale-based covariance matrix is written as

$$
\left.\boldsymbol{\Sigma}_{c} \tilde{(} \sigma_{c}\right)=\boldsymbol{\Sigma}_{c}^{\mathrm{ML}}+\sigma_{c}^{2} \boldsymbol{I},
$$

the eigenvectors of $\left.\boldsymbol{\Sigma}_{c} \tilde{(} \sigma_{c}\right)$ are same as those of $\boldsymbol{\Sigma}_{c}^{\mathrm{ML}}$. The eigenvalues of $\left.\boldsymbol{\Sigma}_{c} \tilde{(} \sigma_{c}\right)$ are greater than or equal to $\sigma_{c}^{2}$. This simple contribution of the scale parameter to the eigenvalues suggests that the eigenvalues of $\boldsymbol{\Sigma}_{c}^{\mathrm{ML}}$ less than the square of critical scale are buried under the scale contribution $\sigma_{c}^{2}$. Such small eigenvalues are neither principal nor statistically significant.

The selection of the number of principal components in PCA is essentially the same as detection of the dimensionality of the subspace of data. Equation. (137) provides us with a statistically reasonable criterion: if the cluster is discovered by the rejection method with a critical scale $\sigma_{\alpha}$, choose the eigenvalues of $\boldsymbol{\Sigma}_{c}^{\mathrm{ML}}$ which are greater than $\sigma_{\alpha}^{2}$.

\subsubsection{Precision Cardinality}

Algorithm ClusterDiscovery can be modified to find point correspondences for two or more sets of points. Given $N$ point sets $P_{n}(n=1, \ldots, N)$, we obtain a set of subtrees of the scale-space tree for the point set $P=\bigcup_{n=1}^{N} P_{n}$ as follows.

\section{PointCorrespondence}

1. Set $\sum_{n=1}^{N} \operatorname{card}\left(P_{n}\right)$ nodes with labels $(k, n)\left(k=1, \ldots, \operatorname{card}\left(P_{n}\right)\right)$ to be leaves of a graph $G$.

2. Let $P=\bigcup_{n=1}^{N} P_{n}, \hat{P}_{n}=P_{n}(n=1, \ldots, N)$, and $\tau=0$.

3. Increase the scale $\tau$ by $\Delta \tau$, which is a small value so that $\sqrt{2 \Delta \tau}$ is negligible compared with the space intervals of the points in $\bigcup_{n=1}^{N} \hat{P}_{n}$.

4. For each point $p_{i}$ in each point set $\hat{P}_{n}$, update the position of $p_{i}$ by maximizing $f(P ; \boldsymbol{x}, \tau)$ with $p_{i}$ as the initial point. If $p_{i}$ falls into a local maximum of another blob corresponding to a point $p_{j} \in \hat{P}_{m}$, remove $p_{i}$ from $\hat{P}_{n}$ and add a new node with two branches attached to the nodes labeled $(i, n)$ and $(j, m)$ in $G$. The new node inherits the label $(j, m)$.

5. If $\operatorname{card}\left(\hat{P}_{n}\right)=0$ for all but one of the point sets, then stop; otherwise go to Step 3.

The resulting graph $G$ is a disconnected graph consisting of subtrees of the scale-space tree of $\bigcup_{n=1}^{N} P_{n}$. Each subtree describes a point cluster composed of points among the point sets. The point correspondences are hierarchically indicated by the edges of the subtrees.

The hierarchical clustering continues until all but one point set are exterminated. In the early stages of the iteration, points of the same point set may compose a cluster. Since such a point set is locally denser than other point sets, it is feasible to determine the correspondence of a representative point of the cluster. The peak of the merged blobs in scale space plays the role of the representative point of the cluster. Algorithm II automatically selects the scales at which corresponding pairs are identified on the basis of the scale-space framework.

We execute the point correspondence algorithm for the pair of artifical point sets in $160 \times 160$ 2D space shown in Fig. 47(a). Each point set consists of 300 points, and the regions of the point 
sets partially overlap. Figure 47(b) shows the correspondences of points and clusters found using Algorithm PointCorrespondence. The hierarchy of the correspondences in the scale space is shown in Fig. 48. The iteration stopped at the scale where the clusters remain detached. We can see a cluster of points of the two point sets in the overlapping region, which is indicated by $\mathrm{C}$ in Fig. 48(a). The top node ( $\mathrm{V}_{\mathrm{C}}$ in Fig. 48(b)) of the subtree approximates the barycentre of this cluster, since the cluster points are dense enough to be isolated.

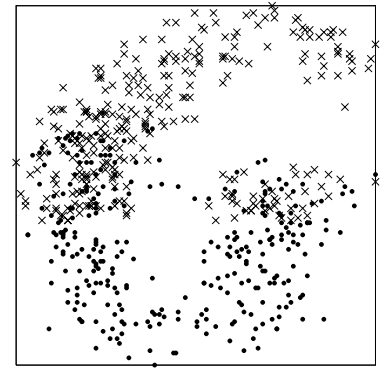

(a)

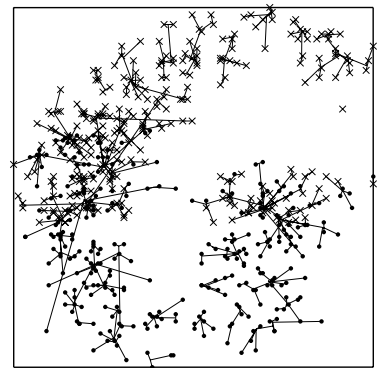

(b)

Figure 47: (a) Two point sets indicated by crosses and dots. (b) Correspondences of points and clusters.

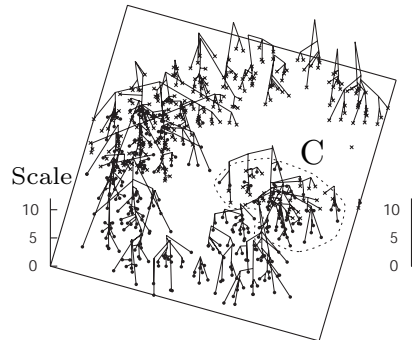

(a)

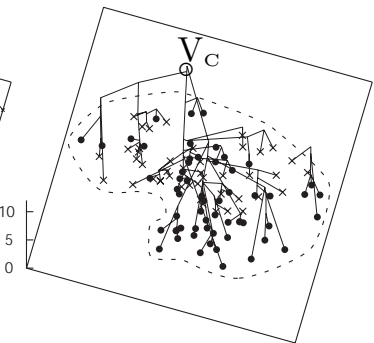

(b)

Figure 48: (a) Correspondences of points and clusters in scale space. (b) Magnified view of the cluster region $\mathrm{C}$ in (a).

\subsection{Discussion}

The main novelties of principal component analysis in the Gaussian scale space are as follows

- Observation of topological structure of point cloud in scale space and the reduction of dimensionality with respect to scale.

- Explanation scale-based clustering and cluster validity in terms of statistical significance.

- Derivation of stochastic moment generator from a PDF estimated in scale space.

- Scale-based PCA and a criterion for choosing principal components.

The topological structure of the point cloud density is naturally determined by the flow of probability density with respect to the scale. Since the bandwidth of the Gaussian kernel, i.e., scale, controls the information quantity, we can observe structural simplification of the estimated point cloud density with respect to the loss of information. We explained the validity of clusters discovered by the scale-based hierarchical clustering. The statistical significance of the reproducibility of the cluster discovery is guaranteed by the rejection method using the life of mode in scale space. The critical value of the scale in the rejection method is the so-called critical scale, which discriminates between valid and invalid clusters.

The scale-based PCA is mathematically derived from the first- and second-order stochastic moments calculated by the Gaussian kernel density estimate of a point cloud. The scale-based PCA can be applied to individual valid clusters. Equation (137) suggests that the bandwidth of the kernel contributes to the significance of eigenvalues if one switches the PDF model from parametric 
to nonparametric Consequently, the eigenvalues of principal components must be greater than the square of the critical scale. Otherwise, the validity of the cluster is not guaranteed in the subspace spanned by eigenvectors with the small eigenvalues.

The determination of the number of principal components is an essential problem in PCA, and it has been treated extensively in the literature prior to subspace methods. We refer to (Jackson, 1993) for a comprehensive overview. For this problem, we would like to note that the term $\sigma_{c}^{2} \boldsymbol{I}$ in Eq. (137) induces the so-called sphericity of the cluster. The sphericity is the degree of how spherical the distribution is. Generically, the covariance matrix of a point cloud or its cluster does not have a purely zero eigenvalue. That is, the estimated density distribution is not confined in a subspace of $\mathbb{R}^{d}$ but it is $d$-dimensional elliptic in a strict sense. Our scale-based PCA implies that the cluster requires some sphericity to be identified as a valid cluster, and the sphericity need to be greater than the critical scale in radius. From this point of view, only the principal components with eigenvalues greater than the critical scale might reflect a valid contribution to the similarity measure with subspace in classification methods.

Possible topics of future work for the results in this section will be as follows:

- Dependence of the critical scale on dimensionality and cardinality. As the cardinality increases, the point cloud provides the detailed structure of its density distribution, and the clusterability is enhanced. Thus, the critical scale will decrease with increasing cardinality.

- Relationship to the nearest-neighbour (NN) clustering of data points. It is known that the NN distance presents the Weibull distribution with the scale parameter as the density of data points and with shape parameter as the dimensionality. This will enable us to estimate the dimensionalities of clusters by parameter estimation.

\section{Digital Scale-Space Analysis}

This section focuses on the computation of stationary curves, which are sometimes called fingerprints for one-dimensional real signals in the linear scale space. Images for the analysis in the linear scale space are expressed as digital images for each quantised scale. Therefore, we develop a discrete version of the Gaussian scale-space analysis, which employs the results of digital image analysis. For the application of Gaussian scale space analysis to time-varying images and objects, our method has advantages because our method is based on the digital geometry on a plane which is suitable for the computation in digital computers.

We assume that the functions are defined on the one- and two-dimensional lattice points $\mathbb{Z}$, and $\mathbb{Z}^{2}$, respectively, and the scale parameter $\tau$ is nonzero, that is, $\tau \in \mathbb{Z}_{0}$, where $\mathbb{Z}$ and $\mathbb{Z}_{0}$ are the set of integers and the set of non-negative integers, respectively. We set $f_{n}(\tau)=f(n, \tau), f_{m}(\tau)=f(m \tau)$ and $f_{m n}(\tau)=f(m, n, \tau)$ for the one- and two-dimensional functions.

First, we deal with the digital diffusion for one-dimensional digital functions. The digital diffusion equation is defined as

$$
f_{m+1}(\tau+1)-f_{m}(\tau)=\alpha\left(f_{m+1}(\tau)-\frac{1}{2} f_{m}(\tau)+f_{m-1}(\tau)\right)
$$

with an appropriate boundary condition. For the classical Gaussian scale-space analysis, we assume that

$$
\lim _{|\boldsymbol{x}| \rightarrow \infty} \exp (\boldsymbol{x} \tau) f(\boldsymbol{x}, \tau)=0
$$

For discrete images, in this study, we assume the condition

$$
f(1)-f(0)=0, f(M+1)-f(M)=0
$$

for a sufficiently large positive integer $M$. With the boundary condition of Eq. (140), the linear diffusion equation is expressed as

$$
\boldsymbol{f}(\tau+1)-\boldsymbol{f}(\tau)=\alpha \frac{1}{2} \boldsymbol{C} \boldsymbol{f}(\tau), \quad \boldsymbol{C}=\left(\begin{array}{cccccc}
-1 & 1 & 0 & \cdots & 0 & 0 \\
1 & -2 & 1 & \cdots & 0 & 0 \\
\vdots & \vdots & \vdots & \ddots & \vdots & \vdots \\
0 & 0 & 0 & \cdots & 1 & -1
\end{array}\right)
$$


for $\boldsymbol{f}(\tau)=\left(f_{1}(\tau), f_{2}(\tau), \cdots, f_{M}(\tau)\right)^{\top}$. Setting $\boldsymbol{f}=\boldsymbol{f}(0)$, Eq. (141) is re-expressed as

$$
\boldsymbol{f}(\tau)=\boldsymbol{G}^{\tau}, \boldsymbol{G}=\boldsymbol{I}+\alpha \frac{1}{2} \boldsymbol{C} .
$$

The matrix $\boldsymbol{G}^{\tau}$ satisfies the relation ${ }^{5}$

$$
\boldsymbol{G}\left(\tau+\tau^{\prime}\right)=\boldsymbol{G}(\tau) \boldsymbol{G}\left(\tau^{\prime}\right), \boldsymbol{G}(0)=\boldsymbol{I}
$$

If we adopt

$$
f_{m+1}(\tau+1)-f_{m}(\tau)=\alpha\left(f_{m+1}(\tau+1)-\frac{1}{2} f_{m}(\tau+1)+f_{m-1}(\tau+)\right)
$$

we have the relation

$$
\boldsymbol{f}(\tau)=\boldsymbol{F}^{\tau}, \quad \boldsymbol{F}=\left(\boldsymbol{I}-\alpha \frac{1}{2} \boldsymbol{C}\right)^{-1}
$$

for $\left|1-\alpha \frac{1}{2}\right|<1$.

Therefore, Eq. (142) is a discrete version of the the Lie-group-based expression of the linear diffusion equation ${ }^{6}$.

The eigenvalues and eigenvectors of the matrix $\boldsymbol{C}$ are

$$
\begin{aligned}
\sigma_{k} & =2 \cos 2 \beta_{k}, \\
\boldsymbol{v}_{k}^{M} & =\left(\cos \beta_{k}, \cos 3 \beta_{k}, \cdots, \cos (2 M-1) \beta_{k}\right)^{\top},
\end{aligned}
$$

for $\beta_{k}=\frac{k \pi}{2 M}$. Therefore, the discrete Cosine transform matrix (DCT Matrix) (Strang and Nguyen, 1996; Demmel, 1997) $\boldsymbol{V}$, where

$$
\boldsymbol{V}=\left(\boldsymbol{v}_{0}, \boldsymbol{v}_{1} \cdots, \boldsymbol{v}_{M-1}\right),
$$

is the eigenmatrix of matrix $\boldsymbol{C}$ which orthogonalises matrix $\boldsymbol{C}$ as $\boldsymbol{C}=\boldsymbol{V} \boldsymbol{D} \boldsymbol{V}^{\top}$. Therefore, setting $f=f(0)$, the equation

$$
\boldsymbol{G} \boldsymbol{f}(\tau)=\boldsymbol{V} \boldsymbol{\Lambda}^{\tau} \boldsymbol{V}^{\top} \boldsymbol{f}, \boldsymbol{\Lambda}=\boldsymbol{I}+\frac{1}{2} \alpha \boldsymbol{D}
$$

computes $\boldsymbol{f}(\tau)$.

For two-dimensional digital images, the diffusion equations with the cyclic, the first type, and the second type boundary conditions, are expressed as

$$
\boldsymbol{f}(\tau+1)-\boldsymbol{f}(\tau)=\alpha^{2} \frac{1}{4}(\boldsymbol{C} \otimes \boldsymbol{C}) \boldsymbol{f}(\tau),
$$

where $\boldsymbol{A} \otimes \boldsymbol{B}$ is the Kronecker product ${ }^{7}$ of the two matrices $\boldsymbol{A}$ and $\boldsymbol{B}$.

\section{Summary and Conclusion}

The scale-space analysis is a basic approach to derive qualitative descriptions of an image. The hierarchical structure is wholly provided by the spatial gradient of the Gaussian scale-space image. The stationary curves and antidirectional figure-flow curves define the hierarchy of the local maxima, local minima, and saddle points of the image, which are regarded as the geometric feature points

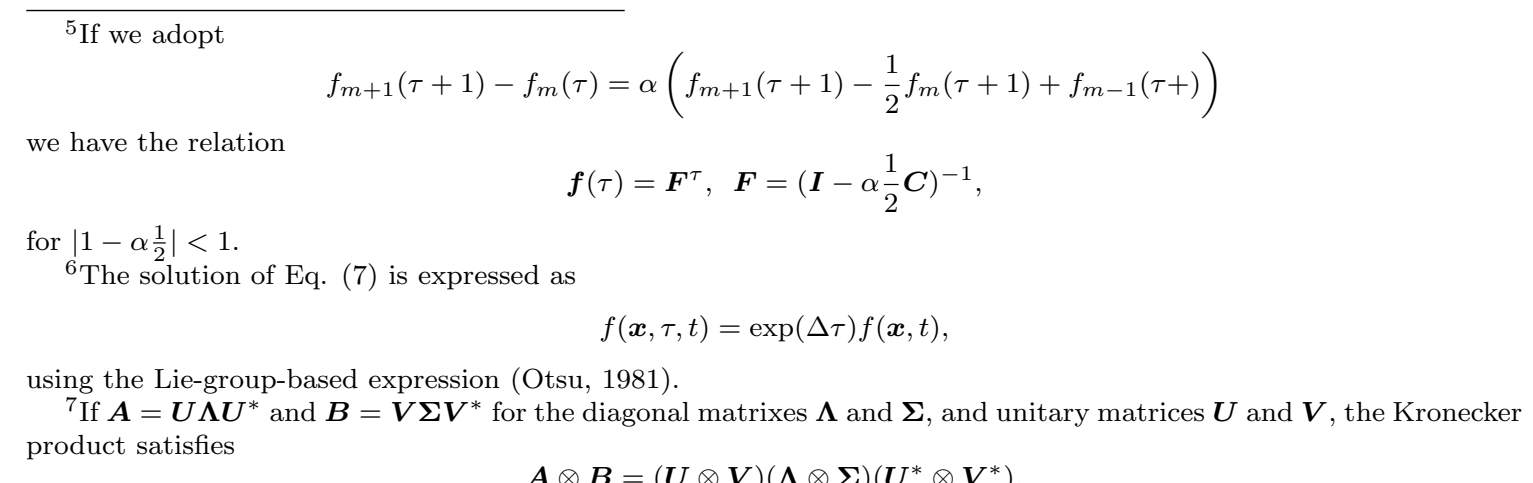


of the image corresponding to bright parts, dark cavities, and inbetween segments, respectively. A scale-space tree, which is constructed from the trajectory of singular points across scales in the Gaussian scale space, represents the hierarchical structure of the image.

The scale-space analysis of the image structure is simply extended to motion analysis. The apparent structure of objects in motion can be detected by observing a sequence of the scale-space trees which are derived from the sequence of images. It is possible to segment the image sequence into subsequences using the structural transitions detected by the scale-space trees.

Singular points in the Gaussian scale space provide fundamental features for the extraction of the dominant parts of an image. Employing the geometrical configuration of singular points, it is possible to construct a tree in scale space. This tree expresses a hierarchical structure of dominant parts. We clarified the graphical grammar for the construction of this tree in the linear scale space.

We also analysed the Canny operator using the Gaussian scale-space framework and found a theoretical strategy for the determination of parameters involved in Canny operation. Furthermore, we extracted the hierarchical relation of segments using the configuration of the saddle points in the Gaussian scale space. Moreover, we proposed a segmentation method and validation scheme for the segments in scale space to prevent under- and oversegmentation. Our segmentation method adopts the Canny edge detection as a geometric approach to boundary detection of the segments of a scale-space image.

We have attempted to make a first step towards a mathematical framework of principal component analysis on the basis of the scale-space theory on point cloud density and statistical principles of cluster discovery. We extended the Gaussian scale-space theory to the kernel density estimation of the point cloud in a Euclidean space of arbitrary dimension.

This research was supported by "Computational anatomy for computer-aided diagnosis and therapy: Frontiers of medical image sciences" funded by Grant-in-Aid for Scientific Research on Innovative Areas, MEXT, Japan, Grants-in-Aid for Scientific Research founded by Japan Society of the Promotion of Sciences and Grant-in-Aid for Young Scientists (A), NEXT, Japan.

\section{References}

J. Badaud, A. P. Witkin, M. Baudin, and R. Duda. Uniqueness of the gaussian kernel for scale space filtering. IEEE PAMI, 8(1):6-33, 1986.

J. W. Bruce and P. J. Giblin. Curves and Singularities, 2nd edition. Cambridge University Press, 1992.

J. Canny. A computational approach to edge detection. IEEE PAMI, 8(6):679-698, 1986.

M. A. Carreira-Perpinan and C. K. I. Williams. On the number of modes of a gaussian mixture. LNCS, 2695:625-640, 2003.

S. V. Chakravarthy and J. Ghosh. Scale-based clustering using the radial basis function network. IEEE Neural Networks, 7(5):1250-1261, 1996.

J. Chan, Y. Sato, and S. Tamura. Orientation space filtering for multiple orientation line segmentation. IEEE PAMI, 22(5):417-429, 2000.

J. Damon. Local morse theory for solutions to the heat equation and gaussian blurring. Journal of Differentia Equations, 115(2):368-401, 1995.

J. Damon. Generic properties of solutions to partial differential equations. Arch. Rational Mech. Anal., 140(4):353-403, 1997.

J. W. Demmel. Applied Numerical Linear Algebra. SIAM, 1997.

R. Duits, L. M. J. Florack, and B. M. ter Haar Romeny J. Graaf. On the axioms of scale space theory. Journal of Mathematical Imaging and Vision, 20(3):267-298, 2004.

E. Enomoto and T. Katayama. Structure lines of images. In Proc. of 3rd IJCAI, pages 811-815, 1976 .

H. Enomoto, N. Yonezaki, and Y. Watanabe. Picture Engineering, chapter H. Enomoto and N. Yonezaki and Y. Watanabe, pages 106-137. Springer, Berlin, 1982. 
L. M. J. Florack and A. Kuijper. The topological structure of scale-space images. Journal of Mathematical Imaging and Vision, 12(1):65-79, 2000.

L. D. Griffin and A. Colchester. Superficial and deep structure in linear diffusion scale space: Isophotes, critical points and separatrices. Image and Vision Computing, 13(7):543-557, 1995.

L. D. Griffin, A. C. F. Colchester, and G. P. Robinson. Scale and segmentation of grey-level images using maximum gradient paths. Image and Vision Computing, 10(6):389-402, 1992.

A. Hinneburg and D. A. Keim. An efficient approach to clustering in large multimedia databases with noise. In Proc. 4th International Conference on Knowledge Discovery and Data Mining, pages 58-65, 1998.

T. Iijima. Basic theory on the normalization of pattern (in case of typical one-dimensional pattern) (in Japanese). Bulletin of Electrotechnical Laboratory, 26:368-388, 1962.

T. Iijima. Basic theory on the normalization of two-dimensional visual pattern (in Japanese). Technical Report of IECE Japan, Information and Control, Pattern Recognition, pages 15-22, 1963.

T. Iijima. Basic equation of figure and observational transformations. Transaction of IECE Japan, C, 54-C:37-38, 1971.

T. Iijima. Pattern Recognition (in Japanese). Corona, Tokyo, 1973.

T. Iijima. The Fundamental Theory of Visual Information: The Foundation of the Pattern Recognition Problem. (in Japanese). Corona, Tokyo, 1999.

A. Imiya and K. Kawamoto. Learning dimensionality and orientations of 3D objects. Pattern Recognition Letters, 22(1):75-83, 2001.

A. Imiya, H. Ootani, and K. Kawamoto. Linear manifolds analysis: theory and algorithm. Neurocomputing, 57:171-187, 2004.

A. J. Izenman. Recent developments in nonparametric density estimation. Journal of the American Statistical Association, 86(413):205-244, 1991.

D. A. Jackson. Stopping rules in principal components analysis: A comparison of heuristic al and statistical approaches. Ecology, 74(8):2204-2214, 1993.

P. Johansen. On the classification of toppoints in scale space. Journal of Mathematical Imaging and Vision, 4(1):57-67, 1994.

P. Johansen, S. Skelboe, K. Grue, and J. D. Andersen. Representing signals by their toppoints in scale space. In Proc. of ICPR86, pages 215-217, 1986.

T. Kawashima, A. Imiya, and F. Nishida. Approximate tree distance (in Japanese). Technical Report of IEICE SIG PRMU, PRMU96:81-87, 1996.

J. J. Koenderink. The structure of images. Biological Cybernetics, 50(5):363-370, 1984.

R. Kothari and D. Pitts. On finding the number of clusters. Pattern Recognition Letters, 22(4): 405-416, 1999.

W. M. Krueger and K. Phillips. The geometry of differential operator with application to image processing. IEEE PAMI, 11(12):1254-1264, 1989.

A. Kuijper. The deep structure of Gaussian scale-space images. PhD thesis, Utrecht University, 2002 .

A. Kuijper and L. M. J. Florack. The hierarchical structure of images. IEEE Image Processing, 12 (9):1067-1079, 2003.

A. Kuijper and L. M. J. Florack. Using catastrophe theory to derive trees from images. Journal of Mathematical Imaging and Vision, 23(3):219-238, 2005. 
A. Kuijper, L. M. J. Florack, and M. A. Viergever. Scale space hierarchy. Journal of Mathematical Imaging and Vision, 18(2):169-189, 2003.

Y. Leung, J.-S. Zhang, and Z.-B. Xu. Clustering by scale-space filtering. IEEE PAMI, 22(12): 1396-1410, 2000.

L. M. Lifshitz and S. M. Pizer. A multiresolution hierarchical approach to image segmentation based on intensity extrema. IEEE PAMI, 12(6):529-540, 1990.

T. Lindeberg. Scale-Space Theory in Computer Vision. Kluwer, Boston, 1994.

T. Lindeberg. Feature detection with automatic selection. International Journal of Computer Vision, 30(2):79-116, 1998.

M. Lindenbaum, M. Fischer, and A. Bruckstein. On gabor's contribution to image enhancement. Pattern Recognition, 27(1):1-8, 1994.

M. Loog, J. J. Duistermaat, and L. M. J. Florack. On the behavior of spatial critical points under gaussian blurring. LNCS, 2106:183-192, 2001.

M. C. Minnotte and D. W. Scott. The mode tree: A tool for visualization of nonparametric density features. Journal of Computational and Graphical Statistics, 2(1):51-68, 1993.

E. Muybridge. Animal Locomotion. University of Pennsylvania, Philadelphia, 1887.

L. Najman and M. L. Schmitt. Watershed of a continuous function. Signal Processing, 38(1):99-112, 1994.

E. Nakamura and N. Kehtarnavaz. Determining number of clusters and prototype locations via multi-scale clustering. Pattern Recognition Letters, 19(14):1265-1283, 1998.

F. Nishida. Approximate tree distance. Bacheler Theses, Department of Information Sciences, Chiba University, 1994.

O. F. Olsen and M. Nielsen. Generic events for the gradient squared with application to multi-scale segmentation. LNCS, 1251:101-112, 1997.

N. Otsu. Mathematical studies on feature extraction in pattern recognition (in Japanese). Researches of The Electrotechnical Laboratory, 818, 1981.

A. Papoulis. The Fourier Integral and its Applications. McGraw-Hill, 1962.

A. Papoulis. Probability, Random Variables, and Stochastic Processe. McGraw-Hill, 1965.

E. Parzen. On estimation of a probability density function and mode. The Annals of Mathematical Statistics, 33(3):1065-1076, 1962.

M. Pelillo, K. Siddiqi, and S. W. Zucker. Matching hierarchical structures using association graphs. IEEE PAMI, 21(11):1105-112-, 1999.

S. J. Roberts. Parametric and non-parametric unsupervised cluster analysis. Pattern Recognition, 30(2):261-272, 1997.

Ian R. Rorteous. Geometric Differentiation 2nd edition. Cambridge University Press, 2001.

M. Rosenblatt. Remarks on some nonparametric estimates of a density function. The Annals of Mathematical Statistics, 27(3):832-837, 1956.

T. Sakai and A. Imiya. Figure field analysis of linear scale-space image. LNCS, 3459:374-385, 2005a.

T. Sakai and A. Imiya. Scale-space hierarchy of singularities. LNCS, 3753:181-192, 2005b.

T. Sakai and A. Imiya. Gradient structure of image in scale space. Journal of Mathematical Imaging and Vision, 28(3):243-257, 2007.

T. Sakai, A. Imiya, T. Komazaki, and S. Hama. Critical scale for unsupervised cluster discovery. LNCS, 4571:218-232, 2007. 
Y. Sato, S. Nakajima, N. Shiraga, H. Atsumi, S. Yoshida, T. Koller, G. Gerig, and R. Kikinis. Threedimensional multi-scale line filter for segmentation and visualization of curvilinear structures in medical images. Medical Image Analysis, 2(2):143-168, 1998.

A. Simmons, S. R. Arridge, P. S. Tofts, and G. J. Barker. Application of the extremum stack to neurological mri. IEEE Medical Imaging, 17(3):371-382, 1998.

J. Sporring and J. Weickert. Information measures in scale-spaces. IEEE Information Theory, 45 (3):1051-1058, 1999.

G. Strang and T. Nguyen. Wavelets and Filter Banks. Wellesley-Cambridge Press, 1996.

H. Theisel, T. Weinkauf, H. C. Hege, and H.-P. Seidel. Saddle connectors - an approach to visualizing the topological skeleton of complex 3d vector fields. In Proc. of IEEE Visualization 2003, pages 225-232, 1986.

UMass. Box. http://vis-www.cs.umass.edu/ vislib/Motion/directory.html, 2010.

J. Weickert, S. Ishikawa, and A. Imiya. Gaussian Scale-Space Theory, chapter Ch. 4, On the history of Gaussian scale-space axiomatics, pages 45-59. Kluwer Academic Publisher, Dordrecht, 1997.

J. Weickert, S. Ishikawa, and A. Imiya. Linear scale-space has first been proposed in Japan. Journal of Mathematical Imaging and Vision, 10(3):237-255, 1999.

A. P. Witkin. Scale space filtering. In Proc. of 8th IJCAI, pages 1019-1022, 1983.

A. L. Yuille and T. A. Poggio. Scale space theory for zero crossings. IEEE PAMI, 8(1):15-25, 1986.

N. Y. Zhao and T. Iijima. Theory on the method of determination of view-point and field of vision during observation and measurement of figure (in Japanese). Transaction of IECE, Japan D, J68-D:508-514, 1985a.

N.-Y. Zhao and T. Iijima. A theory of feature extraction by the tree of stable view-points (in Japanese). Transaction of IECE, Japan D, J68-D:1125-1135, 1985 b. 